\begin{tabular}{|c|l|}
\hline Title & Viscosity solutions with shocks \\
\hline Author(s) & Giga, Y. \\
\hline Citation & Hokkaido University Preprint Series in Mathematics, 519, 1-58 \\
\hline Issue Date & 2001-02 \\
\hline DOI & 10.14943/83665 \\
\hline Doc URL & http://hdl.handle.net/2115/69269 \\
\hline Type & bulletin (article) \\
\hline File Information & pre519.pdf \\
\hline
\end{tabular}

Instructions for use 
Viscosity solutions with shocks

Yoshikazu Giga

Series \#519. February 2001 


\section{HOKKAIDO UNIVERSITY PREPRINT SERIES IN MATHEMATICS}

\#494 T. Mikami, Optimal control for absolutely continuous stochastic processes and the mass transportation problem, 17 pages. 2000.

\#495 M. Arisawa and Y. Giga, Anisotropic curvature flow in a very thin domain, 21 pages. 2000.

\#496 T. Nakazi, Backward shift invariant subspaces in the bidisc, 9 pages. 2000.

\#497 Y. Giga, K. Inui, J. Kato and S. Matsui, Remarks on the uniqueness of bounded solutions of the NavierStokes equations, 4 pages. 2000.

\#498 Y. Giga, M. Paolini and P. Rybka, On the motion by singular interfacial energy, 21 pages. 2000.

\#499 J. Escher, Y. Giga and K. Ito, On a limiting motion and self-interactions of curves moved by the intermediate surface diffusion flow, 12 pages. 2000.

\#500 I. Tsuda and S. Kuroda, Cantor coding in the hippocampus, 20 pages. 2000.

\#501 M. Tsujii, Fat solenoidal attractors, 20 pages. 2000.

\#502 A. Arai, Ground state of the massless Nelson model without infrared cutoff in a non-Fock representation, 19 pages. 2000.

\#503 Y. Giga, S. Matsui and O. Sawada, Global existence of two-dimensional Navier-Stokes flow with nondecaying initial velocity, 19 pages. 2000.

\#504 A. Inoue and Y. Kasahara, Partial autocorrelation functions of the fractional ARIMA processes with negative degree of differencing, 14 pages. 2000.

\#505 T. Nakazi, Interpolation problem for $\ell^{1}$ and a uniform algebra, 12 pages. 2000.

\#506 R. Kobayashi and Y. Giga, On anisotropy and curvature effects for growing crystals, 38 pages. 2000.

\#507 A. Arai, Instability in the spectral and the Fredholm properties of an infinite dimensional Dirac operator on the abstract Boson-Fermion Fock space, 6 pages. 2000.

\#508 A. Arai, Supersymmetric methods for constructing soliton-type solutions to multi-component nonlinear Schrödinger and Klein-Gordon equations, 22 pages. 2000.

\#509 Y. Tonegawa, Phase field model with a variable chemical potential, 30 pages. 2000.

\#510 Y. Giga, Shocks and very strong vertical diffusion, 11 pages. 2000.

\#511 S. Izumiya \& N. Takeuchi, Special curves and raled surfaces, 18 pages. 2001.

\#512 S. Izumiya, Generating families of developable surfaces in $R^{3}, 18$ pages. 2001.

\#513 S. Izumiya, K. Maruyama, Transversal topology and singularities of Haefliger foliations, 8 pages. 2001.

\#514 S. Izumiya, D-H. Pei \& T. Sano, Singularities of hyperbolic Gauss maps, 27 pages. 2001.

\#515 S. Izumiya, N. Takeuchi, Generic special curves, 12 pages. 2001.

\#516 S. Izumiya. D-H. Pei \& T. Sano, Horospherical surfaces of curves in hyperbolic space, 9 pages. 2001.

\#517 R. Yoneda, The composition operators on weighted bloch space, 8 pages. 2001.

\#518 M. Jinzenji, T. Sasaki, $N=4$ supersymmetric Yang-Mills theory on orbifold- $T^{4} / \mathbf{Z}_{2}, 18$ pages. 2001. 


\title{
Viscosity solutions with shocks
}

\author{
YOSHIKAZU GIGA \\ Hokkaido University
}

\begin{abstract}
A solution of single nonlinear first order equations may develop jump discontinuities even if initial data is smooth. Typical examples include a crude model equation describing some bunching phenomena observed in epitaxial growth of crystals as well as conservation laws where jump discontinuities are called shocks. Conventional theory of viscosity solutions does not apply.

We introduce a notion of proper (viscosity) solutions to track whole evolutions for such equations in multi-dimensional spaces. We establish several versions of comparison principles. We also study the vanishing viscosity method to construct a unique global proper solution at least when the evolution is monotone in time or the initial data is monotone in some sense under additional technical assumptions.

In fact, we prove that the graph of approximate solutions converges to that of a proper solution in the Hausdorff distance topology. Such a convergence is also established for conservation laws with monotone data. In particular, local uniform convergence outside shocks is proved.
\end{abstract}

\section{Introduction}

We are concerned with the Cauchy problem for a single nonlinear first order equation of the form

$$
\frac{\partial u}{\partial t}+H\left(u, \frac{\partial u}{\partial x_{1}}, \ldots, \frac{\partial u}{\partial x_{n}}\right)=0 \text { in } \mathbf{R}^{n} \times(0, \infty)
$$

$$
\left.u\right|_{t=0}=u_{0} \quad \text { in } \mathbf{R}^{n}
$$

whose solution $u=u\left(x_{1}, \ldots, x_{n}, t\right)$ may develop jump discontinuities in finite time even if the initial data $u_{0}$ is smooth. Our goal is to find a suitable notion of weak 
solutions so that the problem (1.1)-(1.2) is uniquely solvable beyond jump discontinuities. Even if we assume that

$$
H(r, 0, \ldots, 0)=0
$$

so far such a theory has been established only when $H$ has a divergence structure so that (1.1) is a conservation law of the form

$$
\frac{\partial u}{\partial t}+\sum_{i=1}^{n} \frac{\partial}{\partial x_{i}} f_{i}(u)=0 \quad \text { in } \mathbf{R}^{n} \times(0, \infty)
$$

where $f_{i}$ is a given function for $i=1, \ldots, n$. There is a notion of the entropy solution (which is a special distributional weak solution) so that (1.4) with (1.2) is uniquely solvable for any bounded initial data $u_{0}$ at least when $f_{i}$ 's are $C^{1}$. Moreover, the entropy solution is obtained by $L^{1}$-limit of solutions $u^{\varepsilon}$ of a regularized equation

$$
\frac{\partial u}{\partial t}+\sum_{i=1}^{n} \frac{\partial}{\partial x_{i}} f_{i}(u)=\varepsilon \Delta u \quad \text { in } \mathbf{R}^{n} \times(0, \infty)
$$

with (1.2) as $\varepsilon \rightarrow 0$. The construction of the entropy solution using (1.5) is often called a vanishing viscosity method since $\varepsilon$ represents a viscosity coefficient in some problems in fluid mechanics. The reader is referred to a recent nice book by C. Dafermos [12] for these results as well as the theory of entropy solutions of conservation laws. However, there are several important examples of (1.1) which is not of the form (1.4). We give here two typical examples:

$$
\begin{aligned}
& \frac{\partial u}{\partial t}-a\left(u,-\frac{\nabla u}{|\nabla u|}\right)|\nabla u|=0 \\
& \frac{\partial u}{\partial t}-b(u)\left(1+|\nabla u|^{2}\right)^{1 / 2}=0
\end{aligned}
$$

where $\nabla u=\left(\frac{\partial u}{\partial x_{1}}, \ldots, \frac{\partial u}{\partial x_{n}}\right)$ and $a$ and $b$ are given functions. Here (1.6) fulfills (1.3) while (1.7) does not unless $b$ is identically equals to zero. The equation (1.6) is a general form of homogeneous equations in the sense that $p \mapsto H(r, p)$ is positively homogeneous of degree one so it includes (1.4) as a special example. A typical interesting situation we consider is that $a(r, \cdot)$ and $b(r)$ are not nonincreasing as a function of $r$ and positive. (Under such assumptions (1.6) is no longer a conservation law.) As for conservation laws by the method of characteristics it is not difficult to see that the solution may develop jump discontinuities in finite time even if the 
initial data is smooth for (1.6) and (1.7) under these assumptions. We postpone to explain geometric and physical meaning of these equations later in this introduction.

For non divergence $H$ the theory of viscosity solutions initiated by M. Crandall and P.-L. Lions [11] has been developed for many years. The notion of the viscosity solution provides a unique global-in-time continuous (not necessarily $C^{1}$ ) solution for continuous initial data. For example if the initial data $u_{0}$ is continuous and periodic in $x_{i}$ 's then there exists a unique global continuous viscosity solution $u$ of (1.1)-(1.2) provided that

$$
r \mapsto H(r, p) \text { is nondecreasing for each } p \in \mathbf{R}^{n}
$$

and that $H$ is continuous. Moreover, the viscosity solution $u$ is obtained by locally uniform limit (in $\mathbf{R}^{n} \times[0, \infty)$ ) of solution $u^{\varepsilon}$ of a regularized problem

$$
\frac{\partial u}{\partial t}+H(u, \nabla u)=\varepsilon \Delta u \text { in } \mathbf{R}^{n} \times(0, \infty)
$$

with (1.2) as $\varepsilon \rightarrow 0$. The reader is referred to several nice books [35], [4], [3] and a survey article [10] for these results. The equations (1.6) and (1.7) do not fulfill the monotonicity condition (1.8) (or its weaker variants) under our assumptions and solutions may develop jump discontinuities. So the conventional theory of viscosity solutions should not apply to construct a global solution with jump discontinuities.

In this paper we introduce a new notion of solutions (which we call proper viscosity solutions) which is actually stronger than that of conventional viscosity solutions for discontinuous functions to solve (1.1), (1.2) including (1.4), (1.6), (1.7) without assuming the monotonicity (1.8). We expect that the limit of solutions of a regularized problem (1.9) as $\varepsilon \rightarrow 0$ is characterized by our proper viscosity solution. In this paper as the first attempt we prove that the limit of solutions of (1.9), (1.2) converges to the proper viscosity solution of $(1.1),(1.2)$ when the evolution is monotone in time or initial data fulfills some monotonicity condition. Before explaining the new notion we give typical convergence results on (1.9) where the limit is a proper viscosity solution. To avoid technical complexity we first impose a periodic boundary condition on (1.9) and (1.1). In other words we consider $u$ as a function on $\mathbf{T}^{n} \times(0, \infty)$, where $\mathbf{T}^{n}=\prod_{i=1}^{n}\left(\mathbf{R} / \omega_{i} \mathbf{Z}\right)$ with some $\omega_{i}>0, i=1, \ldots, n$. For simplicity of presentation in the introduction we shall assume that the initial data. $u_{0}$ is continuous i.e., $u_{0} \in C\left(\mathbf{T}^{n}\right)$ although our theory allows discontinuous initial data. We begin with typical results when the evolution is monotone in time for (1.6) and (1.7). 
Theorem 1.1. Assume that $H$ is locally Lipschitz (continuous) and that (i) $H(r, p)=-a(r,-p /|p|)|p|$ or (ii) $H(r, p)=-b(r)\left(1+|p|^{2}\right)^{1 / 2}$. Let $u^{\varepsilon}$ be the solution of (1.9) with $\left.u^{\varepsilon}\right|_{t=0}=u_{0} \in C\left(\mathbf{T}^{n}\right)$. Assume that inf $a>0$ and inf $b>0$. In the case of (i) assume further more that $u_{0}$ fulfills 'no plateau condition' in the sense that the closure of a super level set $\left\{u_{0}>c\right\}$ equals $\left\{u_{0} \geq c\right\}$ for all $c$ except $c=\max u_{0}$ or min $u_{0}$. In the case of (ii) assume furthermore that

$$
\int_{1}^{\infty} b(r)^{-1} d r=\infty \text { and } \int_{-\infty}^{-1} b(r)^{-1} d r=\infty
$$

Then

$$
\operatorname{sg} u^{\varepsilon} \rightarrow \operatorname{sg} u \quad \text { as } \varepsilon \rightarrow 0
$$

for some upper semicontinuous function $u$ in the sense of convergence of closed sets, or equivalently in the sense of the Hausdorff distance topology in (the class of all closed subsets with compact boundary of) $\mathbf{T}^{n} \times \mathbf{R} \times[0, T]$ for any $T>0$.

Here sg $u^{\varepsilon}$ denote the subgraph set of $u^{\varepsilon}$ defined by

$$
\operatorname{sg} u^{\varepsilon}=\left\{(x, y, t) \in \mathbf{T}^{n} \times \mathbf{R} \times[0, \infty) ; \quad y \leq u^{\varepsilon}(x, t)\right\}
$$

Note that no monotonicity assumption like (1.8) is imposed. The assumption (1.10) is equivalent to say that spatially constant solutions do not blow up in finite time. If (1.3) is fulfilled such an assumption is of course unnecessary. The no plateau assumption is imposed for technical reasons of the proof. Our theory applies to a more general class of the equations including (1.6) and (1.7). Unfortunately, our assumption inf $a>0$ does not allow to apply Theorem 1.1 to the conservation law (1.4). We give another convergence result for homogeneous equation (1.6) including (1.4) for strictly spokewise decreasing initial data $u_{0}$. A precise definition of this monotonicity condition will be given in $\S 5.3$. It at least requires that $s \mapsto u_{0}\left(s x_{0}\right)$, $\left(x_{0} \in \mathbf{R}^{n}, x_{0} \neq 0\right)$ is decreasing as a function of $s$.

Theorem 1.2. Assume that $H(r, p)=-a(r,-p /|p|)|p|$ is locally Lipschitz. Let $u^{\varepsilon}$ be the solution of (1.9) with $\left.u^{\varepsilon}\right|_{t=0}=u_{0} \in C\left(\mathbf{R}^{n}\right)$. Assume that $u_{0}$ is strictly spokewise decreasing and that $u_{0}$ is (locally) constant and continuous outside some ball. Then (HC) holds with $\mathrm{T}^{n}$ replaced by every closed ball $B_{R}(0)$ of radius $R$ centered at $0 \in \mathbf{R}^{n}$. Moreover, $u(\cdot, t)$ is strictly spokewise decreasing in $\mathbf{R}^{n}$ and for each $T>0 u$ is (locally) constant and continuous outside $B_{R_{0}}(0) \times[0, T)$ for some $R_{0}>0$. 
When $n=1$, our assumption on $u_{0}$ allows monotone initial data which is strictly monotone outside the place where $u_{0}=\max u_{0}$ or min $u_{0}$. The limit $u$ is characterized as a proper viscosity solution.

Theorem 1.3. Let $u$ be as in Theorem 1.1. or Theorem 1.2. Then $u$ is the unique proper viscosity solution of (1.1), (1.2) with corresponding $H$.

Since (1.9) is uniquely solvable when $H(r, p)$ is Lipschitz in $p \in \mathbf{R}^{n}$ uniformly in $r \in[-R, R]$ and $\left|H(r, p)-H\left(r^{\prime}, p\right)\right| /\left|r-r^{\prime}\right|(1+|p|)$ is bounded on $[-R, R] \times \mathbf{R}^{n}$ for every $R>0$, this result yields unique global existence of a proper viscosity solution for (1.6) and (1.7) at least when $a$ and $b$ are $C^{1}$. The convergence (HC) gives the local uniform convergence off jump discontinuities of $u$. Let $\Sigma$ be the closure in $\mathbf{R}^{n} \times[0, \infty)$ of the set of all points at which $u$ is discontinuous.

Corollary 1.4. The convergence $u^{\varepsilon} \rightarrow u$ is locally uniform in $\mathbf{R}^{n} \times[0, \infty) \backslash \Sigma$.

Results in Theorem 1.2 apply to the conservation law (1.4) if $f_{i} \in C^{2}(\mathbf{R})$ for $i=$ $1, \ldots, n$. As anticipated the limit proper solution is an entropy solution.

Corollary 1.5. Assume that $f_{i} \in C^{2}(\mathbf{R})$ for $i=1, \ldots, n$. Let $u^{\varepsilon}$ be the solution of (1.5) with $\left.u^{\varepsilon}\right|_{t=0}=u_{0} \in C\left(\mathbf{R}^{n}\right)$. Assume that $u_{0}$ is strictly spokewise decreasing and locally constant and continuous outside some ball. Then $\mathrm{sg} u^{\varepsilon} \rightarrow \mathrm{sg} u$ for some upper semicontinuous function $u$ in the sense of the Hausdorff distance topology in $B_{R}(0) \times \mathbf{R} \times[0, T]$ for any $R>0, T>0$. Moreover, $u$ is the entropy solution of (1.1), (1.2).

It is of course likely that the monotonicity assumption on initial data of Corollary 1.5 is unnecessary. However, it seems that further development of the theory is necessary. We do not pursue this problem in the present paper since the paper is already long.

Our convergence results (Theorems 1.1, 1.2, Corollary 1.5) are new. Even for the conservation law, the subgraph convergence result in Corollary 1.6 is new although $L^{1}$-convergence of $u^{\varepsilon}$ is known for bounded initial data.

Convergence result like Corollary 1.4 is so far known only for (1.5) (under the assumption of genuine nonlinearity) when the space dimension $n=1$ as proved in [29], [41], where systems of conservation laws as well as convergence rate have been studied by matched asymptotic method. A stronger version of Theorems allowing discontinuous initial data shall be proved in $\S 6$ for more general $H$. We note that 
convergence of the graph of $u^{\varepsilon}$ can be also proved instead of the subgraphs (Remark $6.8)$.

Let us now explain the notion of a proper viscosity solution by using (1.6) when $n=1$. A proper viscosity solution is a proper viscosity subsolution and simultaneously a proper viscosity supersolution. As usual we only explain proper viscosity subsolutions since the definition for supersolution is symmetric. Roughly speaking, a proper viscosity subsolution $u$ of (1.6) is a usual viscosity subsolution the speed of whose jump discontinuities is controlled by "Rankine-Hugoniot" condition (cf. [12]). More precisely for $\left(x_{0}, t_{0}\right) \in \mathbf{R} \times(0, \infty)$ let $x=x(t)$ be a smooth curve through $\left(x_{0}, t_{0}\right)$ such that $u(x, t) \leq \mu$ for $x>x(t)$ near $\left(x_{0}, t_{0}\right)$ for some $\mu\left(<u\left(x_{0}, t_{0}\right)\right)$ independent of $(x, t)$. Then we require that an upper bound of the velocity $\dot{x}\left(t_{0}\right)$ of the 'shock':

$$
\dot{x}\left(t_{0}\right) \leq \alpha\left(u\left(x_{0}, t_{0}\right)\right), \quad-\alpha=\left(g_{I}\right)^{\prime}, g(r)=-\int a(r, 1) d r
$$

where $g_{I}$ denotes the convexification of $g$ on $I=\left[\mu, u\left(x_{0}, t_{0}\right)\right]$. Once such a bound holds for every such a curve $x(t)$ with $\mu$ and $\left(x_{0}, t_{0}\right)$, an upper semicontinuous viscosity solution $u$ is called a proper viscosity subsolution. It is not difficult to see that the speed bound is nothing but the speed calculated by the Rankine-Hugoniot condition for shocks jumping from $\mu$ to $u\left(x_{0}, t_{0}\right)$ when $a=a(r, p)$ is increasing in $r$. To define such a notion for general $n$ we need to replace $\dot{x}\left(t_{0}\right)$ by the normal velocity of an evolving surfaces instead of the curve $x=x(t)$. The bound is replaced by $-H^{I}\left(u\left(x_{0}, t_{0}\right),-\mathbf{n}\right)$ where $\mathbf{n}$ is the normal (pointing to the region $\{u \leq \mu\})$ of the surface at $\left(x_{0}, t_{0}\right)$ and $H^{I}(r,-\mathbf{n})=\left(g_{I}\right)^{\prime}$ with $g(r)=\int H(r,-\mathbf{n}) d r$ when $H(r, p)=-a(r,-p /|p|)|p|$. When we need to handle $H$ which is not positively homogeneous of degree one, we have to consider the recession function $H_{\infty}$ in $p$ instead of $H$ itself to define the bound for the speed; for example for (1.7) $H_{\infty}(r, p)=-b(r)|p|$. Fortunately, such technical difficulties are overcame and the notion of proper viscosity solutions is defined for (1.1) (allowing $(x, t)$-dependence in $H$ ) including (1.4), (1.6), (1.7). Moreover, from a variant of Corollary 1.5 (Corollary 7.5), a non admissible shock solution for (1.4) (two-valued weak solution satisfying the Rankine-Hugoniot condition but is not an entropy solution) is not a proper solution at least when $n=1$. (One is able to check this fact directly by definition; see Examples in $§ 2$.)

The proof of Theorems 1.1-1.3 consists of two ingradients : (i) stability of approximate solutions $u^{\varepsilon}$ of (1.9) and (ii) a kind of comparison principle for proper solutions. Both steps need several new ideas. For (i) we shall prove that the relaxed 
limit $\bar{u}=\limsup _{\varepsilon \rightarrow 0} u^{\varepsilon}$ and $\underline{u}=\liminf _{\varepsilon \rightarrow 0} u^{\varepsilon}$ (see $\S 6$ for definition) are proper viscosity sub- and supersolutions, respectively and that $\left.\bar{u}\right|_{t=0} \leq u_{0} \leq\left.\underline{u}\right|_{t=0}$. The proof that $\bar{u}$ (resp. $\underline{u}$ ) is a proper vicosity subsolution (supersolution) is not standard since test functions for proper viscosity subsolutions and usual subsolution for (1.9) are quite different. We construct a family of test functions $\varphi_{\varepsilon}$ for $u^{\varepsilon}$ converging to a function with jumps such that the speed of its level sets in the sharp layer approximates the upper bound $\alpha$ of the velocity of (1.11). This is a kind of a method of 'perturbed test functions' first proposed for homogenization problems in [13]. However, our construction is totally different. Our function $\varphi_{\varepsilon}$ is regarded as a viscous shock. The proof for $\left.\bar{u}\right|_{t=0} \leq u_{0} \leq\left.\underline{u}\right|_{t=0}$ is standard and it says that there is no initial layer.

For (ii) we establish several comparison principles. A conventional comparison principle (often called a strong comparison principle) for discontinuous viscosity solutions says that $v^{*} \leq w_{*}$ if $\left.v^{*}\right|_{t=0} \leq\left. w_{*}\right|_{t=0}$ for viscosity subsolution $v$ and supersolution $w[30]$, where $*$ denotes upper and lower semicontinuous envelope (see $\S 2$ for definition). Such a principle does not hold in our setting since otherwise all solution $u$ starting with $u_{0} \in C\left(\mathbf{T}^{n}\right)$ would satisfy $u^{*} \leq u_{*}$ yielding the continuity of $u$, which is not expected to our problem. Instead, for periodic case we establish a weak comparison principle saying that $\left.v^{*}\right|_{t=0}<\left.w_{*}\right|_{t=0}$ implies $v^{*}<w_{*}$ for a proper viscosity subsolution $v$ and a supersolution $w$. We study a kind of indicator functions of subgraphs of $v$ and $w$ for the proof. Unfortunately, such a weak comparison principle is too weak to guarantee the uniqueness of proper solutions. However, this is not a defect of our method. As pointed out by [5] for $\frac{\partial u}{\partial t}+(x-t)\left|\frac{\partial u}{\partial x}\right|=0$ viscosity solutions may not be unique for semicontinuous initial data. (It turns out that their solution is also our proper viscosity solution.) Although in this example $H$ depends also on $x$ and $t$, it suggests non uniqueness of proper viscosity solutions for some situations. The phenomena is related to fattening in the level set method $[37],[8],[14]$ for curvature flow problems as noted in [14]; see also [38] and a review article [23]. One way to get uniqueness is to impose that the sign of $a$ and $b$ for (1.6) and (1.7) is definite so that evolution is monotone. ('No plateau condition' is also invoked for (1.6).) Another way is to impose strictly spokewise monotonicity on initial data for (1.6). This corresponds to star-sharpedness in the curvature flow problem [38]. These assumptions turn to remove the possibility of non uniqueness. For example, for (1.6) and (1.7) we establish a comparison principle (which we call a proper comparison principle) saying that $\left.v^{*}\right|_{t=0} \leq\left.\left(w_{*}\right)^{*}\right|_{t=0}$ implies $v^{*} \leq\left(w_{*}\right)^{*}$ under suitable assumptions on $a, b$ and initial data. The proper comparison principle with stability yields $\bar{u}=(\underline{u})^{*}$ (cf. Theorem 6.11). This implies (HC). It also implies that 
the limit $\bar{u}$ is the unique proper viscosity solution. In the case that a proper solution is not expected to be unique, the convergence result may not be true. However, one might expect that Perron's method [30] yields global existence of maximal or minimal solutions. Moreover, such solutions are expected to be characterized by a level set approach as developed in [26], [27] for (1.1) with (1.8) when the initial data is discontinuous. We do not pursue these directions in the present paper. Under the assumption (1.8) or its variant there are several other works on (1.1) with discontinuous initial data (e.g. [6], [7]) when $H$ is assumed to be convex or concave in $\nabla u$. We emphasize here that our theory does not require any monotonicity condition like (1.8).

Here is an idea to prove the proper comparison principle. When inf $b>0$, the proper comparison principle for (1.7) is established by applying the weak comparison principle for $v$ and $w_{\delta}$ to get $v^{*}<w_{\delta *}$, where $w_{\delta}(x, t)=w(x, t+\delta)$ and letting $\delta \rightarrow 0$. The proof for (1.6) under inf $a>0$ is similar. However, we are forced to invoke 'no plateau condition' since strictly monotonicity of solution holds only for its level sets. We do not explain the details in the introduction. For strictly spokewise decreasing initial data we rescale $w$ by $w^{\lambda}(x, t)=w(\lambda x, \lambda t)$ and apply a kind of weak comparison principle for $v$ and $w^{\lambda}$ for $\lambda \in(0,1)$. Letting $\lambda \uparrow 1$ yields $v^{*} \leq\left(w_{*}\right)^{*}$ to get the proper comparison principle. Here we invoke the property that (1.6) is invariant under the rescaling $w^{\lambda}$.

With aid of the theory of conservation laws we prove that $\bar{u}=\underline{u}$ almost everywhere for (1.4), (1.5) and it agrees with the entropy solution (without assuming monotonicity assumptions on $u_{0}$ ). It turns out that the entropy solution equals a proper solution almost everywhere and any proper solution of (1.4) with (1.2) agrees with the entropy solution almost everywhere (Remark 7.4). In general it is possible to prove that sg $\bar{u} \backslash \mathrm{sg}(\underline{u})^{*}$ has zero Lebesgue measure; however, it is not clear that $\bar{u}=(\underline{u})^{*}$ unless $u_{0}$ is strictly spokewise decreasing or increasing (cf. $\S 7$ ).

We remark that our problem relates to interface equations with singular interfacial energy. We explain its typical example of the form

$$
V=-\operatorname{div}_{\Gamma_{t}}(\nabla \gamma)(\mathbf{n}) \text { on } \Gamma_{t}
$$

Here $\Gamma_{t}$ denotes an evolving hypersurface in $\mathbf{R}^{d}$ with normal $\mathbf{n}$ and the normal velocity $V$. The function $\gamma: \mathbf{R}^{d} \rightarrow[0, \infty)$ is positively homogeneous of degree one and positive outside the origin. We assume that $\gamma$ is convex and call interfacial energy density. If $\gamma(p)=|p|,(1.12)$ is the mean curvature flow equation. If $\gamma$ may not be $C^{1}$, the regularizing effect in (1.12) turns to be nonlocal. Such problems are 
first proposed by J. Taylor [40] and independently by S. Angenent and M. Gurtin [2] especially when $\gamma$ is crystalline (i.e. piecewise linear). In the case $n=2$ the problem in well-studied. A typical feature is that the regularizing (diffusion) effect is very strong so that it is nonlocal and forms flat portions called facets. The reader is referred to [15], [17], [18], [19], [20] and [25] for the state of arts. For $n=3$ see [25] and papers cited there. As noted in [16] if the equation is spatially inhomogeneous, for example,

$$
V=b(y)-\operatorname{div}_{\Gamma_{t}}(\nabla \gamma)(\mathbf{n}) \text { on } \Gamma_{t} \subset \mathbf{R}_{x}^{n} \times \mathbf{R}_{y}=\mathbf{R}^{d}
$$

a little is known for well-posedness. In particular, we do not have the notion of solutions so that the problem is locally solvable for general surface $\Gamma_{t}$. We remark that the graph $\Gamma_{t}$ in $\mathbf{R}^{n+1}$ of our proper viscosity solution of (1.7) is regarded a kind of solution of (1.13) with $\gamma(p)=M\left|p_{n+1}\right|, p=\left(p_{1}, \ldots, p_{n}, p_{n+1}\right)$ for sufficiently large $M$. A very strong diffusivity in the vertical direction prevents overhang of the graphs. We shall explain this point of view in [24]. For equations having very strong diffusivities with inhomogeneous effect we refer to [16] and review articles [32], [21] where variational approach is emphasized.

We now explain physical background of (1.6) and (1.7). The equation (1.6) is considered as a crude model of epitaxial growth of crystals or continuous model of growth of crystal surface. Imagine that a crystal grows on a flat plate. Let $u$ be the height of crystal from the plate. Assume that the crystal grows only horizontally and that the normal velocity $v$ of the each $y$-level set of $u$ depends only on its level and normal i.e.

$$
v=a(y, \mathbf{n}) \quad \text { on }\{u=y\}
$$

This equation is formally equivalent to (1.6). In a crystal growth problem it often happens that the growth speed of higher level sets of $u$ may be faster than lower level set of $u$ in some normal direction because of different structure of crystal. It often happens that $a(y, \mathbf{n})$ is periodic in $y$. Keeping that $u$ as the graph of a function corresponds to the requirement that overhang of crystal is not allowed. Proper solutions give an interpretation of (1.14) under such a requirement. The reader is referred to a very nice article by I. Sunagawa and P. Bennema [39] for such phenomena. The set of jump discontinuities of $u$ is considered as bunching.

The equation (1.7) is of the form

$$
V=b(y) \quad \text { on } \Gamma_{t},
$$


where $\Gamma_{t}$ is the graph of $y=u(x, t)$. This is a typical crystal growth equation with no curvature effect. The requirement is that $\Gamma_{t}$ does not overhang in the direction normal to $y$-axis so we consider (1.13) instead of (1.15).

In this paper we restrict ourselves to first order equations. However, our theory has a potential applicability to second order parabolic equations whose solution may develop jump discontinuities. There are several interesting examples of such equations; see [1], [22], [31], [28], [34] and papers cited there. In particular, it is shown in [22] that a solution $u$ of the equation corresponding to $V=y+k$ may not stay as the graph of a continuous function, where $k$ is the curvature of the graph $y=u(x, t), x \in \mathbf{R}$. A suitable extension of the notion of proper viscosity solutions might give a way to extend such a solution after it develops jump discontinuities (cf. [24]). We postpone to study these problems in one of our forthcoming papers.

This paper is organized as follows. In $\S 2$ we give a definition of proper viscosity and in $\S 3$ we study basic properties of subgraphs. In $\S 4$ we establish the weak comparison principle for (1.1) including (1.6) and (1.7). In $\S 5$ we prove several proper comparison principles. To handle homogeneous equations we also establish a variant of a weak comparison principle. In $\S 6$ we establish stability properties of $u^{\varepsilon}$ and prove a general form of Theorems 1.1-1.3. In $\S 7$ we discuss the conservation law and study the limits $\lim \sup ^{*} u^{\varepsilon}$ and $\liminf _{*} u^{\varepsilon}$. We prove that the limit $u$ in Corollary 1.5 is actually an entropy solution.

\section{Proper viscosity solutions}

We first recall a usual definition [10], [3], [4] of a viscosity subsolution due to H. Ishii for semicontinuous functions to a Hamilton-Jacobi equation

$$
\partial_{t} u+H(x, t, u, \nabla u)=0 \quad \text { in } \quad Q=\Omega \times(0, T),
$$

where $\Omega$ is a domain in $\mathbf{R}^{n} ; \partial_{t} u$ denotes the time derivative $\partial u / \partial t$ and $\nabla u$ denotes the spatial gradient, i.e., $\nabla u=\left(\partial_{x_{1}} u, \ldots, \partial_{x_{n}} u\right)$ with $\partial_{x_{i}} u=\partial u / \partial x_{i}$. We shall always assume that $H$ is continuous in its variables. For a function $u: Q \rightarrow \mathbf{R} \cup\{ \pm \infty\}$ let $u^{*}$ denote the upper semicontinuous envelope, i.e.

$$
u^{*}(x, t)=\limsup _{\varepsilon \downarrow 0}\{u(y, s) ;|y-s|<\varepsilon,|t-s|<\varepsilon,(y, s) \in Q\}
$$

for $(s, t) \in \bar{Q}$. The function $u$ is called a (viscosity) subsolution of $(2.1)$ if $u^{*}<\infty$ on $\bar{Q}$ and

$$
\partial_{t} \varphi(\hat{x}, \hat{t})+H\left(\hat{x}, \hat{t}, u^{*}(\hat{x}, \hat{t}), \nabla \varphi(\hat{x}, \hat{t})\right) \leq 0
$$


holds for all $((\hat{x}, \hat{t}), \varphi) \in Q \times C^{1}(Q)$ satisfying

$$
\max _{Q}\left(u^{*}-\varphi\right)=\left(u^{*}-\varphi\right)(\hat{x}, \hat{t})
$$

Similarly, $v: Q \rightarrow \mathbf{R} \cup\{ \pm \infty\}$ is called a (viscosity) supersolution of (2.1) if $v_{*}>-\infty$ on $\bar{Q}$ and

$$
\partial_{t} \varphi(\hat{x}, \hat{t})+H\left(\hat{x}, \hat{t}, v_{*}(\hat{x}, \hat{t}), \nabla \varphi(\hat{x}, \hat{t})\right) \geq 0
$$

holds for all $((\hat{x}, \hat{t}), \varphi) \in Q \times C^{1}(Q)$ satisfying

$$
\min _{Q}\left(v_{*}-\varphi\right)=\left(v_{*}-\varphi\right)(\hat{x}, \hat{t})
$$

where $v_{*}$ denotes the lower semicontinuous envelope, i.e., $v_{*}=-(-v)^{*}$. If $u$ is both a sub- and supersolution, we say that $u$ is a (viscosity) solution. We shall often suppress the word 'viscosity'. As well-known one may replace max or min as local maximum or minimum, or strict maximum or strict minimum. Also one may replace the class $C^{1}(Q)$ by $C^{\infty}(Q)$.

Such a notion of solution easily extends to second order parabolic problems. For example $u$ is called a subsolution of

$$
\partial_{t} u+H(x, t, u, \nabla u)=\varepsilon \Delta u
$$

if (2.2) is replaced by

$$
\partial_{t} \varphi(\hat{x}, \hat{\imath})+H\left(\hat{x}, \hat{t}, u^{*}(\hat{x}, \hat{t}), \nabla \varphi(\hat{x}, \hat{t})\right)-\varepsilon \Delta \varphi(\hat{x}, \hat{t}) \leq 0
$$

in the definition of subsolution of $(2.1)$ and $C^{1}(Q)$ is replaced by $C^{\infty}(Q)$ or $C^{2}(Q)$; here $\varepsilon$ is a positive constant. By parabolicity it is clear that a classical subsolution $\left(C^{2,1}\right.$-subsolution) is a viscosity subsolution.

When $r \mapsto H(\cdot, \cdot, r, \cdot)$ is nondecreasing, it is well-known that a comparison principle holds under some regularity assumption for $H$. If the periodic boundary condition is imposed and $H$ is independent of $x$, the comparison principle is easy to state as follows. (The nondecreasing condition for $H$ can be slightly weakened [10], [8] ...; however, some monotonicity assumption is necessary to derive the following comparison principle.)

CP. Let $u$ and $v$ be sub- and supersolutions of (2.1) in $Q$ with $\Omega=\mathbf{R}^{n}$ and periodic in space variables with the same period $\left(\omega_{1}, \cdots, \omega_{n}\right)$. If $u^{*} \leq v_{*}$ at $t=0$, then $u^{*} \leq v_{*}$. 
Combining Perron's method [30] with CP, we see that there is a unique global continuous viscosity solution for any periodic continuous initial data. In fact, spatially periodic solution $u$ starting with continuous periodic initial data $u_{0}$ must be continuous in $\mathbf{R}^{n} \times[0, T)$. This follows from $\mathbf{C P}$ since $\mathbf{C P}$ implies $u^{*} \leq u_{*}$. The reader is referred to books [3], [4] for details.

However, if $r \mapsto H(\cdot, \cdot, r, \cdot)$ is not nondecreasing, we do not expect that the solution stays continuous for time interval $(0, T)$ even if initially it is continuous. A familiar example is the Burgers equation $\partial_{t} u+u \partial_{x} u=0, x \in \mathbf{R}$. Even if initial data is smooth, the solution may become discontinuous in finite time so it is not expected that a continuous viscosity solution exists globally in time. In particular we do not expect $\mathbf{C P}$ in the present form. We shall restrict the class of viscosity solutions so that it satisfies a kind of comparison principle which is different from CP. (If CP holds, then it again yields a continuity of solution, so our expected comparison principle must be different from CP.)

We prepare several notions to introduce a restricted viscosity subsolution which we call a proper viscosity subsolution.

Convexification. Let $f$ be a function defined on $\mathbf{R}$. Let $I$ be a bounded closed interval. Let $f_{I}$ denote the convex hull (convexification) of $f$ in $I$ i.e., $f_{I}$ is the greatest convex function on $I$ less than or equal to $f$ in $I$. By definition $f_{I}=f$ on $I$ if $I$ is a singleton.

Lemma 2.1. (i) If $f$ is continuous in $I$, then $f_{I}=f$ on $\partial I$ and $f_{I}$ is continuous in $I$.

(ii) If $f$ is $C^{1}$ in $I$, then $f_{I}$ is $C^{1}$ in $I$.

(iii) For $f \in C^{1}[a, d](-\infty<a<d<\infty)$

$$
f_{I}^{\prime}(x) \geq f_{J}^{\prime}(x) \text { for } x \in I \cap J
$$

with $I=[a, b], J=[c, d], a \leq c \leq b \leq d$, where' denotes the derivative. (At the boundary, the derivative is interpreted as right or left derivative.)

(iv) For $f \in C^{1}(\mathbf{R})$ the function $F(a, b, x)=f_{[a, b]}^{\prime}(x)$ is continuous in $\{(p, q, x) \in$ $\left.\mathbf{R}^{3} ; p \leq q, p \leq x \leq q\right\}$.

Proof. The proof is elementary. However, we give it for completeness.

(i) If $I$ is a singleton, the result is clear. We may assume that $I=[a, b]$ with $-\infty<a<b<\infty$. Since $f$ is continuous, there is a concave modulus $\omega$ such that 
$f(x)-f(a) \geq-\omega(|x-a|)$ for $x \in I$. In particular $f_{I}(a)=f(a)$ and $f_{I}$ is continuous at $a$. The proof at $x=b$ is the same. Since $f_{I}$ is continuous in the interior of $I$, these observations yield the continuity of $f_{I}$ on $I$.

(ii) If $f$ is right differentiable at $x=a$, then for any $\delta>0, f(x) \geq f(a)+$ $\left(d^{+} f(a) / d x-\delta\right)(x-a)$ for $x$ close to $a$. Thus as in (i)

$$
f(x) \geq f_{I}(x) \geq f(a)+\left(d^{+} f(a) / d x-M\right)(x-a), \quad x \in I
$$

for some $M>\delta$ so that $d^{+} f_{I}(a) / d x$ is finite. Since $f_{I}$ is convex and continuous on $I$, $d^{+} f_{I} / d x$ is continuous at $x=a$. Similarly, the left derivative $d^{-} f_{I} / d x$ is continuous at $x=b$.

It remains to prove that $d^{+} f_{I} / d x=d^{-} f_{I} / d x$ on $(a, b)$. If not, there is a point $x_{0}$ such that $p=d^{+} f_{I}\left(x_{0}\right) / d x>d^{-} f_{I}\left(x_{0}\right) / d x=q$. There is a sequence $x_{j} \rightarrow x_{0}$ such that $f\left(x_{j}\right) \geq f_{I}\left(x_{j}\right), f\left(x_{j}\right) \rightarrow f_{I}\left(x_{0}\right)$ since otherwise it contradicts maximality of $f_{I}$ if $p>q$. By continuity $f_{I}\left(x_{0}\right)=f\left(x_{0}\right)$. Since $f(x) \geq f\left(x_{0}\right)+p\left(x-x_{0}\right)$ of $x>x_{0}$ and $f(x) \geq f\left(x_{0}\right)+q\left(x-x_{0}\right)$ for $x \leq x_{0}$ with $p>q$. This contradicts the differentiability of $f$. (The $C^{1}$ regularity assumption on $f$ can be replaced by differentiability of $f$ on $I$.)

(iii) We first prove the case when $a=c$. Let $\tilde{f}$ be

$$
\tilde{f}(x)= \begin{cases}f_{I}(x), & x \in I, \\ f(x), & x \in \mathbf{R} \backslash I .\end{cases}
$$

By definition of convex hull we see that $f_{I} \geq f_{J}$ on $I$ so that $\tilde{f}_{J}=f_{J}$ on $J$. Unless $\tilde{f}=f_{J}$ on $I$, there is $x_{0} \in[a, b)$ such that

$$
\begin{aligned}
& \tilde{f}(x)=f_{J}(x) \quad \text { for } \quad x \in\left[a, x_{0}\right] \\
& \tilde{f}(x)>f_{J}(x) \text { for } \quad x \in\left(x_{0}, b\right] .
\end{aligned}
$$

and that $f_{J}^{\prime}(x)$ is constant for $x \in\left(x_{0}, b\right]$ by definition of convex hull. Thus

$$
f_{J}^{\prime}(x)=f_{J}^{\prime}\left(x_{0}\right) \leq \tilde{f}^{\prime}\left(x_{0}\right) \text { for } x \in\left[x_{0}, b\right] .
$$

Since $\tilde{f}^{\prime}$ is nondecreasing, this implies $f_{J}^{\prime}(x) \leq \tilde{f}^{\prime}(x)$ for $x \in[a, b)$. We have thus proved that $f_{J}^{\prime}(x) \leq f_{I}^{\prime}(x)$ for $x \in I$ by continuity of $f_{I}^{\prime}$ and $f_{J}^{\prime}$.

The proof for $b=d$ is symmetric. Using these two cases we obtain

$$
f_{I}^{\prime}(x) \geq f_{I \cap J}^{\prime}(x) \geq f_{J}^{\prime}(x), \quad x \in I \cap J .
$$

(iv) We first observe that $f_{I_{j}} \uparrow f_{I}(j \rightarrow \infty)$ if $b_{j} \downarrow b$ and $I_{j}=\left[a, b_{j}\right]$ and $I=[a, b]$. Indeed $f_{I_{j}} \leq f_{I_{j+1}}$ on $I_{j+1}$ and $f_{I_{j}} \leq f_{I}$ by definition. If $\lim _{j \rightarrow \infty} f_{I_{j}}=h$ is strictly 
smaller than $f_{I}$ at some point $x_{0} \in[a, b]$, then $h(x)<f_{I}(x)$ for all $x \in\left[x_{0}, b\right]$ since otherwise it would contradict maximality of $f_{I_{j}}$ for large $j$. This is absurd since $h(b)$ must equal $f_{I}(b)=f(b)$. Thus $f_{I_{j}} \uparrow f_{I}$. We also observe that $f_{I_{j}} \downarrow f_{I}$ if $a_{j} \downarrow a$ and $I_{j}=\left[a_{j}, b\right]$ and $I=[a, b]$. Indeed as above $f_{I_{j}} \downarrow$ and $f_{I} \leq f_{I_{j}}$. If the convex function $\lim f_{I_{j}}=h$ is strictly larger than $f_{I}$ this would contradict maximality of $f_{I}$ since $h(a)=f(a)=f_{I}(a)$.

By these convergence results we see that $f_{I_{j}} \rightarrow f_{I}$ for $I_{j}=\left[a_{j}, b_{j}\right]$ when $a_{j} \downarrow a$, $b_{j} \downarrow b$ since $f_{\left[a, b_{j}\right]} \leq f_{I_{j}} \leq f_{\left[a_{j}, b\right]}$. The convergence is uniform in $I$ since $f$ is $C^{1}$ so that $\left|f_{I_{1}}^{\prime}\right|$ is bounded. Since $f_{I}^{\prime}$ is continuous and $f_{I_{j}}$ 's is convex, $f_{I_{j}}^{\prime}$ converges to $f_{I}^{\prime}$ uniformly on $I$ (see e.g. [17, Appendix]).

A symmetric argument yields the uniform convergence

$$
f_{I_{j}}^{\prime} \rightarrow f_{I}^{\prime} \text { on } I
$$

for $a_{j} \uparrow a, b_{j} \uparrow b$ where $I_{j}=\left[a_{j}, b_{j}\right]$

If $a_{j} \downarrow a$ and $b_{j} \uparrow b$, by (iii) we see

$$
f_{\left[a, b_{j}\right]}^{\prime} \geq f_{I_{j}}^{\prime} \geq f_{\left[a_{j}, b\right]}^{\prime}
$$

As we see above two paragraphs, $f_{\left[a, b_{j}\right]}^{\prime} \rightarrow f_{I}^{\prime}$ and $f_{\left[a_{j}, b\right]}^{\prime} \rightarrow f_{I}^{\prime}$ uniformly on $I$, so that $f_{I_{j}}^{\prime}$ converges to $f_{I}^{\prime}$ uniformly on $I$. The remaining case $a_{j} \uparrow a$ and $b_{j} \downarrow b$ can be treated similarly so we conclude that

$$
f_{I_{j}}^{\prime} \rightarrow f_{I}^{\prime}
$$

uniformly on $I$ as $j \rightarrow \infty$, whenever $I_{j}$ converges to $I$. This yields the continuity of $F(a, b, x)$.

Test surface for shock. Let $u$ be upper semicontinuous in $Q$. Let $\left\{S_{t}\right\}_{t \in \Lambda}$ be a smooth family of a smooth hypersurface in $\mathbf{R}^{n}$ such that it contains $x_{0}$ as an interior point of $S_{t_{0}}$, where $\Lambda$ is an open interval in $(0, T)$ containing $t_{0}$. Let $\mathbf{n}$ denote its unit normal vector fields of $S_{t}$ which gives the orientation. Let $B_{r}\left(x_{0}\right)$ denote the closed ball of radius $r$ centered at $x_{0}$ in $\mathbf{R}^{n}$. Let int $B_{r}\left(x_{0}\right)$ denote its interior. Let $\mu$ be a number that satisfies $\mu<u\left(x_{0}, t_{0}\right)$. Suppose that there are numbers $r>0$ and $\delta>0$ such that

$$
u(x, t) \leq \mu \text { on } D_{t} \times\{t\}
$$

for all $t \in\left(t_{0}-\delta, t_{0}+\delta\right) \subset(0, T)$, where $D_{t}$ is an open set in $B_{r}\left(x_{0}\right)$ such that $\partial D_{t}=S_{t}$ in int $B_{r}\left(x_{0}\right)$ and its inward normal agrees with $\mathbf{n}$. If these conditions are 
fulfilled, we say that $\left\{S_{t}\right\}$ is a upper test surface of $u$ at jump discontinuity $\left(x_{0}, t_{0}\right)$ with level $\mu$. If $u(\cdot, t)$ jumps accross a hypersurface $\Sigma_{t}$, such surface $\Sigma_{t}$ is often called shock surfaces. In this case one may take $\Sigma_{t}$ as a test surface if $\Sigma_{t}$ is enough regular.

For lower semicontinuous function $v$ in $Q$ we similarly define a lower test surface. Let $\mu$ be a number satisfying $\mu>v\left(x_{0}, t_{0}\right)$. If there are numbers $r>0$ and $\delta>0$ such that

$$
v(x, t) \geq \mu \text { on } D_{t} \times\{t\}
$$

for all $t \in\left(t_{0}-\delta, t_{0}+\delta\right)$, then $\left\{S_{t}\right\}$ is called a lower test surface of $v$ at jump discontinuity $\left(x_{0}, t_{0}\right)$ with level $\mu$. Here the definition of $D_{t}$ is the same as for upper test surfaces. The inward unit normal vector field of $S_{t}=\partial D_{t}$ is still denoted by $\mathbf{n}$.

For later convenience let $V=V\left(x_{0}, t_{0}\right)$ be the normal velocity of $S_{t_{0}}$ at $x_{0}$ is the direction of $\mathbf{n}\left(x_{0}, t_{0}\right)$.

Relaxed Hamiltonian. We consider the recession function of $H: Q \times \mathbf{R} \times \mathbf{R}^{n} \rightarrow$ $\mathbf{R}$, i.e.,

$$
H_{\infty}(x, t, r, p)=\lim _{\lambda \downarrow 0} \lambda H(x, t, r, p / \lambda) .
$$

We always assume that $H_{\infty}$ exists and continuous in its variables. Let $f(r)$ be a primitive of $H_{\infty}(x, t, r, p)$ as a function of $r$. For a closed inverval $I$ let $f_{I}$ denote the convexification of $f$ in $I$. Since $f_{I}$ is $C^{1}$ in $I$ by Lemma 2.1 (ii) so that $f_{I}^{\prime}$ is continuous on $I$, we set

$$
I^{I}(x, t, r, p)=f_{I}^{\prime}(r), \quad r \in I
$$

and call a relaxed Hamiltonian in $I$. Evidently, $H^{I}$ is independent of the choice of the primitive $f$ so it is well defined. Since $H_{\infty}$ is positively homogeneous of degree one, so is $H^{I}$, i.e., $H^{I}(x, t, r, \sigma p)=\sigma H^{I}(x, t, r, p)$ for all $\sigma>0,(x, t) \in Q, r \in \mathbf{R}$, $p \in \mathbf{R}^{n}$. If $r \mapsto H(x, t, r, p)$ is nondecreasing so that $f(r)$ is convex, the relaxed $H^{I}$ agrees with $H_{\infty}$.

Definition 2.2(Proper solutions). Let $u$ be a subsolution of (2.1) in $Q$. We say that $u$ is a proper subsolution if for any $\left(x_{0}, t_{0}\right) \in Q$ the inequality

$$
V\left(x_{0}, t_{0}\right)+H^{I}\left(x_{0}, t_{0}, u^{*}\left(x_{0}, t_{0}\right),-\mathbf{n}\left(x_{0}, t_{0}\right)\right) \leq 0
$$

with $I=\left[\mu, u^{*}\left(x_{0}, t_{0}\right)\right]$ holds for all upper test surface $\left\{S_{t}\right\}$ of $u^{*}$ at $\left(x_{0}, t_{0}\right)$ with level $\mu\left(<u^{*}\left(x_{0}, t_{0}\right)\right)$. Here $V=V\left(x_{0}, t_{0}\right)$ denotes the normal velocity of $\left\{S_{t}\right\}$ at $\left(x_{0}, t_{0}\right)$ in the direction of $\mathbf{n}\left(x_{0}, t_{0}\right)$ and $H^{I}$ denotes the relaxed Hamiltonian. (By definition a continuous subsolution in $Q$ is always a proper subsolution.) 
For a supersolution $v$ of (2.1) in $Q$ we say that $v$ is a proper supersolution if for any $\left(x_{0}, t_{0}\right) \in Q$ the inequality

$$
-V\left(x_{0}, t_{0}\right)+H^{I}\left(x_{0}, t_{0}, v_{*}\left(x_{0}, t_{0}\right), \mathbf{n}\left(x_{0}, t_{0}\right)\right) \geq 0
$$

with $I=\left[v_{*}\left(x_{0}, t_{0}\right), \mu\right]$ holds for all lower test surface $\left\{S_{t}\right\}$ of $v_{*}$ at $\left(x_{0}, t_{0}\right)$ with level $\mu$. If $u$ is a proper sub- and supersolution, we say that $u$ is a proper solution.

Remark 2.3. (i) If (2.5) is fulfilled with $I=\left[\mu, u^{*}\left(x_{0}, t_{0}\right)\right]$, then (2.5) holds for all $I^{\prime}=[\mu, \sigma]$ provided that $\sigma \geq u^{*}\left(x_{0}, t_{0}\right)$ by Lemma 2.1 (iii).

(ii) If $\left\{S_{t}\right\}$ is an upper test surface of $u^{*}$ at $\left(x_{0}, t_{0}\right)$ with level $\mu$, it is also an upper test surface with level $\mu^{\prime}$ for any $\mu^{\prime} \in\left[\mu, u^{*}\left(x_{0}, t_{0}\right)\right]$. Thus for a proper subsolution the inequality

$$
V\left(x_{0}, t_{0}\right)+H^{J}\left(x_{0}, t_{0}, u^{*}\left(x_{0}, t_{0}\right),-\mathbf{n}\left(x_{0}, t_{0}\right)\right) \leq 0
$$

with $J=\left[\mu^{\prime}, u^{*}\left(x_{0}, t_{0}\right)\right]$ is valid. By Lemma 2.1 (iv) letting $\mu^{\prime} \uparrow u^{*}\left(x_{0}, t_{0}\right)$ yields

$$
V\left(x_{0}, t_{0}\right)+H_{\infty}\left(x_{0}, t_{0}, u^{*}\left(x_{0}, t_{0}\right),-\mathbf{n}\left(x_{0}, t_{0}\right)\right) \leq 0 .
$$

'This inequality holds for any upper test surfaces $\left\{S_{t}\right\}$ at $\left(x_{0}, t_{0}\right)$.

(iii) Suppose that $r \mapsto H(x, t, r, p)$ is nondecreasing so that $H^{I}=H_{\infty}$ for any $I$. If $(2.7)$ holds for any upper test surface $\left\{S_{t}\right\}$ at $\left(x_{0}, t_{0}\right)$, then (2.5) holds for any $\mu$ by monotonicity of $H$ in $r$. Thus $u$ is a proper subsolution if (2.7) holds and $u$ is a subsolution. In fact if $r \mapsto H(x, t, r, p)$ is nondecreasing, every subsolution is a proper subsolution as stated below in Proposition 2.4.

(iv) As for usual subsolution it is possible to define a solution $u$ in $Q^{\prime}=\Omega \times(0, T]$. For $u: Q^{\prime} \rightarrow \mathbf{R} \cup\{ \pm \infty\}$ we say that $u$ is a proper subsolution of $(2.1)$ in $Q^{\prime}$ if it is a subsolution (2.1) in $Q^{\prime}$ (i.e. (2.2) holds for $((x, t), \varphi) \in Q^{\prime} \times C^{1}\left(Q^{\prime}\right)$ satisfying (2.3) with $Q$ replaced by $\left.Q^{\prime}\right)$ and $(2.5)$ holds for upper test surface $\left\{S_{t}\right\}$ at $\left(x_{0}, t_{0}\right) \in Q^{\prime}$ with level $\mu$. If $t_{0}=T$, the family $\left\{S_{t}\right\}$ should be interpreted smooth in $(T-\delta, T]$.

Proposition 2.4 (Consistency). Assume that $r \mapsto H(x, t, r, p)$ is nondecreasing in $\mathbf{R}$ for all $(x, t) \in Q, p \in \mathbf{R}^{n}$. Assume that $\lambda H(x, t, r, p / \lambda)$ converges to $H_{\infty}$ locally uniformly as $\lambda \rightarrow 0$ in the sense that

$$
\sup _{(x, t, r, p)}\left|\lambda H(x, t, r, p / \lambda)-H_{\infty}(x, t, r, p)\right| \rightarrow 0
$$

as $\lambda \rightarrow 0$, where $(x, t, r, p)$ moves in every compact set in $Q \times \mathbf{R} \times \mathbf{R}^{n}$. If $u$ and $v$ are sub- and supersolutions of (2.1) in $Q$ respectively, then $u$ and $v$ are proper suband supersolution of (2.1). 
Proof. The proof is symmetric for sub- and supersolutions so we only present the proof for a subsolution. By Remark 2.3 (iii) it suffices to prove (2.7). Let $\left\{S_{t}\right\}$ be an upper test surface at $\left(x_{0}, t_{0}\right)$ of $u^{*}$ with level $\mu$. Let $D_{t}$ be as in the definition of $S_{t}$. We set

$$
D=\bigcup_{t \in \Lambda} D_{t} \times\{t\} \subset B_{r}\left(x_{0}\right) \times \Lambda, \quad \Lambda=\left(t_{0}-\delta, t_{0}+\delta\right)
$$

We take another upper test function $\left\{S_{t}^{\prime}\right\}$ with level $\mu$ at $\left(x_{0}, t_{0}\right)$ such that

$$
\left(x_{0}, t_{0}\right) \in S^{\prime} \text { and } S^{\prime} \backslash\left\{\left(x_{0}, t_{0}\right)\right\} \subset D \quad \text { with } S^{\prime}=\bigcup_{t \in \Lambda} S_{t}^{\prime} \times\{t\}
$$

(By constructions $S_{t}^{\prime}$ touchs $S_{t}$ only at time $t_{0}$ at point $x_{0}$.) Let $D_{t}^{\prime}$ denote the open set corresponding to $S_{t}^{\prime}$ such that $\partial D_{t}^{\prime}=S_{t}^{\prime}$ in $\operatorname{int} B_{r}\left(x_{0}\right)$. To construct an appropriate test function for $u^{*}$ we use a signed distance function of $D^{\prime}=\cup_{t \in \Lambda} D_{t}^{\prime} \times\{t\} \subset$ $B_{r}\left(x_{0}\right) \times \Lambda$ defined by

$$
d(x, t)= \begin{cases}\operatorname{dist}\left((x, t), \partial D^{\prime}\right), & x \in D^{\prime} \\ -\operatorname{dist}\left((x, t), \partial D^{\prime}\right), & x \notin D^{\prime}\end{cases}
$$

Since $\partial D$ is smooth, so is $d$ in $B_{r}\left(x_{0}\right) \times \Lambda$ for sufficiently small $\delta$ and $r$. We fix $\mu^{\prime} \in\left(\mu, u^{*}\left(x_{0}, t_{0}\right)\right)$ and define

$$
\varphi_{L}(x, t)=\max \left(-L d(x, t)+u^{*}\left(x_{0}, t_{0}\right), \mu^{\prime}\right)
$$

for $L>0$. The function $\varphi_{L}(x, t)$ is smooth outside $D^{\prime}$ in a small neighborhood of $\left(x_{0}, t_{0}\right)$. Since $u^{*}$ is upper semicontinuous, there is a maximizer $\left(x_{L}, t_{L}\right)$ of $u^{*}-\varphi_{L}$ in $B_{r}\left(x_{0}\right) \times \bar{\Lambda}$. Sending $L \rightarrow \infty$ we see that $0 \leq \max \left(u^{*}-\varphi_{L}\right) \rightarrow 0$ and $\operatorname{dist}\left(\left(x_{L}, t_{L}\right), \partial D^{\prime}\right) \rightarrow 0$. Since $\left(x_{L}, t_{L}\right) \notin D$ this implies $\left(x_{L}, t_{L}\right) \rightarrow\left(x_{0}, t_{0}\right)$. Moreover, $u^{*}\left(x_{L}, t_{L}\right) \rightarrow u^{*}\left(x_{0}, t_{0}\right)$ since $u^{*}$ is upper semicontinuous and $u^{*}\left(x_{L}, t_{L}\right) \geq$ $u^{*}\left(x_{0}, t_{0}\right)$. Thus for sufficiently large $L$ the function $u^{*}-\varphi_{L}$ takes its local maximum at $\left(x_{L}, t_{L}\right) \in$ int $B_{r}\left(x_{0}\right) \times \Lambda$ and at $\left(x_{L}, t_{L}\right)$ the function $\varphi_{L}$ is smooth.

If $u$ is a subsolution, then

$$
\partial_{t} \varphi\left(x_{L}, t_{L}\right)+H\left(\left(x_{L}, t_{L}, u^{*}\left(x_{L}, t_{L}\right), \nabla \varphi_{L}\left(x_{L}, t_{L}\right)\right) \leq 0 .\right.
$$

Dividing by $\left|\nabla \varphi\left(x_{L}, t_{L}\right)\right|=L$ and sending $L \rightarrow \infty$ yields

$$
V+H_{\infty}\left(x_{0}, t_{0}, u^{*}\left(x_{0}, t_{0}\right),-\mathbf{n}\left(x_{0}, t_{0}\right)\right) \leq 0
$$


since the convergence $\lambda H(x, t, r, p / \lambda) \rightarrow H_{\infty}(x, t, r, p)$ is locally uniform in $(x, t, r, p)$ as $\lambda \rightarrow 0$ and

$$
\begin{aligned}
& \frac{\nabla \varphi_{L}\left(x_{L}, t_{L}\right)}{\left|\nabla \varphi_{L}\left(x_{L}, t_{L}\right)\right|} \rightarrow-\mathbf{n}\left(x_{0}, t_{0}\right) . \quad\left(x_{L}, t_{L}\right) \rightarrow\left(x_{0}, t_{0}\right) \\
& u^{*}\left(x_{L}, t_{L}\right) \rightarrow u^{*}\left(x_{0}, t_{0}\right) . \quad \frac{\partial_{t} \varphi\left(x_{L}, t_{L}\right)}{\left|\nabla \varphi\left(x_{L}, t_{L}\right)\right|} \rightarrow V\left(x_{0}, t_{0}\right) .
\end{aligned}
$$

We have thus proved (2.7).

Examples. We consider the Riemann problem for the scalar conservation law in one space dimension of the form:

$$
\partial_{t} u+\partial_{x}(f(u))=0, \quad x \in \mathbf{R}, \quad t>0
$$

Here $f$ is assumed to be a given $C^{1}$ strictly convex function. The equation (2.9) can be written in the form of (2.1) with

$$
H(x, t, r, p)=H(r, p)=f^{\prime}(r) p .
$$

A typical example is the Burgers equation where $f(r)=r^{2} / 2$. For $a<b$, the function

$$
u_{E}(x, t)=\left\{\begin{array}{ll}
b, & x \leq c t, \\
a, & x>c t,
\end{array} \quad u_{N}(x, t)= \begin{cases}a, & x<c t \\
b, & x \geq c t\end{cases}\right.
$$

solves (2.9) in the distribution sence provided that the speed $c$ of the shock satisfies the Rankine-Hugoniot condition:

$$
c=\frac{f(b)-f(a)}{b-a}
$$

However, it is well-known that $u_{E}$ is an entropy solution while $u_{N}$ is not an entropy solution [12]. In other words $u_{E}$ solves

$$
\partial_{t}(\eta(u))+\partial_{x}(q(u)) \leq 0
$$

in the distribution sense for any entropy pair $(\eta, q)$, i.e. $\eta$ is a convex function satisfying $q^{\prime}=\eta^{\prime} f^{\prime}$. While $u_{N}$ does not satisfy (2.11) for some $(\eta, q)$. (Note that for $C^{1}$ solution $u$ of $(2.9)$ the equality version of $(2.10): \partial_{t}(\eta(u))+\partial_{x}(q(u))=0$ holds by definition of $(\eta, q)$. So $C^{1}$-solution is always an entropy solution.) We shall show that our notion of proper solutions also distinguish an admissible shock profile i.e. a shock profile of an entropy solution from a non-admissible one. 
Proposition 2.5. The function $u_{E}$ is a proper solution while $u_{N}$ is not a proper solution of (2.9). (Both $u_{E}$ and $u_{N}$ are solution of (2.9) in usual viscosity sense.)

Proof. It is easy to see that $u_{E}$ and $u_{N}$ are viscosity solutions. So it suffices to check the speed of a test surface for shocks. Let $\left(x_{0}, t_{0}\right)$ be a point on jumps, i.e., $x_{0}=c t_{0}, t_{0}>0$. The line $S_{t}=\{x=c t\}$ itself is a test surface of $u_{E}$ and $u_{N}$ at $\left(x_{0}, t_{0}\right)$ with level $a$. All other test surface at $\left(x_{0}, t_{0}\right)$ is tangent to $\left\{S_{t}\right\}$ so by Remark 2.3 (ii) it suffices to estimate the normal velocity of $\left\{S_{t}\right\}$. If we consider $u_{E}$, then $\mathbf{n}=1$ so that

$$
H(r,-\mathbf{n})=-f^{\prime}(r)
$$

by (2.10). Since $-f$ is concave,

$$
\frac{d}{d r}(-f)_{I}(r)=-\frac{f(b)-f(a)}{b-a}, \quad r \in I=[a, b]
$$

which yields $H^{I}(r,-1)=-c$ by the definition of $c$. Since $V\left(x_{0}, t_{0}\right)=c$, we now observe that

$$
V\left(x_{0}, t_{0}\right)+H^{I}(b,-1)=0 .
$$

Thus $u_{E}$ is a proper subsolution. A symmetric argument shows that $\left(u_{E}\right)_{*}$ is a proper supersolution.

We now consider $u_{N}$. In this case the normal $\mathbf{n}$ of $\left\{S_{t}\right\}$ should be taken $\mathbf{n}=-1$ so that $H(r,-\mathbf{n})=f^{\prime}(r)$ by $(2.10)$. Since $f$ is convex, $H^{I}(r,-\mathbf{n})=f^{\prime}(r)=H(r,-\mathbf{n})$ with $I=[a, b]$. Since $V\left(x_{0}, t_{0}\right)=-c$, we now observe that

$$
V\left(x_{0}, t_{0}\right)+H^{I}(b,-\mathbf{n})=-c+f^{\prime}(b)>0
$$

by strictly convexity of $f$. Thus $u_{N}$ is not a proper subsolution (A symmetric argument shows that $u_{N}$ is not a proper supersolution.)

It is well-known that the entropy solution $u$ with initial data $\left.u\right|_{t=0}=\left.u_{N}\right|_{t=0}$ is a rarefaction wave solution:

$$
u_{R}(x, t)= \begin{cases}a, & x<f^{\prime}(a) t \\ \left(f^{\prime}\right)^{-1}(x / t), & f^{\prime}(a) t \leq x<f^{\prime}(b) t \\ b, & x \geq f^{\prime}(b) t\end{cases}
$$

where $f^{\prime-1}$ denotes the inverse function of $f^{\prime}$. This function $u$ is a continuous viscosity solution so there are no jumps. So there are no test surface for shocks. Thus $u_{R}$ is automatically a proper solution. In $\S 7$ we shall show that notions of proper and entropy solutions essentially agree each other for initial-value problems. 


\section{Graphs of proper solutions}

We shall discuss several properties of subgraph $\{(x, z, t) ; z \leq u(x, t)\}$ for proper subsolutions to prove weak comparison principle stated in the next section.

We first recall properties which follows from accessibility in [9]. The accessibility property is valid for every subsolution which may not be proper. For a function $u$ we associate a function on $\Omega \times \mathbf{R} \times(0, T)$ of form

$$
i_{u}(x, z, t)= \begin{cases}0, & z \leq u(x, t) \\ -\infty, & z>u(x, t) .\end{cases}
$$

The set $\left\{i_{u}=0\right\}$ is called a subgraph of $u$ and denoted sg $u$. Similarly we define

$$
I_{u}(x, z, t)= \begin{cases}0, & z \geq u(x, t), \\ +\infty, & z<u(x, t) .\end{cases}
$$

The set $\left\{I_{u}=0\right\}$ is called a supergraph of $u$ and denoted Sg $u$. This set is usually called the epigraph of $u$ and $I_{u}$ is called the indicator function of $\mathrm{Sg} u$ in convex analysis. By definition $\operatorname{sg} u$ is closed if and only if $u$ is upper semicontinuous. The closure of $\operatorname{sg} u$ equals the subgraph of $u^{*}$, i.e. $\overline{\operatorname{sg} u}=\operatorname{sg} u^{*}$. By definition $i_{u^{*}}=\left(i_{u}\right)^{*}$ so $i_{u^{*}}$ is always upper semicontinuous.

Proposition 3.1. Let $u$ be a subsolution of (2.1) in $Q=\Omega \times(0, T)$.

(i) $u^{*}$ is right accessible at each $\left(x_{0}, t_{0}\right) \in Q$, i.e., a sequence $\left\{\left(x_{j}, t_{j}\right)\right\}_{j=1}^{\infty} \subset Q$ such that $x_{j} \rightarrow x_{0}, t_{j} \uparrow t_{0}, u^{*}\left(x_{j}, t_{j}\right) \rightarrow u^{*}\left(x_{0}, t_{0}\right)$ as $j \rightarrow \infty$.

(ii) $i_{u^{*}}$ is right accessible as a function in $\hat{Q}=\Omega \times \mathbf{R} \times(0, T)$.

(iii) If $i_{u^{*}}-\Phi$ takes its maximum over $\hat{Q}$ at $\left(x_{0}, z_{0}, t_{0}\right)$ for $\Phi \in C^{1}(\hat{Q})$, then $\partial_{t} \Phi\left(x_{0}, z_{0}, t_{0}\right) \leq 0$ provided that

$$
\partial_{x_{j}} \Phi\left(x_{0}, z_{0}, t_{0}\right)=0, \quad j=1, \cdots, n \quad \text { and } \partial_{z} \Phi\left(x_{0}, z_{0}, t_{0}\right)=0 \text {, }
$$

i.e., the spatial gradient $\hat{\nabla} \Phi=\left(\nabla_{x} \Phi, \partial_{z} \Phi\right)$ of $\Phi$ vanishes at $\left(x_{0}, z_{0}, t_{0}\right)$.

Remark 3.2. (i) Of course, a symmetric statement holds for a supersolution $v$ of (2.1). For example if $I_{v_{*}}-\Phi$ takes its minimum over $\hat{Q}$ at $\left(x_{0}, z_{0}, t_{0}\right)$, then $\partial_{t} \Phi\left(x_{0}, z_{0}, t_{0}\right) \geq 0$ provided that $\hat{\nabla} \Phi\left(x_{0}, z_{0}, t_{0}\right)=0$. It is easy to observe this fact from the proof below.

(ii) The statement of Proposition 3.1 is still valid even if we replace $Q$ by $\Omega \times(0, T]$ and $\hat{Q}$ by $\Omega \times \mathbf{R} \times(0, T]$.

Proof. (i) This is a special case of the accessibility theorem proved in [9]. 
(ii) This easily follows from (i).

(iii) We may assume that $\Phi$ is of form $\Phi(x, z, t)=\psi(x, z)+a(t)$ near $\left(x_{0}, z_{0}, t_{0}\right)$. Since $i_{u^{*}}$ is constant on $\mathrm{sg} u^{*}$ and equals $-\infty$ outside $\operatorname{sg} u^{*}$, we may also assume that $\psi$ is constant near $\left(x_{0}, z_{0}\right)$. Suppose that $\partial_{t} \Phi\left(x_{0}, z_{0}, t_{0}\right)>0$ so that $a^{\prime}\left(t_{0}\right)>0$. Since $i_{u^{*}}-\Phi$ takes maximum at $\left(x_{0}, z_{0}, t_{0}\right)$ and $a^{\prime}\left(t_{0}\right)>0$, we see that $i_{u^{*}}(x, z, t)<$ 0 for $(x, z, t)$ near $\left(x_{0}, z_{0}, t_{0}\right)$ with $t<t_{0}$. Thus $i_{u^{*}}(x, z, t)=-\infty$ there and $i_{u^{*}}\left(x_{0}, z_{0}, t_{0}\right)=0$. This is absurd since $i_{u^{*}}$ must be right accessible at $\left(x_{0}, z_{0}, t_{0}\right)$ by (ii). We have thus proved that $\partial_{t} \Phi\left(x_{0}, z_{0}, t_{0}\right) \leq 0$.

Lemma 3.3. Let $u$ be a proper subsolution of (2.1) in $Q=\Omega \times(0, T)$. Let $\Phi$ be $C^{\infty}$ in $\hat{Q}=\Omega \times \mathbf{R} \times(0, T)$. Assume that $(\hat{x}, \hat{z}, \hat{t}) \in \hat{Q}$ is a maximizer of $i_{u^{*}}-\Phi$, i.e.,

$$
\max _{\hat{Q}}\left(i_{u^{*}}-\Phi\right)=\left(i_{u^{*}}-\Phi\right)(\hat{x}, \hat{z}, \hat{t})
$$

Assume that $\hat{\nabla} \Phi(\hat{x}, \hat{z}, \hat{t}) \neq 0$. Then $\hat{z} \leq u^{*}(\hat{x}, \hat{t})$.

(i) If $\partial_{z} \Phi(\hat{x}, \hat{z}, \hat{t}) \neq 0$, then $\hat{z}=u^{*}(\hat{x}, \hat{t})$ and $\partial_{z} \Phi(\hat{x}, \hat{z}, \hat{t})<0$. Moreover,

$$
\tau+H\left(\hat{x}, \hat{t}, u^{*}(\hat{x}, \hat{t}), p\right) \leq 0
$$

with $\tau=-\left(\partial_{t} \Phi / \partial_{z} \Phi\right)(\hat{x}, \hat{z}, \hat{t}) \in \mathbf{R}, p=-\left(\nabla_{x} \Phi / \partial_{z} \Phi\right)(\hat{x}, \hat{z}, \hat{t}) \in \mathbf{R}^{n}$.

(ii) If $\partial_{z} \Phi(\hat{x}, \hat{z}, \hat{t})=0$ and $\hat{z}<u^{*}(\hat{x}, \hat{t})$, then

$$
\partial_{t} \Phi(\hat{x}, \hat{z}, \hat{t})+H^{I}\left(\hat{x}, \hat{t}, u^{*}(\hat{x}, \hat{t}), \nabla_{x} \Phi(\hat{x}, \hat{z}, \hat{t})\right) \leq 0 \text { with } I=\left[\hat{z}, u^{*}(\hat{x}, \hat{t})\right] .
$$

(iii) Assume (2.8). Assume that $\partial_{z} \Phi(\hat{x}, \hat{z}, \hat{t})=0$ and $\hat{z}=u^{*}(\hat{x}, \hat{t})$. Then the inequality (3.5) holds.

All statements still holds even if we replace $Q$ by $\Omega \times(0, T]$ and $\hat{Q}$ by $\Omega \times \mathbf{R} \times$ $(0, T]$.

Remark 3.4. A symmetric statement holds for proper supersolutions.

Proof. Since $\hat{\nabla} \Phi(\hat{x}, \hat{z}, \hat{t}) \neq 0,(\hat{x}, \hat{z}, \hat{t})$ must belong to the boundary of subgraph $\operatorname{sg} u^{*}$. For $\ell=\Phi(\hat{x}, \hat{z}, \hat{t})$ the $\ell$-level set of $\Phi$ touches $\operatorname{sg} u^{*}$ at $(\hat{x}, \hat{z}, \hat{t})$ and the set $\{\Phi<\ell\}$ does not intersect sg $u^{*}$. Clearly, $\hat{z} \leq u^{*}(\hat{x}, \hat{t})$.

(i) The first statement is clear by definition of $\operatorname{sg} u$. Since $\partial_{z} \Phi(\hat{x}, \hat{z}, \hat{t})<0$, the $\ell$-level set of $\Phi$ can be written as the graph of an implicit function $z=z(x, t)$ near $(\hat{x}, \hat{z}, \hat{t})$. By the geometry of $\ell$-level set of $\Phi$ and $\operatorname{sg} u^{*}, u^{*}-z$ takes its local maximum at $(\hat{x}, \hat{t})$. Since $z$ is an implicit function satisfying $\Phi(x, z(x, t), t)=0$, we see $\partial_{t} z(\hat{x}, \hat{t})=\tau$ and $\nabla z(\hat{x}, \hat{t})=p$. Since $u^{*}$ is a subsolution, we obtain (3.4). 
(ii) We may assume that $\Phi(\hat{x}, \hat{z}, \hat{t})=0$ and $\hat{x}=0$. Since $\nabla_{x} \Phi(0, \hat{z}, \hat{t}) \neq 0$, by rotation we may assume that

$$
\nabla_{x} \Phi /\left|\nabla_{x} \Phi\right|=(-1,0, \cdots, 0) \text { at }(0, \hat{z}, \hat{t})
$$

For a suitable choice of $R>0$ and $A \in \mathbf{R}$ a ball $B$ of form

$$
Q(x, z, t)=\left(x_{1}-R\right)^{2}+(z-\hat{z})^{2}+x_{2}^{2}+\cdots+x_{n}^{2}+(t-\hat{t})^{2}+A(t-\hat{t})-R^{2} \leq 0
$$

touches sg $u^{*}$ only at $(0, \hat{z}, \hat{t})$, i.e. $\operatorname{sg} u^{*} \cap B=\{(0, \hat{z}, \hat{t})\}$ and $B \subset\{\Phi \leq 0\}$. Thus $\partial_{t} Q /\left|\nabla_{x} Q\right|=\partial_{t} \Phi /\left|\nabla_{x} \Phi\right|$ at $(0, \hat{z}, \hat{t})$. We now observe that

$$
u(x, t) \leq \hat{z}
$$

for $x \in D_{t}=\{x ; Q(x, \hat{z}, t)<0\}$. We take $S_{t}$ as the boundary of $D_{t}$ and observe that $\left\{S_{t}\right\}$ is an upper test surface with level $\hat{z}$ of $u^{*}$ at $(0, \hat{t})$ and the inward normal $\mathbf{n}$ of $S_{\hat{t}}$ at 0 equals $(1,0, \cdots, 0)=\mathbf{n}$. The normal velocity $V$ of $S_{\hat{t}}$ at 0 equals $\partial_{t} Q /\left|\nabla_{x} Q\right|=\partial_{t} \Phi /\left|\nabla_{x} \Phi\right|(0, \hat{z}, \hat{t})$. Since $u$ is a proper subsolution,

$$
V+H^{I}\left(0, \hat{t}, u^{*}(0, \hat{t}),-\mathbf{n}\right) \leq 0
$$

with $I=\left[\hat{z}, u^{*}(0, \hat{t})\right]$. Noting $V=\partial_{t} \Phi /\left|\nabla_{x} \Phi\right|(0, \hat{z}, \hat{t})$ and $\mathbf{n}=-\nabla_{x} \Phi /\left|\nabla_{x} \Phi\right|(0, \hat{z}, \hat{t})$, we conclude that (3.6) yields (3.5) since $H^{I}(x, t, r, p)$ is positively homogeneous of degree one in $p$.

(iii) We use $Q$ defined in (ii). Let $\tilde{Q}$ be a $C^{1}$ function defined by

$$
\tilde{Q}(x, z, t)= \begin{cases}Q(x, z, t) & \text { if } z \leq \hat{z} \\ Q(x, z, t)-(z-\hat{z})^{2} & \text { if } z>\hat{z}\end{cases}
$$

Since $\operatorname{sg} u^{*}$ is a subgraph, the set $\{\tilde{Q} \leq 0\}$ still touches sg $u^{*}$ only at $(0, \hat{z}, \hat{t})$. For $\varepsilon>0$ we set

$$
\tilde{Q}_{\varepsilon}(x, z, t)=\tilde{Q}(x, z, t)-\varepsilon(z-\hat{z}) .
$$

Let $\left(x_{\varepsilon}, z_{\varepsilon}, t_{\varepsilon}\right)$ be a maximizer of $i_{u^{*}}-\tilde{Q}_{\varepsilon}$. By the convergence of maximum points [4], we see that $\left(x_{\varepsilon}, z_{\varepsilon}, t_{\varepsilon}\right) \rightarrow(0, \hat{z}, \hat{t})$ as $\varepsilon \rightarrow 0$. Since

$$
\partial_{z} \tilde{Q}_{\varepsilon}(x, z, t)=2 \min ((z-\hat{z}), 0)-\varepsilon<0
$$

we apply (i) to get $z_{\varepsilon}=u^{*}\left(x_{\varepsilon}, t_{\varepsilon}\right)$ and

$$
\partial_{t} \tilde{Q}_{\varepsilon}\left(x_{\varepsilon}, z_{\varepsilon}, t_{\varepsilon}\right)+\lambda_{\varepsilon} H\left(x_{\varepsilon}, t_{\varepsilon}, z_{\varepsilon}, \nabla_{x} \tilde{Q}_{\varepsilon}\left(x_{\varepsilon}, z_{\varepsilon}, t_{\varepsilon}\right) / \lambda_{\varepsilon}\right) \leq 0 .
$$


with $\lambda_{\varepsilon}=-\partial_{z} \tilde{Q}_{\varepsilon}\left(x_{\varepsilon}, z_{\varepsilon}, t_{\varepsilon}\right)$ for small $\varepsilon>0$. By (2.8) letting $\varepsilon \rightarrow 0$ yields

$$
\partial_{t} Q(0, \hat{z}, \hat{t})+H_{\infty}\left(0, \hat{t}, \hat{z}, \nabla_{x} Q(0, \hat{z}, \hat{t})\right) \leq 0
$$

since $\lambda_{\varepsilon} \rightarrow 0$ as $\varepsilon \rightarrow 0$. Since $\partial_{t} Q /\left|\nabla_{x} Q\right|=\partial_{t} \Phi /\left|\nabla_{x} \Phi\right|, \nabla_{x} Q /\left|\nabla_{x} Q\right|=\nabla_{x} \Phi /\left|\nabla_{x} \Phi\right|$ at $(0, \hat{z}, \hat{t})$, the inequality $(3.7)$ yields $(3.5)$.

The proof of the statement for $\Omega \times(0, T]$ and $\Omega \times \mathbf{R} \times(0, T]$ is similar so it is safely left to the reader.

\section{Weak comparison principle}

We shall establish a version of the comparison principle which is different from CP in $\S 2$. To avoid technical complexity we consider (2.1) when $H$ is independent of $x$ and $t$, i.e.,

$$
\partial_{t} u+H(u, \nabla u)=0
$$

under a periodic boundary condition. We list our assumptions on $H$.

(H1) $H: \mathbf{R} \times \mathbf{R}^{n} \rightarrow \mathbf{R}$ is continuous

(H2) For each $M>0$ there is a constant $C=C(M)>0$ that satisfies $\mid H(r, p)$ $H\left(r^{\prime}, p\right)|\leq C| r-r^{\prime} \mid(|p|+1)$ for all $r, r^{\prime} \in \mathbf{R}$ satisfying $|r|,\left|r^{\prime}\right| \leq M, p \in \mathbf{R}^{n}$.

(H3) $H_{\infty}$ exists and the convergence $\lambda H(r, p / \lambda)$ to $H_{\infty}$ as $\lambda \rightarrow 0$ is locally uniform in $(r, p) \in \mathbf{R} \times \mathbf{R}^{n}$.

We do not assume that $r \mapsto H(r, p)$ is nondecreasing. The last condition (H3) is nothing but (2.8). The condition (H3) with (H1) yields the continuity of $H_{\infty}$. If $u$ is a proper subsolution of $(4.1)$ in $\mathbf{R}^{n} \times(0, T)$ and $u$ is periodic in all $x_{i}$ 's i.e.,

$$
u(x, t)=u\left(x+\omega_{i} e_{i}, t\right)
$$

with some $\omega_{i}>0$ for all $i=1,2, \cdots, n$, then we say $u$ is a proper subsolution of (4.1) in $\mathbf{T}^{n} \times(0, T)$ with $\mathbf{T}^{n}=\left(\mathbf{R} / \omega_{1} \mathbf{Z}\right) \times \cdots \times\left(\mathbf{R} / \omega_{n} \mathbf{Z}\right)$; here $e_{i}$ is the unit vector in the direction of $x_{i}$-axis. We use similar convention for periodic proper supersolutions.

Theorem 4.1 (Weak comparison principle-WCP). Assume (H1)-(H3). Let $u$ and $v$ be bounded proper sub- and supersolutions of (4.1) in $\mathbf{T}^{n} \times(0, T)$, respectively. If $u^{*}<v_{*}$ at $t=0$, i.e., $u^{*}(x, 0)<v_{*}(x, 0)$ for all $x \in \mathbf{T}^{n}$, then $u^{*}<v_{*}$ in $\mathbf{T}^{n} \times[0, T)$. 
Proof. We may assume that $u$ and $-v$ are upper semicontinuous on $\mathbf{T}^{n} \times[0, T]$ by taking semicontinuous envelope. Let $i_{u}$ and $I_{v}$ be as in (3.1) and (3.2). Suppose that the conclusion were false. Then there would exist $\left(x_{0}, t_{0}\right) \in \mathbf{T}^{n} \times(0, T)$ such that $u\left(x_{0}, t_{0}\right) \geq v\left(x_{0}, t_{0}\right)$. Thus $\left(i_{u}-I_{v}\right)\left(x_{0}, z_{0}, t_{0}\right)$ with $z_{0}=u\left(x_{0}, t_{0}\right)$ is the maximum value 0 of $i_{u}-I_{v}$. Since $i_{u}$ and $-I_{v}$ are upper semicontinuous in $\mathbf{T}^{n} \times \mathbf{R} \times[0, T]$ and $u$ and $v$ are bounded so that

$$
\begin{aligned}
& i_{u}(x, z, t)=-\infty, \quad I_{v}(x, z, t)=0 \quad \text { for } z \geq M \\
& i_{u}(x, z, t)=0, \quad I_{v}(x, z, t)=\infty \quad \text { for } z \leq-M
\end{aligned}
$$

with some $M>\sup |u|, \sup |v|$ independent of $(x, t)$, the function $i_{u}(x, z, t)-$ $I_{v}(x, z, t)-\sigma(T-t)^{-1}$ for $\sigma>0$ takes its maximum $m_{\sigma}(<0)$ at some $\left(x_{0}^{\prime}, z_{0}^{\prime}, t_{0}^{\prime}\right) \in$ $Z_{0}=\mathbf{T}^{n} \times \mathbf{R} \times[0, T)$ over $Z=\mathbf{T}^{n} \times \mathbf{R} \times[0, T]$. We fix $\sigma>0$ and consider

$$
\Psi(X, t, Y, s)=i_{u}(X, t)-I_{v}(Y, s)-\alpha|X-Y|^{2}-\alpha|t-s|^{2}-\sigma(T-t)^{-1}
$$

for $X=(x, z), Y=(y, w) \in \mathbf{T}^{n} \times \mathbf{R}, t, s \in[0, T)$, where $\alpha$ is a positive parameter. Since $\Psi$ is upper semicontinuous in $Z \times Z$ and $u, v$ are bounded, there is a maximizer (depending on $\alpha$ ) $(\hat{X}, \hat{t}, \hat{Y}, \hat{s})=(\hat{X}(\alpha), \hat{t}(\alpha), \hat{Y}(\alpha), \hat{s}(\alpha)) \in Z_{0} \times Z_{0}$ (with $|\hat{z}|,|\hat{w}| \leq$ $M$ ) of $\Psi$ over $Z \times Z$, i.e.,

$$
\max _{Z \times Z} \Psi=\Psi(\hat{X}, \hat{t}, \hat{Y}, \hat{s}) .
$$

Since $\Psi\left(x_{0}^{\prime}, z_{0}^{\prime}, t_{0}^{\prime}, x_{0}^{\prime}, z_{0}^{\prime}, t_{0}^{\prime}\right)=m_{\sigma}, \max \Psi \geq m_{\sigma}$. By this bound we see that $\alpha|\hat{X}-\hat{Y}|^{2}$ and $\alpha|\hat{t}-\hat{s}|^{2}$ is bounded. In particular, $\hat{X}-\hat{Y} \rightarrow 0$ and $|\hat{t}-\hat{s}| \rightarrow 0$ as $\alpha \rightarrow \infty$. Since $u<v$ at $t=0$ so that $i_{u}(X, 0)-I_{v}(X, 0)=-\infty$ and since $\Psi$ is upper semicontinuous, $\hat{t}, \hat{s}$ must be positive for sufficiently large $\alpha$

For later convenience we notice $\alpha\left(|\hat{X}-\hat{Y}|^{2}+|\hat{t}-\hat{s}|^{2}\right) \rightarrow 0$ as $\alpha \rightarrow \infty$. This is standard e.g. [3], [10] but we give a proof for completeness. We set

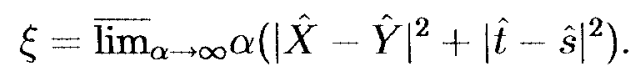

We may assume that $\hat{X} \rightarrow \bar{X}, \hat{Y} \rightarrow \bar{X}, \hat{t} \rightarrow \bar{t}, \hat{s} \rightarrow \bar{t}$ by taking subsequence since $\hat{X}-\hat{Y} \rightarrow 0, \hat{t}-\hat{s} \rightarrow 0$. We may assume that $\alpha\left(|\hat{X}-\hat{Y}|^{2}+|\hat{t}-\hat{s}|^{2}\right) \rightarrow \xi$ by taking a subsequence. Since $i_{u}(X, t)-I_{v}(Y, s)$ is upper semicontinuous,

$$
\begin{aligned}
& i_{u}(\bar{X}, \bar{t})-I_{v}(\bar{X}, \bar{t})-\sigma(T-\bar{t})^{-1} \geq \overline{\lim }_{\alpha \rightarrow \infty}\left(i_{u}(\hat{X}, \hat{t})-I_{v}(\hat{Y}, \hat{s})-\sigma(T-\hat{t})^{-1}\right) \\
& \geq \overline{\lim }_{\alpha \rightarrow \infty} \Psi(\hat{X}, \hat{t}, \hat{Y}, \hat{s})=0-\xi-\sigma(T-\bar{t})^{-1} .
\end{aligned}
$$

Since the leftest hand side is dominated from above by $m_{\sigma}$ and the rightest hand side is dominated from below by $m_{\sigma}$, we see $0-\sigma(T-\bar{t})^{-1}=0-\xi-\sigma(T-\bar{t})^{-1}$, 
which yields $\xi=0$. We have thus proved that

$$
\alpha\left(|\hat{X}-\hat{Y}|^{2}+|\hat{t}-\hat{s}|^{2}\right) \rightarrow 0 \quad \text { as } \alpha \rightarrow \infty .
$$

We note that if $(\hat{X}, \hat{t}, \hat{Y}, \hat{s})$ with $\hat{X}=(\hat{x}, \hat{z}), \hat{Y}=(\hat{y}, \hat{w})$ satisfies $\hat{z}>\hat{w}$, then $(\check{X}, \hat{t}, \hat{Y}, \hat{s})$ is also maximizer of $\Psi$ with $\check{X}=(\hat{x}, \check{z}), \check{z}=\hat{w}-(\hat{z}-\hat{w})$ since $i_{u}(\hat{x}, z, \hat{t})=0$ for $z \leq \hat{z}$. Thus we shall only consider the maximum point $(\hat{X}, \hat{t}, \hat{Y}, \hat{s})$ satisfying $\hat{z} \leq \hat{w}$.

We set

$$
\Xi(X, t, Y, s)=\alpha\left(|X-Y|^{2}+|t-s|^{2}\right)+\sigma(T-t)^{-1}
$$

and divide the situation into two cases.

Case 1. There is a sequence $\alpha=\alpha_{j}$ tending to infinity as $j \rightarrow \infty$ such that

$$
\hat{\nabla}_{X} \Xi(\hat{X}, \hat{t}, \hat{Y}, \hat{s})=0, \quad \hat{\nabla}_{X}=\left(\nabla_{x}, \partial_{z}\right)
$$

with $\hat{X}=\hat{X}\left(\alpha_{j}\right), \ldots$

Case 2. For sufficiently large $\alpha$

$$
\hat{\nabla}_{X} \Xi(\hat{X}, \hat{t}, \hat{Y}, \hat{s}) \neq 0
$$

In the Case 1 we fix $\alpha=\alpha_{j}$. Since $\Psi$ takes its maximum at $(\hat{X}, \hat{t}, \hat{Y}, \hat{s})$, the function

$$
(X, t) \mapsto i_{u}(X, t)-\Xi(X, t, \hat{Y}, \hat{s})-I_{v}(\hat{Y}, \hat{s})
$$

takes its maximum at $(\hat{X}, \hat{t})$. By Proposition 3.1 (iii)

$$
\partial_{t} \Xi(\hat{X}, \hat{t}, \hat{Y}, \hat{s}) \leq 0 .
$$

Similarly, the function

$$
(Y, s) \mapsto I_{v}(Y, s)+\Xi(\hat{X}, \hat{t}, Y, s)-i_{u}(\hat{X}, \hat{t})
$$

takes its minimum at $(\hat{Y}, \hat{s})$. By Remark 3.2 (i) we see that

$$
-\partial_{s} \Xi(\hat{X}, \hat{t}, \hat{Y}, \hat{s}) \geq 0 .
$$

By (4.3) the inequality (4.5) is $2 \alpha(\hat{t}-\hat{s})+\sigma(T-\hat{t})^{-2} \leq 0$ and $(4.7)$ is $2 \alpha(\hat{t}-\hat{s}) \geq 0$. Combining these inequalities we obtain $\sigma>0$ which is a contradiction. So the Case 1 does not occur. 
We shall study the Case 2. This case is divided into two subcases.

Case 2A. For sufficiently large $\alpha$

$$
\partial_{z} \Xi(\hat{X}, \hat{t}, \hat{Y}, \hat{s}) \neq 0
$$

where $z$ is $n+1$ component of $X=(x, z)$.

Case 2B. There is a sequence $\alpha_{j}$ tending to infinity as $j \rightarrow \infty$ such that

$$
\partial_{z} \Xi(\hat{X}, \hat{t}, \hat{Y}, \hat{s})=0
$$

with $\hat{X}=\hat{X}\left(\alpha_{j}\right), \ldots$

In the Case 2A we apply Lemma 3.3 (i) for the function defined in (4.4) with $\Phi(x, t)=\Xi(X, t, \hat{Y}, \hat{s})-I_{v}(\hat{Y}, \hat{s})$ to get

$$
\tau+H(\hat{z}, \hat{p}) \leq 0
$$

with

$$
\begin{aligned}
& \tau=-\left(2 \alpha(\hat{t}-\hat{s})+\sigma(T-t)^{-2}\right) /(2 \alpha(\hat{z}-\hat{w})) \\
& p=-2 \alpha(\hat{x}-\hat{y}) / 2 \alpha(\hat{z}-\hat{w})
\end{aligned}
$$

by (4.3), where $\hat{X}=(\hat{x}, \hat{z}), \hat{Y}=(\hat{y}, \hat{w})$. Similarly, for the function defined in (4.6) we apply Remark 3.4 to get

$$
\tilde{\tau}+H(\hat{w}, \hat{p}) \geq 0
$$

with $\tilde{\tau}=-\partial_{s} \Xi / \partial_{w} \Xi=-2 \alpha(\hat{t}-\hat{s}) / 2 \alpha(\hat{z}-\hat{w})$. Since $\hat{z}<\hat{w},(4.8)$ and (4.9) yields

$$
\begin{gathered}
-2 \alpha(\hat{t}-\hat{s})+\sigma(T-t)^{-2}+2 \alpha(\hat{w}-\hat{z}) H(\hat{z}, \hat{p}) \leq 0 \\
-2 \alpha(\hat{t}-\hat{s})+2 \alpha(\hat{w}-\hat{z}) H(\hat{w}, \hat{p}) \geq 0 .
\end{gathered}
$$

respectively. Subtracting (4.11) from (4.10) and noting $\sigma(T-t)^{-2} \geq \sigma T^{-2}$ we obtain

$$
\sigma T^{-2}+2 \alpha(\hat{w}-\hat{z})(H(\hat{z}, \hat{p})-H(\hat{w}, \hat{p})) \leq 0 .
$$

By (H2) we see that

$$
\begin{aligned}
& |2 \alpha(\hat{w}-\hat{z})(H(\hat{z}, \hat{p})-H(\hat{w}, \hat{p}))| \\
& \leq 2 C \alpha|\hat{w}-\hat{z}|^{2}\left(1+\frac{|\hat{x}-\hat{y}|}{|\hat{w}-\hat{z}|}\right) \leq 4 C \alpha|\hat{X}-\hat{Y}|^{2} .
\end{aligned}
$$


Since (4.2) holds, letting $\alpha \rightarrow \infty$ in (4.12) yields $\sigma^{T^{-2}} \leq 0$ which is a contradiction.

It remains to discuss the Case $2 \mathrm{~B}$ which essentially relates to our definitions of proper viscosity solutions with shocks. For $\hat{X}=(\hat{x}, \hat{z}), \hat{Y}=(\hat{y}, \hat{w})$ by the assumption $\partial_{z} \Xi(\hat{X}, \hat{t}, \hat{Y}, \hat{s})=0, \hat{z}$ must agree with $\hat{w}$ and by Lemma $3.3, \hat{z} \leq u(\hat{x}, \hat{t}), \hat{w} \geq v(\hat{y}, \hat{s})$ so that $v(\hat{y}, \hat{s}) \leq u(\hat{x}, \hat{t})$. We next note that

$$
a_{0}=(\hat{x}, \hat{v}, \hat{t}, \hat{y}, \hat{v}, \hat{s}) \quad \text { and } a_{1}=(\hat{x}, \hat{u}, \hat{t}, \hat{y}, \hat{u}, \hat{s}) \quad \text { with } \quad \hat{u}=u(\hat{x}, \hat{t}), \hat{v}=v(\hat{y}, \hat{s})
$$

are also maximizers of $\Psi$. Indeed, since $i_{u}(\hat{x}, z, \hat{t})=0$ for $z \leq \hat{u}$ and $I_{v}(\hat{y}, w, \hat{s})=0$ for $w \geq \hat{v}, \Psi$ must take the same value for all $z, w \in \mathbf{R}$ satisfying $z \leq \hat{u}, w \geq \hat{v}$ and $z=w$. In particular $a_{0}$ and $a_{1}$ are maximizers of $\Psi$ since $\hat{v} \leq \hat{u}$.

Since $\Psi$ is maximized at $a_{0}$, we apply Lemma 3.3 (ii), (iii) for the function (4.4) (with $\hat{Y}=(\hat{y}, \hat{v}))$ to get

$$
\partial_{t} \Xi+H^{I}\left(\hat{u}, \nabla_{x} \Xi\right) \leq 0 \text { at } a_{0}
$$

with $I=[\hat{v}, \hat{u}]$. Similarly, we apply Remark 3.4 to the function (4.6) (with $\hat{X}=$ $(\hat{x}, \hat{u}))$ to get

$$
-\partial_{s} \Xi+H^{I}\left(\hat{v},-\nabla_{y} \Xi\right) \geq 0 \text { at } a_{1}
$$

Since

$$
\begin{aligned}
& \nabla_{x} \Xi\left(a_{0}\right)=2 \alpha(\hat{x}-\hat{y})=-\nabla_{y} \Xi\left(a_{1}\right), \\
& \partial_{t} \Xi\left(a_{0}\right)=\sigma(T-t)^{-2}+2 \alpha(\hat{t}-\hat{s}), \\
& -\partial_{s} \Xi\left(a_{1}\right)=2 \alpha(\hat{t}-\hat{s}),
\end{aligned}
$$

by (4.3) and since $H^{I}(r, p)$ is nondecreasing in $r$, subtracting (4.14) for (4.13) yields

$$
\sigma(T-\hat{t})^{-2} \leq 0
$$

This yields contradiction $\sigma \leq 0$. We have thus proved that Case $2 \mathrm{~B}$ does not occur. Thus in any cases we get a contradiction which proves $u<v$ in $\Omega \times(0, T)$.

Remark 4.2. (i) We do not intend to prove the weak comparison principle as general as possible. However, we note that above proof does work for the case when $H$ also depends on $x$ provided that

$$
\left|H(x, r, p)-H\left(x^{\prime}, r^{\prime}, p\right)\right| \leq C^{\gamma}\left(\left|r-r^{\prime}\right|+\left|x-x^{\prime}\right|\right)(1+|p|)
$$


instead of (H2).

(ii) In general $f^{\prime} \leq g^{\prime}$ does not imply $f_{I}^{\prime} \leq g_{I}^{\prime}$. This indicates that a proper supersolution $v$ of (2.1) in $Q=\Omega \times(0, T)$ may not be a proper supersolution of

$$
\partial_{t} u+\tilde{H}(x, t, u, \nabla u)=0
$$

in $Q$ for $\tilde{H} \geq H$. However, if one of $f^{\prime}$ and $g^{\prime}$ is a constant function, then $f^{\prime} \leq g^{\prime}$ implies $f_{I}^{\prime} \leq g_{I}^{\prime}$. Indeed, we may assume that $g^{\prime}$ is a constant $c$ since the proof for the other case is symmetric. We may also assume that $c=0$ by considering $f(r)-c r$ instead of $f$ since $(f-c r)_{I}=f_{I}-c r$. If $f^{\prime} \leq 0$, then $f_{I}$ must be nonincreasing so that $f_{I}^{\prime} \leq 0$. Thus $f^{\prime} \leq g^{\prime}$ implies $f_{I}^{\prime} \leq g_{I}^{\prime}$ when one of $f^{\prime}$ and $g^{\prime}$ is a constant. This property yields the next lemma.

Lemma 4.3. Assume that one of $H(x, t, r, p), \tilde{H}(x, t, r, p)$ is independent of $r$ and that $H \leq \tilde{H}$. If $u$ is a proper subsolution of (4.15) (resp. supersolution of (2.1)) in $Q$, then it is a proper subsolution of (2.1) (resp. supersolution of (4.15)) in $Q$.

\section{Proper comparison principle}

The weak comparison principle WCP (Theorem 4.1) is unfortunately too weak to guarantee the uniqueness of solution. This phenomenon is related to fattening in geometric equations like curvature flow equations. As shown in [5] a solution of

$$
\partial_{t} u+(x-t)\left|\partial_{x} u\right|=0
$$

starting with a characteristic function $\chi_{I}$ of some closed interval $I$ is not unique. In fact they proved that for continuous initial data $u_{0}$ with $I=\left\{u_{0} \geq 0\right\}$ and int $I=\left\{u_{0}>0\right\}$, the zero level set of solution $u$ with initial data $u_{0}$ may have interior for $t>0$ so that solutions with initial data $\chi_{I}$ is not unique. Formation of interior for the level set is called fattening. By Proposition 2.4 for (5.1) a usual sub- and supersolution are also a proper sub- and supersolution, respectively. Our statement of WCP does not include (5.1) since $H$ depends on $x$ and $t$. However, the proof applies to (5.1) also, so WCP holds for (5.1), although the solution is not unique.

\subsection{Strictly monotone evolutions}

In some cases such situation does not occur. A typical situation is that the evolution is monotone in time. Here are typical assumptions. 
(H4a) $-H(r, p) \geq \rho \sqrt{1+|p|^{2}}$ with some $\rho>0$ for all $p \in \mathbf{R}^{n}, r \in \mathbf{R}$.

(H4b) $H(r, p) \geq \rho \sqrt{1+|p|^{2}}$ with some $\rho>0$ for all $p \in \mathbf{R}^{n}, r \in \mathbf{R}$.

Theorem 5.1 (Proper comparison principle). Assume (H1)-(H3). Assume (H4a) or (H4b). Let $u$ and $v$ be bounded proper sub- and supersolutions of (4.1) in $\mathbf{T}^{n} \times(0, T)$, respectively. Assume that

$$
u^{*}(x, 0) \leq\left(v_{*}\right)^{*}(x, 0) \quad\left(\operatorname{resp} .\left(u^{*}\right)_{*}(x, 0) \leq v_{*}(x, 0)\right) \quad \text { for all } x \in \mathbf{T}^{n} .
$$

Then

$$
u^{*} \leq\left(v_{*}\right)^{*} \quad\left(\text { resp. }\left(u^{*}\right)_{*} \leq v_{*}\right) \quad \text { in } \mathbf{T}^{n} \times[0, T) .
$$

Remark 5.2. (i) We give a geometric interpretation of Theorem 5.1. The assumption (5.2) on initial data implies

$$
\operatorname{int}(\overline{\operatorname{sg} u}(0) \cap \overline{\operatorname{Sg} v}(0))=\emptyset,
$$

where $E(t)$ denotes the cross-section at $t$ of a subset $E$ of $\mathbf{T}^{n} \times \mathbf{R} \times[0, T]$ i.e. $E(t)=\left\{(x, z) \in \mathbf{T}^{n} \times \mathbf{R} ;(x, z, t) \in E\right\}$. The condition (5.3) implies

$$
\operatorname{int}(\overline{\operatorname{sg} u} \cap \overline{\operatorname{Sg} v})=\emptyset \text {. }
$$

(ii) In CP the assumption on initial data is $u^{*} \leq v_{*}$ at $t=0$ is stronger than (5.2); in CP the conclusion is also stronger than that of Theorem 5.1. Since the comparison principle in Theorem 5.1 is not comparable both CP and WCP, we refer Theorem 5.1 as a proper comparison principle.

Proof. We may assume that $u$ and $-v$ are upper semicontinuous in $\mathbf{T}^{n} \times[0, T)$. We first assume (H4a). By (H4a) and Lemma $4.3 v$ is a proper supersolution of

$$
\partial_{t} w-\rho \sqrt{1+|\nabla w|^{2}}=0 .
$$

Formally, if $w$ solves (5.4), the set $\mathrm{sg} w$ moves by $V=\rho$, where $V$ is the outward normal velocity of $\partial(\mathrm{sg} u(t))$ in $\mathbf{T}^{n} \times \mathbf{R}$. It is well-known (e.g. [5]) that the equation (5.4) does not have fattening phenomena. In other words we have the following containment principle: if $\operatorname{sg} w_{1}$ and sg $w_{2}$ are sub- and supersolution of the surface evolution equation $V=\rho$ as sets (see e.g. [38]) and initially sg $w_{1}(0) \subset \overline{\operatorname{sg} w_{2}}(0$ ), 
then sg $w_{1} \subset \overline{\operatorname{sg} w_{2}}$. Since our $v$ is a supersolution of (5.4) so that $I_{v}$ is a supersolution of the level set equation [23] of $V=\rho, \operatorname{sg} v$ is a supersolution of $V=\rho$. Since

$$
E=\left\{(x, z, t) \in \mathbf{T}^{n} \times \mathbf{R} \times[0, T), \operatorname{dist}(x, \overline{\operatorname{sg} v}(0)) \leq \rho t\right\}
$$

is a solution of $V=\rho$, the containment principle yields $E \subset \overline{\operatorname{sg} v}$. In particular, for $\delta \in(0, T)$ there is $\rho^{\prime}>0$ that satisfies

$$
v(x, t) \geq v^{*}(x, 0)+\rho^{\prime} \quad \text { for all } x \in \mathbf{T}^{n}, t \geq \delta .
$$

We shift $v$ in time and set

$$
v_{\delta}(x, t)=v(x, t+\delta), \quad t>0
$$

Evidently, $v_{\delta}$ is a proper supersolution of $(4.1)$ in $\mathbf{T}^{n} \times(0, T-\delta)$. Assume that $u \leq v^{*}$ at $t=0$. By (5.5) we see

$$
u \leq v^{*} \leq v_{\delta}-\rho^{\prime} \quad \text { at } t=0
$$

Since $v_{\delta}$ is lower semicontinuous up to $t=0$, applying WCP (Theorem 4.1) we get $u \leq v_{\delta}$ in $\mathbf{T}^{n} \times[0, T)$. Sending $\delta$ to zero we conclude that $u \leq v^{*}$ in $\mathbf{T}^{n} \times[0, T)$ since $\varlimsup_{\lim _{\delta \rightarrow 0}} v_{\delta} \leq v^{*}$ by the definition of upper semicontinuous envelope.

If we assume $u_{*} \leq v$ at $t=0$, then $\left(u_{*}\right)^{*}<v_{\delta}$ at $t=0$. By WCP we have $\left(u_{*}\right)^{*} \leq v_{\delta}$ in $\mathbf{T}^{n} \times[0, T)$. By definition $v \geq \liminf _{* \delta \rightarrow 0} v_{\delta}$ in $\mathbf{T}^{n} \times(0, T)$, where $\liminf _{*}$ denotes the relaxed limit defined right after Theorem 6.1. We take liminf $*$ for $u_{*}=\left(\left(u_{*}\right)^{*}\right)_{*} \leq v_{\delta}$ to get $u_{*} \leq v$ in $\mathbf{T}^{n} \times(0, T)$; the last inequality holds also at $t=0$ by the assumption.

The proof under $(\mathrm{H} 4 \mathrm{~b})$ is symmetric. We shift $u$ instead of $v$ in this case. We omit the detail.

Definition 5.3. We say that $u: \mathbf{T}^{n} \times(0, T) \rightarrow \mathbf{R} \cup\{ \pm \infty\}$ is a solution of (4.1) with initial data $u_{0}$ if $u$ is a proper solution of $(4.1)$ in $\mathbf{T}^{n} \times(0, T)$ and

$$
u^{*}(x, 0)=\left(u_{*}\right)^{*}(x, 0)=u_{0}^{*}(x), \quad u_{*}(x, 0)=\left(u^{*}\right)_{*}(x, 0)=u_{0 *}(x),
$$

where $u_{0}: \mathbf{T}^{n} \rightarrow \mathbf{R} \cup\{ \pm \infty\}$

Theorem 5.4 (Uniqueness of solution). Assume (H1)-(H3). Assume (H4a) or (H4b). Let $u$ be a bounded proper solution of (4.1) with initial data $u_{0}$. Then $u^{*}$ and $u_{*}$ are unique and moreover, $\left(u_{*}\right)^{*}=u^{*}$ and $\left(u^{*}\right)_{*}=u_{*}$. 
Proof. Assume (H4a). Let $\bar{u}$ be another solution. Since $u^{*} \leq\left(\bar{u}_{*}\right)^{*}$ at $t=0$, the proper comparison principle (Theorem 5.1) implies that $u^{*} \leq\left(\bar{u}_{*}\right)^{*}\left(\leq \bar{u}^{*}\right)$ in $\mathbf{T}^{n} \times(0, T)$. Replacing the role of $\bar{u}$ and $u$ yields $\bar{u}^{*} \leq u^{*}$. We thus conclude that $\bar{u}^{*}=u^{*}$. Moreover, $u^{*} \leq\left(u_{*}\right)^{*} \leq u^{*}$ implies $\left(u_{*}\right)^{*}=u^{*}$. A symmetric argument implies the uniqueness of $u_{*}$ and $\left(u^{*}\right)_{*}=u_{*}$. The proof for $(\mathrm{H} 4 \mathrm{~b})$ is the same.

Remark 5.5. (i) If a proper solution $u$ for initial data $u_{0}$ exists when (H4a) or (H4b) holds, then $u_{0}$ must satisfy $\left(u_{0 *}\right)^{*}=u_{0}^{*}$ and $\left(u_{0}^{*}\right)_{*}=u_{0 *}$. Even if $u_{0}$ is upper semicontinuous, the solution in Definition 5.3 does not exist unless $\left(u_{0 *}\right)^{*}=u_{0}$ when (H4a) holds. So to discuss solvability we must assume $\left(u_{0 *}\right)^{*}=u_{0}$ for initial data under (H4a).

(ii) Topological operations of subgraphs and supergraphs relate envelope operations of functions. For example

$$
\operatorname{int}(\operatorname{sg} u)=\left(\operatorname{Sg} u_{*}\right)^{c}, \overline{(\operatorname{Sg} u)^{c}}=\operatorname{sg} u^{*} .
$$

Thus the condition $\left(u_{*}\right)^{*}=u$ is equivalent to $\overline{\operatorname{int}(\operatorname{sg} u)}=\operatorname{sg} u$. Here $A^{c}$ denotes the complement set of $A$.

\subsection{Monotone evolutions for homogeneous equations}

We now weaken the assumption (H4a) to handle the equation (1.6). Instead of (H4a), (H4b) we consider one of following situation

$$
-H(r, p) \geq \rho|p| \text { with some } \rho>0 \text { for all } p \in \mathbf{R}^{n}, r \in \mathbf{R} \text {. }
$$

$$
H(r, p) \geq \rho|p| \text { with some } \rho>0 \text { for all } p \in \mathbf{R}^{n}, r \in \mathbf{R} \text {. }
$$

Unfortunately, we are unable to prove results of Theorem 5.1 in this generality. We restrict ourselves when (4.1) is homogeneous in the sense that $p \mapsto H(r, p)$ is positively homogeneous of degree one. A general form of a homogeneous equation is of the form (1.6). Still there seems to be technical difficulty to prove the proper comparison principle for homogeneous equations (4.1) for general initial data. A major reason is that the motion of $\mathrm{sg} u$ of (4.1) is not strictly monotone increasing in the vertical direction so the relation (5.5) is not expected. To overcome this difficulty we consider a special class of initial data.

For $w: \mathbf{T}^{n} \rightarrow \mathbf{R} \cup\{ \pm \infty\}$ we say that $w$ satisfies no plateau condition if $E_{c}=$ $\bar{D}_{c}$ for all $c \in\left(\inf w_{*}, \sup w^{*}\right)$ with $E_{c}=\left\{x \in \mathbf{T}^{n} ; w^{*}(x) \geq c\right\}$ and $D_{c}=\{x \in$ 
$\left.\mathrm{T}^{n}, w_{*}(x)>c\right\}$. Here $\bar{A}$ denotes the closure of $A$. The set $E_{c}$ is closed while $D_{c}$ is open and $D_{c} \subset E_{c}$. This no plateau condition is stronger than $\left(w_{*}\right)^{*}=w^{*}$ and $\left(w^{*}\right)_{*}=w_{*}$. Also it is easy to see that $w$ fulfills no plateau condition if and only if $w_{*}$ (or $w^{*}$ ) fulfill the condition. If $\{w=c\}$ has an interior for some $c \in\left(\inf w_{*}, \sup w^{*}\right)$, $w$ should not fulfill no plateau condition since a 'plateau' exists. The next lemma easily follows from definition. For a set $A$ in $\mathbf{T}^{n} \times \mathbf{R}(\times[0, T))$ we set $\gamma_{m M} A=$ $A \cap \mathbf{T}^{n} \times(m, M)(\times[0, T))$.

Lemma 5.6. Assume that $v$ satisfies no plateau condition and bounded. For $\sigma>0$ let $w_{\sigma}$ be

$$
w_{\sigma}(x)=\sup \{v(y) ;|y-x| \leq \sigma\}
$$

Then

$$
\overline{\gamma_{m M} \mathrm{Sg} w_{\sigma}} \cap \overline{\gamma_{m M} \mathrm{sg} v^{*}}=\emptyset
$$

as a set in $\mathbf{T}^{n} \times[m, M]$, where $m=\min v_{*}, M=\max v^{*}$.

For homogeneous equations we are able to prove a weak comparison principle under weaker assumptions. Since the proof parallels that of WCP we only give its sketch.

Theorem 5.7. Assume (H1), (H2). Assume that $H$ is positively homogeneous of degree one in $p$ (so that (4.1) is homogeneous). Let $u$ and $v$ be bounded proper sub- and supersolution of (4.1) in $\mathrm{T}^{n} \times(0, T)$, respectively. Assume that

$$
\overline{\gamma_{m M} \operatorname{Sg}\left(\left.v_{*}\right|_{t=0}\right)} \cap \overline{\gamma_{m M} \operatorname{sg}\left(\left.u^{*}\right|_{t=0}\right)}=\emptyset
$$

as sets in $\mathbf{T}^{n} \times[m, M]$ with $m=\min _{x} v_{*}(x, 0), M=\max _{x} u^{*}(x, 0)$. Then

$$
\overline{\gamma_{m M} \mathrm{Sg} v_{*}} \cap \overline{\gamma_{m M} \mathrm{Sg} u^{*}}=\emptyset
$$

as sets in $\mathbf{T}^{n} \times[m, M] \times[0, T)$. In particular $u^{*} \leq\left(v_{*}\right)^{*}$ in $\mathbf{T}^{n} \times[0, T)$.

The same assertion is still valid if $\mathbf{T}^{n}$ is replaced by $\mathbf{R}^{n}$ provided that $\overline{\gamma_{m M} \operatorname{Sg} v_{*}} \cap$ $\overline{\gamma_{m M} \mathrm{sg} u_{*}}$ is bounded.

Sketch of the proof. Since a constant function is always a proper solution for homogeneous equation, by WCP we see that $u^{*} \leq M$ and $v_{*} \geq m$ for all $t \in[0, T)$. It is easy to see that $v_{M}=\min (v, M)$ and $u_{m}=\max (u, m)$ are proper super- and subsolution, respectively and (5.7), (5.8) are unchanged under this modification. 
So we may assume that $m \leq u \leq M, m \leq v \leq M$ on $\mathbf{T}^{n} \times[0, T)$. We consider $\Psi(X, t, Y, s)$ as in the proof of Theorem 4.1 but only on $Z_{m M} \times Z_{m M}$ instead of $Z \times Z$ with $Z_{m M}=\mathbf{T}^{n} \times[m, M] \times[0, T]$ while $Z=\mathbf{T}^{n} \times \mathbf{R} \times[0, T]$. We argue in the same way in the proof of Theorem 4.1 to get the desired result (5.8). Here is one caution. When $\hat{z}, \hat{w}=m$ or $M$, Proposition 3.1 and Lemma 3.3 do not apply directly to derive (4.8), (4.9), (4.13), (4.14). Instead we have to apply Lemma 5.8 stated below.

The proof for $\mathbf{R}^{n}$ is essentially the same. We replace $\mathbf{T}^{n}$ by $B_{R}(0)$ satisfying

$$
\overline{\gamma_{m M} \mathrm{Sg} v_{*}} \cap \overline{\gamma_{m M} \mathrm{Sg} u^{*}} \subset\left(\text { int } B_{R}(0)\right) \times[m, M] \times[0, T) .
$$

and study a maximizer of $\Psi(X, t, Y, s)$.

Lemma 5.8. Let $u$ be a proper subsolution of a homogeneous equation (4.1) in $Q=\Omega \times(0, T)$. For $m=\inf _{Q} u^{*}$ let $v$ be a function defined by

$$
v(x, t)=u^{*}(x, t) \quad \text { if } u^{*}(x, t)>m ; v(x, t)=-\infty \quad \text { if } u^{*}(x, t)=m
$$

Let $\Phi$ be $C^{\infty}$ in $\hat{Q}_{m}=\Omega \times[m, \infty) \times(0, T)$. Assume that $(\hat{x}, m, \hat{t}) \in \hat{Q}_{m}$ is a maximizer of $i_{v^{*}}-\Phi$ in $\hat{Q}_{m}$.

(i) If $\hat{\nabla} \Phi(\hat{x}, m, \hat{t})=0$, then $\partial_{t} \Phi\left(x_{0}, z_{0}, t_{0}\right) \leq 0$.

(ii) If $\partial_{z} \Phi(\hat{x}, m, \hat{t}) \neq 0$, then $m=u^{*}(\hat{x}, \hat{t})$ and $\partial_{z} \Phi(\hat{x}, m, \hat{t})<0$. Moreover,

$$
\partial_{t} \Phi(\hat{x}, m, \hat{t})+H\left(m, \nabla_{x} \Phi(\hat{x}, m, \hat{t})\right) \leq 0 \text {. }
$$

(iii) If $\partial_{z} \Phi(\hat{x}, m, \hat{t})=0$ and $\nabla_{x} \Phi(\hat{x}, m, \hat{t}) \neq 0$, then

$$
\partial_{t} \Phi(\hat{x}, m, \hat{t})+H^{I}\left(m, \nabla_{x} \Phi(\hat{x}, m, \hat{t})\right) \leq 0 \quad \text { with } I=\left[m, u^{*}(\hat{x}, \hat{t})\right] .
$$

Proof. (i) As in the proof of Proposition 3.1 it suffices to prove that $v^{*}$ is right accessible at $(\hat{x}, \hat{t})$. If $v^{*}(\hat{x}, \hat{t})>m$, by definition $v^{*}(\hat{x}, \hat{t})=u^{*}(\hat{x}, \hat{t})(>m)$ and $v^{*}$ is right accessible at $(\hat{x}, \hat{t})$. If $v^{*}$ is not right accessible at $(\hat{x}, \hat{t})$ when $v^{*}(\hat{x}, \hat{t})=m$, $v^{*}(\hat{x}, \hat{t})=-\infty$ near $(\hat{x}, \hat{t})$ for $t<\hat{t}$. This would contradict "finite speed propagation" (e.g. [8]) of level sets of solutions of level set equations as we shall explain below. By definition of $v$

$$
u^{*}(x, t)=m \quad \text { for }(x, t) \in B_{r}(\hat{x}) \times[\hat{t}-\delta, \hat{t})
$$


for some small $\delta>0$ and $r>0$. We set

$$
\sigma:=\max \left\{u^{*}(x, t) ;(x, t) \in B_{r}(\hat{x}) \times[\hat{t}-\delta, \hat{t}+\delta]\right\}
$$

and observe that $\sigma$ is finite. By continuity and homogeneity of $H$

$$
\alpha:=\inf \left\{H(r, p) /|p| ; m-1 \leq r \leq \sigma+1, p \in \mathbf{R}^{n}\right\}>-\infty
$$

By Lemma 4.3 we see that $u$ is a subsolution of

$$
\partial_{t} u+\alpha|\nabla u|=0 \quad \text { in int } B_{r}(\hat{x}) \times(\hat{t}-\delta, \hat{t}+\delta) .
$$

Clearly for $r_{0} \in(0, r)$ the function

$$
w(x, t)= \begin{cases}m, & x \in B_{r_{0}+\alpha(t-\hat{t})}(\hat{x}), \\ \sigma+1, & \text { otherwise }\end{cases}
$$

is a supersolution of (5.9) in int $B_{r}(\hat{x}) \times\left(\hat{t}-\delta^{\prime}, \hat{t}+\delta^{\prime}\right)$ for small $\delta^{\prime}<\delta$. By CP for (5.9) (see e.g. [8]) $u^{*} \leq w$ near $(\hat{x}, \hat{t})$. This implies that $u^{*}(x, t)=m$ in a neighborhood of $(\hat{x}, \hat{t})$ so $v^{*}(\hat{x}, \hat{t})=-\infty$. This is a contradiction so we have proved that $v^{*}$ is right accessible at $(\hat{x}, \hat{t})$ even if $v^{*}(\hat{x}, \hat{t})=m$.

(ii) The properties $m=v^{*}(\hat{x}, \hat{t})$ and $\partial_{z} \Phi(\hat{x}, m, \hat{t})<0$ are easy to prove. We may assume that there is no maximum of $i_{v^{*}}-\Phi$ other than $(\hat{x}, m, \hat{t})$ in $\hat{Q}_{m}$. We set

$$
\Phi_{\varepsilon}(x, z, t)=\Phi(x, z, t)+\varepsilon /(z-m) .
$$

By convergence of maximum points (e.g. [4], [17]) there is a sequence $\left(x_{\varepsilon}, z_{\varepsilon}, t_{\varepsilon}\right) \rightarrow$ $(\hat{x}, m, \hat{t})(\varepsilon \rightarrow 0)$ satisfying

$$
\max _{\hat{Q}_{m}}\left(i_{v^{*}}-\Phi_{\varepsilon}\right)=\left(i_{v^{*}}-\Phi_{\varepsilon}\right)\left(x_{\varepsilon}, z_{\varepsilon}, t_{\varepsilon}\right)
$$

Since $\partial_{z} \Phi_{\varepsilon}\left(x_{\varepsilon}, z_{\varepsilon}, t_{\varepsilon}\right)<0$ for small $\varepsilon>0$ and $z_{\varepsilon}>m$, Lemma 3.3 (i) yields

$$
\partial_{t} \Phi\left(x_{\varepsilon}, z_{\varepsilon}, t_{\varepsilon}\right)+H\left(z_{\varepsilon}, \nabla_{x} \Phi\left(x_{\varepsilon}, z_{\varepsilon}, t_{\varepsilon}\right)\right) \leq 0
$$

by the homogeneity of $H$. Sending $\varepsilon \rightarrow 0$ yields the desired inequality.

(iii) Since the proof is essentially the same as that of Lemma 3.3 (ii), (iii) we do not repeat it here.

We are now in position to state a kind of proper comparison principle for homogeneous equation under ( $\mathrm{H} 5 \mathrm{a})$. Since the statement is symmetric when $(\mathrm{H} 5 \mathrm{~b})$ is assumed, we do not present the version under $(\mathrm{H} 5 \mathrm{~b})$. 
Theorem 5.9. Assume (H1), (H2), (H5a). Assume that $H(r, p)$ is positively homogeneous of degree one in $p$ (so that (4.1) is homogeneous). Let $u$ and $v$ be bounded proper sub- and supersolutions of (4.1) in $\mathrm{T}^{n} \times(0, T)$, respectively. Assume that

$$
u^{*}(x, 0) \leq\left(v_{*}\right)^{*}(x, 0) \quad\left(\text { resp. } \quad\left(u^{*}\right)_{*}(x, 0) \leq v_{*}(x, 0)\right) \quad \text { for all } x \in \mathbf{T}^{n}
$$

If $v_{*}(x, 0)$ fulfills no plateau condition as a function of $x$, then

$$
u^{*} \leq\left(v_{*}\right)^{*} \quad\left(\operatorname{resp} . \quad\left(u^{*}\right)_{*} \leq v_{*}\right) \quad \text { in } \mathbf{T}^{n} \times[0, T) .
$$

Proof. We may assume that $u$ and $-v$ are upper semicontinuous in $\mathbf{T}^{n} \times[0, T)$. As in the sketch of the proof of Theorem 5.7 we may assume that

$$
\begin{aligned}
& m \leq u \leq M, m \leq v \leq M \text { on } \mathbf{T}^{n} \times[0, T) \text { for } m=\min _{x} v(x, 0), \\
& M=\max _{x} u(x, 0) .
\end{aligned}
$$

By (H5a) and Lemma $4.3 v$ is a proper supersolution of

$$
\partial_{t} W-\rho|\nabla W|=0
$$

The viscosity solution of (5.10) with initial data $v(x, 0)$ is given by

$$
W(x, t)=\sup \{v(y, 0) ;|x-y| \leq \rho t\}
$$

(which is a special case of the Hopf formula [3].) This formula is easy to guess since each level set of solution $W$ of (5.10) moves its normal velocity $\rho$ at least formally. This equation (5.10) does not have fattening phenomena (e.g. [5]) so by containtment principle we have

$$
W(x, t) \leq v^{*}(x, t) \text { for } t \geq 0
$$

Since sg $W$ only grows in the horizontal direction $x$, this unfortunately does not imply (5.5). However, thanks to the relation (5.6) in Lemma 5.6 we observe from (5.11) that

$$
\overline{\gamma_{m M} \operatorname{Sg} v(t)} \cap \overline{\gamma_{m M} \operatorname{sg} u(0)}=\emptyset \text { for all } t \in(0, T)
$$

if we assume that $u \leq v^{*}$ at $t=0$. 
We shift $v$ in time and set

$$
v_{\delta}(x, t)=v(x, t+\delta)
$$

By definition $v_{\delta}$ is a proper supersolution of (4.1) in $\mathbf{T}^{n} \times(0, T-\delta)$. Since (5.12) implies (5.7) with $v=v_{\delta}$, we apply Theorem 5.7 to get $u \leq v_{\delta}$ on $\mathbf{T}^{n} \times[0, T)$. Sending $\delta$ to zero we conclude that $u \leq v^{*}$ in $\mathbf{T}^{n} \times[0, T)$ since $\varlimsup_{\delta \rightarrow 0} v_{\delta} \leq v^{*}$. The proof under the assumption $u_{*} \leq v$ at $t=0$ is similar (see the proof of Theorem $5.1)$.

Remark 5.10. From Theorem 5.9 it follows the uniqueness of a proper solution corresponding to Theorem 5.4.

\subsection{Homogeneous equations with monotone data}

We give another type of proper comparison principle. Instead of assuming monotonicity in time for evolutions we assume some monotonicity on initial data.

Let $v_{0}$ be a bounded function on $\mathbf{R}^{n}$. We say that $v_{0}$ is strictly spokewise decreasing if

$$
\begin{gathered}
\overline{\gamma_{m M} \operatorname{Sg}\left(D_{\lambda}^{0} v_{0 *}\right)} \cap \overline{\gamma_{m M} \operatorname{sg} v_{0}^{*}}=\emptyset, \\
M=\sup v_{0}^{*}, \quad m=\inf v_{0 *} \quad \text { for } \lambda \in(0,1),
\end{gathered}
$$

where $\left(D_{\lambda}^{0} f\right)(x)=f(\lambda x)$. Here the closure is taken in $\mathbf{R}^{n} \times[m, M]$. It is easy to see that (5.13) implies that $s \mapsto v\left(s x_{0}\right)\left(x_{0} \in \mathbf{R}^{*}, x_{0} \neq 0\right)$ is strictly decreasing in $s \in[0, \infty)$ unless $v\left(s x_{0}\right)=m$ or $M$. Moreover, $v$ fulfills no plateau condition. The condition (5.13) is a kind of star-shapedness condition for level set of $v$. As pointed out in [38] and [5] 'star-shapedness' prevents fattening for level set equations, so we expect that a kind of proper comparison principle still holds for strictly spokewise decreasing initial data. We give a version of the proper comparison principle. Although a symmetric argument holds for strictly spokewise increasing initial data if it is properly defined, we restrict ourselves into decreasing initial data for simplicity of presentation.

Theorem 5.11. Assume (H1), (H2). Assume that (4.1) is homogeneous. Let $u$ and $v$ be a bounded proper sub-and supersolutions of (4.1) in $\mathbf{R}^{n} \times(0, T)$. Assume that

$$
v(x, t) \geq \inf _{\mathbf{R}^{n}} v_{*}(\cdot, 0) \geq u(x, t) \quad \text { for } \quad(x, t) \in\left(\mathbf{R}^{n} \backslash B_{R}(0)\right) \times(0, T)
$$


for some $R>0$. Assume that

$$
u^{*}(x, 0) \leq\left(v_{*}\right)^{*}(x, 0) \quad\left(\text { resp. } \quad\left(u^{*}\right)_{*}(x, 0) \leq v_{*}(x, 0)\right) \quad \text { for all } x \in \mathbf{R}^{n}
$$

If $v_{*}(\cdot, 0)$ is strictly spokewise decreasing, then

$$
u^{*} \leq\left(v_{*}\right)^{*} \quad\left(\operatorname{resp} . \quad\left(u^{*}\right)_{*} \leq v_{*}\right) \text { in } \mathbf{R}^{n} \times[0, T)
$$

Proof. As in the proof of Theorem 5.9 we may assume that $u$ and $-v$ are upper semicontinuous in $\mathbf{R}^{n} \times[0, T)$ and

$$
\begin{aligned}
& m \leq u \leq M, \quad m \leq v \leq M \text { on } \mathbf{R}^{n} \times[0, T) \\
& \text { for } m=\min _{x} v(x, 0), \quad M=\max _{x}(x, 0) .
\end{aligned}
$$

Since we have assumed that (4.1) is homogeneous,

$$
v_{\lambda}(x, t)=v(\lambda x, \lambda t), \quad \lambda>0
$$

is also a proper supersolution of $(4.1)$. Since $v(\cdot, 0)$ is strictly spokewise decreasing, it follows from (5.13) that (5.7) holds for $v=v_{\lambda}$ with $\lambda \in(0,1)$ if we assume that $u(x, 0) \leq v^{*}(x, 0)$.

By (5.13) we are able to apply a weak comparison principle (Theorem 5.7) in the case of $\mathbf{R}^{n}$ and obtain that $u \leq v_{\lambda *}$ on $\mathbf{R}^{n} \times[0, T)$. Sending $\lambda \in(0,1)$ to 1 , we conclude that $u \leq v^{*}$. A modification similar to that of Theorem 5.1 yields $u_{*} \leq v$ if $u_{*} \leq v$ at $t=0$ is assumed.

Remark 5.12. In one-dimensional case $(n=1)$ the condition (5.14) can be weakened as

$$
\begin{aligned}
& v \geq \inf _{\mathbf{R}} v_{*}(\cdot, 0) \geq u \quad \text { on }(R, \infty) \times(0, T), \\
& v \geq \sup _{\mathbf{R}} u^{*}(\cdot, 0) \geq u \text { on }(-\infty,-R) \times(0, T) .
\end{aligned}
$$

This condition is useful to handle monotone decreasing initial data.

From the proper comparison principle (Theorem 5.11) we have a kind of uniqueness of a proper solution like Theorem 5.4. We shall state the uniqueness whose proof is essentially the same as that of Theorem 5.4. 
Definition 5.13. We say that $u: \mathbf{R}^{n} \times(0, \infty) \rightarrow \mathbf{R} \cup\{ \pm \infty\}$ is a solution of a homogeneous equation (4.1) with initial data $u_{0}$ if $u$ is a proper solution of $\mathbf{R}^{n} \times$ $(0, \infty)$ satisfying $u^{*}(x, 0)=\left(u_{*}\right)^{*}(x, 0)=u_{0}^{*}(x), u_{*}(x, 0)=\left(u^{*}\right)_{*}(x, 0)=u_{0 *}(x)$ and moreover for any $T>0, u$ is locally constant and continuous in $\left(\mathbf{R}^{n} \backslash B_{R}(0)\right) \times[0, T]$ for some $R>0$. For $n \geq 2$ the last condition on $u$ is equivalent to that $u$ is a constant on $\left(\mathbf{R}^{n} \backslash B_{R}(0)\right) \times[0, T]$. However, when $n=1$ this allows the possibility that $u=\alpha$ on $(R, \infty) \times[0, T]$ and $u=\beta$ on $(-\infty,-R) \times[0, T]$ and $\alpha, \beta$ are constants which may be different.

Theorem 5.14. Assume (H1) and (H2). Assume that (4.1) is homogeneous. Assume that $u_{0}$ is locally constant and continuous outside some ball and that $u_{0}$ is strictly spokewise decreasing. Let $u$ be a bounded proper solution of (4.1) with initial data $u_{0}$. Then $u^{*}$ and $u_{*}$ are unique and moreover, $\left(u_{*}\right)^{*}=u^{*}$ and $\left(u^{*}\right)_{*}=u_{*}$.

\section{Vanishing viscosity method}

For a given initial data $u_{0}$ we shall construct a proper solution of (4.1) by a vanishing viscosity method. We approximate (4.1) by a strictly parabolic problem

$$
\partial_{t} u+H(u, \nabla u)=\varepsilon \Delta u
$$

and solve (6.1) to get an approximate solution $u^{\varepsilon}$. We hope to get a solution $u$ as the limit of $u^{\varepsilon}$ as $\varepsilon \rightarrow 0$. If $r \mapsto H(r, p)$ is nondecreasing, this approach is standard in the theory of viscosity solutions [4], [3], [10] to get a continuous solution for continuous initial data. In our setting the solution may be discontinuous in a finite time even if initial data is smooth. Thus the standard theory of viscosity solutions does not apply. Since $H$ may not have divergence structure, the theory of conservation law does not apply neither.

Fortunately for proper solutions we still have a nice stability result although the proof is not standard.

Theorem 6.1 (Stability). Assume (H1) and (H3). For $\varepsilon>0$ let $u^{\varepsilon}$ be a viscosity subsolution (resp. supersolution) of (6.1) in $\Omega \times(0, T)$. Then $\bar{u}=\limsup _{\varepsilon \rightarrow 0}^{*} u^{\varepsilon}$ (resp. $\underline{u}=\liminf _{* \varepsilon \rightarrow 0} u^{\varepsilon}$ ) is a proper subsolution (supersolution of $(4.1)$ in $\Omega \times(0, T)$ provided that $\bar{u}<\infty(\operatorname{resp} . \underline{u}>-\infty)$ on $\bar{\Omega} \times[0, T]$, where $\Omega$ is a domain in $\mathbf{R}^{n}$ and $T>0$. 
Here lim sup* denotes the relaxed limit defined by

$\left(\limsup _{\varepsilon \rightarrow 0}^{*} u^{\varepsilon}\right)(x, t)=\lim _{\varepsilon \rightarrow 0} \sup \left\{u^{\varepsilon}(y, s) ;|y-x|<\delta,|t-s|<\delta, \varepsilon<\delta,(y, s) \in \Omega \times(0, T)\right\}$ and $\liminf _{*} u^{\varepsilon}=-\left(\lim \sup ^{*}\left(-u^{\varepsilon}\right)\right)$. (We often suppress $\varepsilon \rightarrow 0$ attached to limsup* and $\liminf$ in $_{*}$.

The result looks standard. However, we have to test surfaces for shocks other than usual test functions. We shall construct $\varepsilon$-family of test functions (viscous shocks) to approximate shocks. We shall give a full proof in $\S 6.1$.

In the next step we observe that there is no initial layer as $\varepsilon \rightarrow 0$.

Theorem 6.2. Assume that $H$ is continuous in $\mathbf{R} \times \mathbf{R}^{n}$. For $\varepsilon>0$ let $u^{\varepsilon}$ be a viscosity subsolution (resp. supersolution) of (6.1) in $\mathbf{T}^{n} \times(0, T)$ with $T>0$. For $u_{0}^{\varepsilon}(x)=u^{\varepsilon *}(x, 0)$ (resp. $u_{0}^{\varepsilon}(x)=u_{*}^{\varepsilon}(x, 0)$ ) let $u_{0}$ be the limit $u_{0}=\lim \sup _{\varepsilon \rightarrow 0}^{*} u_{0}^{\varepsilon}$ in (resp. $u_{0}=\liminf _{\varepsilon \rightarrow 0} u_{0}^{\varepsilon}$ ) as a function on $\mathbf{T}^{n}$. Assume that $\left\{u_{\varepsilon}\right\}$ is uniformly bounded on $\mathbf{T}^{n} \times[0, T)$ for small $\varepsilon$. Then $\bar{u}(x, 0) \leq u_{0}(x)$ (resp. $\underline{u}(x, 0) \geq u_{0}(x)$ ). (The same assertion is still valid if $\mathbf{T}^{n}$ is replaced by $\mathbf{R}^{n}$ ).

The proof is similar to the case when $r \mapsto H(r, p)$ is nondecreasing but we give it for completeness in $\S 6.1$.

Based on the stability result, Theorem 6.2 and the proper comparison principle we shall implement the vanishing viscosity method to construct a global proper solution of (4.1) for a given initial data. We state results on monotone evolutions with periodic data here and postpone to state the corresponding results on monotone data in $\S 6.3$.

We consider $(6.1)$ in $\mathbf{T}^{n} \times(0, T)$. We assume that

$$
\int_{1}^{\infty}-(H(r, 0))^{-1} d r=\infty, \int_{-\infty}^{-1} H(r, 0)^{-1} d r=\infty
$$

This condition prevents the blow up of solutions. It is equivalent to say that a globally spatially constant solution exists for every constant initial data. By comparison we see that (6.1) is globally solvable. We recall global existence of a solution to the approximate equation which is semilinear parabolic.

Proposition 6.3. Assume that $H(r, p)$ is Lipschitz in $p \in \mathbf{R}^{n}$ uniformly in $r \in[-R, R]$ for each $R>0$. Assume that (H2) and (6.2) hold. For $u_{0}^{\varepsilon} \in C\left(\mathrm{~T}^{n}\right)$ 
there exists a unique (classical) solution $u^{\varepsilon} \in C\left(\mathbf{T}^{n} \times[0, \infty)\right) \cap C^{2,1}\left(\mathbf{T}^{n} \times(0, \infty)\right)$ of (6.1) with $\left.u^{\varepsilon}\right|_{t=0}=u_{0}^{\varepsilon}$. Moreover, $F_{-}(t) \leq u^{\varepsilon}(x, t) \leq F_{+}(t)$ for $(x, t) \in \mathbf{T}^{n} \times(0, \infty)$, where $F_{+}$(resp. $F_{-}$) is a spatially constant global-in-time solution of (6.1) with initial data $\sup _{\mathbf{T}^{n}} u_{0}\left(\inf _{\mathbf{T}^{n}} u_{0}\right)$.

This result to standard and can be proved along the line of the proof of [36, Proposition 7.3.6] where the scalar conservation law is discussed. The unique local existence of solution is proved by a standard iteration argument. Its regularity is also standard. The assumption (6.2) guarantees the global a priori bound on $u$. Since the growth of $H(r, p)$ in $p$ is like $|p|$, this already yields a bound on $\nabla u$. Thus a local solution can be extended globally in time.

By Proposition 6.3 we are able to construct a proper solution as the limit of $u^{\varepsilon}$ as $\varepsilon \rightarrow 0$.

Theorem 6.4. Assume (H1)-(H3). Assume (H4a) or (H4b). Let $u_{0}$ be a bounded upper semicontinuous function on $\mathrm{T}^{n}$ with $\left(u_{0 *}\right)^{*}=u_{0}$.

(i) (Convergence). For $T \in(0, \infty)$ and $u_{0}^{\varepsilon} \in C\left(\mathbf{T}^{n}\right)(\varepsilon \in(0,1))$ let $u^{\varepsilon} \in C\left(\mathbf{T}^{n} \times\right.$ $[0, T))$ be a viscosity solution of (6.1) in $\mathbf{T}^{n} \times(0, T)$ with $\left.u^{\varepsilon}\right|_{t=0}=u_{0}^{\varepsilon}$. Assume that $\left\{u^{\varepsilon}\right\}$ is uniformly bounded on $\mathbf{T}^{n} \times\left[0, T^{\prime}\right)$ for small $\varepsilon$ for all $T^{\prime} \in(0, T)$. Assume that $u_{0}=\limsup _{\varepsilon \rightarrow 0}^{*} u_{0}^{\varepsilon}$ and $\left(u_{0}\right)_{*}=\liminf _{* \varepsilon \rightarrow 0} u_{0}^{\varepsilon}$. Then $\operatorname{sg} u^{\varepsilon}$ converges to sg $u$ in the sense of Hausdorff distance topology in $\mathbf{T}^{n} \times \mathbf{R} \times\left[0, T^{\prime}\right]$ for any $T^{\prime} \in(0, T)$ as $\varepsilon \rightarrow 0$ with some upper semicontinuous function $u$. The limit $u$ is a unique bounded (upper semicontinuous) proper solution of (4.1) in $\mathbf{T}^{n} \times\left(0, T^{\prime}\right)$ (for any $T^{\prime} \in(0, T)$ ) with initial data $u_{0}$.

(ii) (Existence). Assume (6.2). If $H(r, p)$ is Lipschitz in $p \in \mathbf{R}^{n}$ uniformly in $r \in[-R, R]$ for each $R>0$, then there exists a unique bounded proper (upper semicontinuous) solution $u$ of $(4.1)$ in $\mathbf{T}^{n} \times(0, T)$ with initial data $u_{0}$ for any $T \in$ $(0, \infty)$.

(If (4.1) is homogeneous, (H3) and (6.2) are unnecessary and (H5a) or (H5b) is assumed instead of ( $\mathrm{H} 4 \mathrm{a})$ or $(\mathrm{H} 4 \mathrm{~b})$. Moreover, the initial data $u_{0}$ is assumed to satisfy no plateau condition in the sense that $\left\{u_{0} \geq c\right\}=\overline{\left\{\left(u_{0}\right)_{*}>c\right\}}$ for all c except $c=\max u_{0}$ or $\min \left(u_{0}\right)_{*}$. The solution $u$ is bounded on $\mathbf{T}^{n} \times[0, \infty)$.)

Since $u_{0} \in C\left(\mathbf{T}^{n}\right)$ implies $\left(u_{0 *}\right)^{*}=u_{0}$ and (1.10) implies (6.2) and the local Lipschiz continuity implies (H1), (H2), (H3) and the regularity assumption on $H$ in (ii), Theorem 6.4 yields Theorems 1.1 and 1.3 for (1.6) and (1.7) under inf $a>0$, inf $b>0$. $\mathrm{t}$ As noted later (Remark 6.8) we also have the convergence of the supergraph 
$\operatorname{Sg} u^{\varepsilon} \rightarrow \operatorname{Sg} u$ and also graph $u^{\varepsilon} \rightarrow \operatorname{Sg} u_{*} \cap \operatorname{sg} u$. We postpone the proof of Theorem 6.4 (i) in $\S 6.2$. The part (ii) immediately follows from (i) and Proposition 6.3 since $F_{-} \leq u^{\varepsilon} \leq F_{+}$guarantees the uniform bound on $\left\{u^{\varepsilon}\right\}$.

Remark 6.5 (Size of discontinuities). Let $\Sigma$ denote the closure in $\mathbf{T}^{n} \times[0, \infty)$ of the set $\Sigma_{0}$ of jump discontinuities of $u$, i.e.,

$$
\Sigma_{0}=\left\{(x, t) \in \mathbf{T}^{n} \times[0, \infty] ; u^{*}(x, t)>u_{*}(x, t)\right\} .
$$

By Theorem 5.4 the solution $u$ has the regularity $\left(u_{*}\right)^{*}=u$. This implies that $\Sigma$ is a nowhere dense set. In other words $\Sigma$ has no interior. The convergence sg $u^{\varepsilon} \rightarrow \mathrm{sg}$ $u$ implies the local uniform convergence of $u^{\varepsilon}$ to $u$ outside $\Sigma$. (This yields Corollary 1.4.) We do not know whether or not the Lebesgue measure of $\Sigma$ is zero in our framework. Moreover, it is not known whether or not $u$ is a function of bounded variations when $u_{0}$ is a function of bounded variations. Also we do not know whether or not $u^{\varepsilon} \rightarrow u$ in $L^{1}$.

\subsection{Stability}

We construct a family of decreasing function $u$ satisfying

$$
h^{I}(u) \partial_{x} u=\varepsilon \partial_{x}^{2} u+h(u) \partial_{x} u, \quad x \in \mathbf{R}, u \in I=\left[a, z_{0}\right],
$$

where $\varepsilon$ is a positive small parameter. Here $h^{I}$ denote the derivative of $f_{I}$ and $f_{I}$ denotes the convex hull of $f$ in $I ; f$ is a primitive of $h$. If $h$ is decreasing so that $h^{I}$ is constant $-c$, then for a solution $u$ of $(6.3)$ the function $v(x, t)=u(x-c t)$ solves

$$
\partial_{t} v-h(v) \partial_{x} v=\varepsilon \partial_{x}^{2} v
$$

thus $u$ is regarded as a viscous shock travelling wave.

Instead of studying (6.3) we rather consider the equation for the inverse function $x=w(z)$ of $z=u(x)$. Differentiating $x=w(u(x))$ in $x$ to get

$$
\partial_{z} w \partial_{x} u=1 \text {. }
$$

Differentiating this identity again to get

$$
\partial_{z}^{2} w \cdot\left(\partial_{x} u\right)^{2}+\partial_{z} w \partial_{x}^{2} u=0 .
$$

From (6.4) and (6.5) it follows that

$$
\partial_{x} u=1 / \partial_{z} w \quad \text { and } \quad \partial_{x}^{2} u=-\partial_{z}^{2} w /\left(\partial_{z} w\right)^{3} .
$$


Plugging these identities into (6.3) we obtain

$$
\begin{aligned}
& h^{I}(z)=-\varepsilon \Delta w /\left(\partial_{z} w\right)^{2}+h(z) \quad \text { or } \\
& h^{I}(z)=\varepsilon \partial_{z}\left(1 / \partial_{z} w\right)+h(z)
\end{aligned}
$$

which is equivalent to (6.3). We shall study (6.6) instead of (6.3).

Lemma 6.6. Assume that $h$ is continuous in $I$. Let $I$ be a nontrivial interval $\left[a, z_{0}\right]$. For $\alpha>0$ let $w^{\varepsilon, \alpha}=w(z ; \varepsilon, \alpha)$ be a $\left(C^{2}\right)$ solution of $(6.6)$ in $I$ with $\partial_{z} w^{\varepsilon, \alpha}\left(z_{0}\right)=-\alpha$ and $w^{\varepsilon, \alpha}\left(z_{0}\right)=0$. Then

(i) $\partial_{z} w^{\varepsilon, \alpha}(z)<0$ for $z \in I, \varepsilon>0$.

(ii) $\partial_{z} w^{\varepsilon, \alpha}(z)=-\alpha$ if $f_{I}(z)=f(z)$, where $f$ is a primitive of $h$.

(iii) $-\alpha<\partial_{z} w^{\varepsilon, \alpha}(z) \uparrow 0$ as $\varepsilon \rightarrow 0$ if $f_{I}(z) \neq f(z)$.

(iv) $w^{\varepsilon, \alpha}$ converges uniformly in $I$ as $\varepsilon \rightarrow 0$ and the limit $w^{0, \alpha}$ is the Lipschitz function satisfying $\partial_{z} w^{0, \alpha}(z)=-\alpha$ if $f_{I}(z)=f(z)$ and $\partial_{z} w^{0, \alpha}(z)=0$ if $f_{I}(z) \neq f(z)$; moreover, $w^{0, \alpha}\left(z_{0}\right)=0$.

Proof. Integrate (6.6) from $z_{0}$ to $z$ to get

$$
\varepsilon\left(w^{\prime}\right)^{-1}=\varepsilon(-\alpha)^{-1}-f+f_{I}, f(z)=\int_{z_{0}}^{z} h(\tau) d \tau, w^{\prime}=\partial_{z} w
$$

or

$$
w^{\prime}(z)=\frac{-\alpha}{1+(-\alpha)\left(f_{I}-f\right) / \varepsilon} .
$$

Since $f_{I} \leq f$ by definition, (i)-(iii) follows directly from (6.7). Since

$$
\sup _{I}\left|w^{\varepsilon, \alpha}-w^{0, \alpha}\right| \leq \int_{I}\left|\partial_{z} w^{\varepsilon, \alpha}-\partial_{z} w^{0, \alpha}\right| d z
$$

the monotone convergence theorem with (ii), (iii) yields (iv).

Proof of Theorem 6.1. Since the proof for supersolutions is symmetric, we give the proof for subsolutions.

1. It is standard [4] that $\bar{u}$ is a subsolution. Indeed, it suffices to check (2.2) for $\varphi \in C^{2}(Q)$ and $(\hat{x}, \hat{t}) \in Q=\Omega \times(0, T)$ which is a strict maximizer of $\bar{u}-\varphi$ in $Q$. By the convergence of maximum points [4], there is a sequence $\left\{\left(x_{\varepsilon}, t_{\varepsilon}\right)\right\}$ which is a maximizer of $u^{\varepsilon}-\varphi$ and $\left(x_{\varepsilon}, t_{\varepsilon}\right) \rightarrow\left(x_{0}, t_{0}\right), u^{\varepsilon}\left(x_{\varepsilon}, t_{\varepsilon}\right) \rightarrow \bar{u}(\hat{x}, \hat{t})$. (We may assume that $u^{\varepsilon}$ is upper semicontinuous by taking its envelope.) Since $u^{\varepsilon}$ is a subsolution of $\partial_{t} u+H(u, \nabla u)=\varepsilon \Delta u, \varphi$ must satisfy

$$
\partial_{t} \varphi+H\left(u^{\varepsilon}, \nabla \varphi\right) \leq \varepsilon \Delta \varphi \quad \text { at } \quad\left(x_{\varepsilon}, t_{\varepsilon}\right)
$$


Letting $\varepsilon \rightarrow 0$ yields (2.2).

2. Let $\left\{S_{t}\right\}$ be an upper test surface of $\bar{u}$ at $P=(\hat{x}, \hat{t}) \in Q$ with level $\mu$. We may assume that $S_{t}$ is represented by $x_{1}=\psi\left(x^{\prime}, t\right), x^{\prime}=\left(x_{2}, \cdots, x_{n}\right)$ near $P$ and $\nabla_{x^{\prime}} \psi\left(\hat{x}^{\prime}, \hat{t}\right)=0$ so that $\mathbf{n}=\mathbf{n}(\hat{x}, \hat{t})=(1,0, \cdots, 0)$ by rotation of coordinates. We may assume that

$$
\operatorname{sg} \bar{u} \cap\left\{(x, t, z) ; x_{1} \geq \psi\left(x^{\prime}, t\right)\right\}=\{(P, z) ; \mu \leq z \leq \bar{u}(P)\}
$$

in $Z=I_{r}\left(\hat{x}_{1}\right) \times B_{r}\left(\hat{x}^{\prime}\right) \times I_{r}(\hat{t}) \times[\mu, \infty)$, where $I_{r}(q)=[q-r, q+r]$ for sufficiently small $r>0$. We define an upper semicontinuous function $\bar{u}^{\#}$ by

$$
\begin{aligned}
& \bar{u}^{\#}\left(x^{\prime}, t, z\right)=\sup \left\{x_{1} \in \mathbf{R} ;\left(x_{1}, x^{\prime}, t, z\right) \in Z \cap \operatorname{sg} \bar{u}, \hat{x}_{1}-r<x_{1} \leq \psi\left(x^{\prime}, t\right)\right\} \\
& \quad \text { for }\left(x^{\prime}, t, z\right) \in Z_{0}=B_{r}\left(\hat{x}^{\prime}\right) \times I_{r}(\hat{t}) \times[\mu, \infty) .
\end{aligned}
$$

We use the convention that $u^{\#}\left(x^{\prime}, t, z\right)=-\infty$ if the set $\{\cdot\}$ in the right hand side is empty. For fixed $\delta>0(\delta<r)$ we also define $u^{\varepsilon \#}$ by

$$
u^{\varepsilon \#}\left(x^{\prime}, t, z\right)=\sup \left\{x_{1} \in \mathbf{R} ;\left(x_{1}, x^{\prime}, t, z\right) \in Z \cap \operatorname{sg} u^{\varepsilon}, \hat{x}_{1}-r<x_{1} \leq \psi\left(x^{\prime}, t\right)+\delta\right\}
$$

for $\left(x^{\prime}, t, z\right) \in Z_{0}$. Since $\lim \sup ^{*} u^{\varepsilon}=\bar{u}$ is equivalent to $\lim \sup ^{*} i_{u^{\varepsilon}}=i_{\bar{u}}$, we see that $\lim \sup ^{*} u^{\varepsilon \#}=\bar{u}^{\#}$ as functions in $Z_{0}$.

3. For $I=I_{\eta}=[\mu, \bar{u}(P)+\eta]$ with $\eta>0$ and $\alpha>0$ let $w^{\varepsilon, \alpha}$ be the function defined in Lemma 6.6 with $h$ to be determined later. We fix $\alpha>0$ and for the moment we fix $\eta>0$. We set

$$
\varphi^{\varepsilon}\left(x^{\prime}, t, z\right)=w^{\varepsilon, \alpha}(z)+\psi\left(x^{\prime}, t\right), \quad\left(x^{\prime}, t, z\right) \in W=B_{r}\left(\hat{x}^{\prime}\right) \times I_{r}(\hat{t}) \times[\mu, \bar{u}(P)+\eta]
$$

and observe that $\varphi^{\varepsilon}$ converges to

$$
\varphi\left(x^{\prime}, t, z\right)=w^{0, \alpha}(z)+\psi\left(x^{\prime}, t\right)
$$

uniformly in $W$ as $\varepsilon \rightarrow 0$ by Lemma 6.6 (iv). By (6.8) $\bar{u}-\varphi$ takes its maximum over $W$ only at $x^{\prime}=\hat{x}^{\prime}, t=\hat{t}$ and for $z \in[\mu, \bar{u}(P)]$ with $w^{0, \alpha}(z)=0$. Assume that a primitive $f$ of $h$ satisfies the property that

$$
\left\{z \in Z ; f_{I}(z)=f(z)\right\} \quad \text { has the positive Lebesgue measure }
$$

so that $w^{0, \alpha}(z) \neq 0$ near $\mu$ by Lemma 6.6 (iv). Let $\left(x_{\varepsilon}^{\prime}, t_{\varepsilon}, z_{\varepsilon}\right)$ be a maximizer of $u^{\varepsilon \#}-\varphi^{\varepsilon}$ over $W$. Since $\lim \sup ^{*} u^{\varepsilon \#}=\bar{u}^{\#}$, by the convergence of maximum 
points [4], [17], $\left(x_{\varepsilon}^{\prime}, t_{\varepsilon}, z_{\varepsilon}\right)$ converges to a point $\left(\hat{x}^{\prime}, \hat{t}, z_{*}\right)$ with some $z_{*} \in(\mu, \bar{u}(P))$ satisfying $w^{0, \alpha}\left(z_{*}\right)=0$ by taking a subsequence if necessary. Moreover, $r_{\varepsilon}=\left(u^{\varepsilon \#}-\right.$ $\left.\varphi^{\varepsilon}\right)\left(x_{\varepsilon}^{\prime}, t_{\varepsilon}, z_{\varepsilon}\right) \rightarrow\left(\bar{u}^{\#}-\varphi\right)\left(\hat{x}^{\prime}, \hat{t}, z_{*}\right)(=0)$ as $\varepsilon \rightarrow 0$.

4. We turn to write these relations as functions of $\left(x_{1}, x^{\prime}, t\right)$. Let $q^{\varepsilon, \alpha}$ be the inverse function of $w^{\varepsilon, \alpha}$. We set

$$
\Phi^{\varepsilon}(x, t)=q^{\varepsilon, \alpha}\left(x_{1}-\psi\left(x^{\prime}, t\right)-r_{\varepsilon}\right)
$$

This is a smooth function. By the convergence of $\left(x_{\varepsilon}^{\prime}, t_{\varepsilon}, z_{\varepsilon}\right)$ we see that $u^{\varepsilon}-\Phi^{\varepsilon}$ takes its maximum 0 at $P_{\varepsilon}=\left(x_{1 \varepsilon}, x_{\varepsilon}^{\prime}, z_{\varepsilon}\right)$ with $x_{1 \varepsilon}=\psi\left(x_{\varepsilon}^{\prime}, t_{\varepsilon}\right)+r_{\varepsilon}+w^{\varepsilon, \alpha}\left(z_{\varepsilon}\right)$ over $I_{r}\left(\hat{x}_{1}\right) \times B_{r}\left(\hat{x}^{\prime}\right) \times I_{r}(\hat{t})$ for sufficiently small $\varepsilon>0$ and observe that $P_{\varepsilon} \rightarrow P$. Moreover, $u^{\varepsilon}\left(P_{\varepsilon}\right) \rightarrow z_{*} \in(\mu, \bar{u}(P))$ satisfying $w^{0, \alpha}\left(z_{*}\right)=0$. (If (6.9) is not fulfilled, $z_{*}$ may equal $\mu$ and $u^{\varepsilon}-\Phi^{\varepsilon}$ may not attain its maximum at an interior point of $I_{r}\left(\hat{x}_{1}\right) \times B_{r}\left(\hat{x}^{\prime}\right) \times I_{r}(\hat{t})$ even for small $\varepsilon>0$.)

5. Since $u^{\varepsilon}$ is a subsolution of $(6.1)$, we see

$$
\partial_{t} \Phi^{\varepsilon}\left(P_{\varepsilon}\right)+H\left(u^{\varepsilon}\left(P_{\varepsilon}\right), \nabla \Phi^{\varepsilon}\left(P_{\varepsilon}\right)\right)-\varepsilon \Delta \Phi^{\varepsilon}\left(P_{\varepsilon}\right) \leq 0
$$

By $q_{\varepsilon}, q_{\varepsilon}^{\prime}, q_{\varepsilon}^{\prime \prime}$ we mean $q^{\varepsilon, \alpha}, q^{\varepsilon, \alpha \prime}, q^{\varepsilon, \alpha \prime \prime}$ evaluated at $w^{\varepsilon, \alpha}\left(z_{\varepsilon}\right)=x_{1 \varepsilon}-\psi\left(x_{\varepsilon}^{\prime}, t_{\varepsilon}\right)-r_{\varepsilon}$. Dividing $-q_{\varepsilon}^{\prime}=-\left(q^{\varepsilon, \alpha}\right)^{\prime}\left(x_{1 \varepsilon}-\psi\left(x_{\varepsilon}^{\prime}, t_{\varepsilon}\right)+r_{\varepsilon}\right)$ to get

$$
\begin{aligned}
\partial_{t} \psi\left(x_{\varepsilon}^{\prime}, t_{\varepsilon}\right)+\left(-q_{\varepsilon}^{\prime}\right)^{-1} H\left(u^{\varepsilon}\left(P_{\varepsilon}\right), q_{\varepsilon}^{\prime}\left(1,-\nabla^{\prime} \psi\left(x_{\varepsilon}^{\prime}, t_{\varepsilon}\right)\right)\right. \\
-\varepsilon\left(-q_{\varepsilon}^{\prime}\right)^{-1} q_{\varepsilon}^{\prime \prime}\left(1+\left|\nabla^{\prime} \psi\left(x_{\varepsilon}^{\prime}, t_{\varepsilon}\right)\right|^{2}\right)-\varepsilon \Delta^{\prime} \psi\left(x_{\varepsilon}^{\prime}, t_{\varepsilon}\right) \leq 0
\end{aligned}
$$

where $\nabla^{\prime}$ and $\Delta^{\prime}$ denotes the gradient and the Laplacian in $x^{\prime}$. We shall send $\varepsilon$ to zero in (6.10). Since $P_{\varepsilon} \rightarrow P$, we see that $\partial_{t} \psi\left(x_{\varepsilon}^{\prime}, t_{\varepsilon}\right) \rightarrow \partial_{t} \psi\left(\hat{x}^{\prime}, \hat{t}\right)=V$, $\nabla^{\prime} \psi\left(x_{\varepsilon}^{\prime}, t_{\varepsilon}\right) \rightarrow \nabla^{\prime} \psi\left(\hat{x}^{\prime}, \hat{t}\right)=0, \Delta^{\prime} \psi\left(x_{\varepsilon}^{\prime}, t_{\varepsilon}\right) \rightarrow \Delta^{\prime} \psi\left(\hat{x}^{\prime}, \hat{t}\right)$. Since $u^{\varepsilon}\left(P_{\varepsilon}\right) \rightarrow z_{*}$ with $w^{0, \alpha}\left(z_{*}\right)=0$, the value $-q_{\varepsilon}^{\prime} \rightarrow+\infty$. By $(\mathrm{H} 3)$

$$
\begin{aligned}
& \left(-q_{\mathcal{\varepsilon}}^{\prime}\right)^{-1} H\left(u^{\varepsilon}\left(P_{\varepsilon}\right), q_{\varepsilon}^{\prime}\left(1,-\nabla^{\prime} \psi\left(x_{\varepsilon}^{\prime}, t_{\varepsilon}\right)\right)\right. \\
& \quad \rightarrow H_{\infty}\left(z_{*},(-1,0, \cdots, 0)\right) \text { as } \varepsilon \rightarrow 0
\end{aligned}
$$

Since $q^{\varepsilon, \alpha}$ solves (6.3), we see that

$$
\varepsilon\left(-q_{\varepsilon}^{\prime}\right)^{-1} q_{\varepsilon}^{\prime \prime}=h\left(q_{\varepsilon}\right)-h^{I}\left(q_{\varepsilon}\right)\left(=h\left(u^{\varepsilon}\left(P_{\varepsilon}\right)\right)-h^{I}\left(u^{\varepsilon}\left(P_{\varepsilon}\right)\right)\right)
$$

Combining these observations, we send $\varepsilon$ to zero in (6.10) to get

$$
V+H_{\infty}\left(z_{*},-\mathbf{n}\right)-h\left(z_{*}\right)+h^{I}\left(z_{*}\right) \leq 0 .
$$


If $z \mapsto H_{\infty}(z,-\mathbf{n})$ satisfies the property (6.9) (for its primitive) with $I=I_{0}=$ $[\mu, \bar{u}(P)]$, we see that it also satisfies (6.9) with $I=I_{\eta}=[\mu, \bar{u}(P)+\eta]$. We may take $h(z)=H_{\infty}(z,-\mathbf{n})$ to get

$$
V+H^{I}\left(z_{*},-\mathbf{n}\right) \leq 0, I=I_{\eta}
$$

where $H^{I}$ denotes the relaxed Hamiltonian. Since $w^{0, \alpha}=0$ on $\left[z_{*}, \bar{u}(P)\right], h^{I}$ is constant in $\left.\left[z_{*}, \bar{u}(P)\right)\right]$ by Lemma 6.6 (iv). We now obtain

$$
V+H^{I}(\bar{u}(P),-\mathbf{n}) \leq 0 \quad \text { with } I=I_{\eta}=[\mu, \bar{u}(P)+\eta]
$$

We send $\eta \rightarrow 0$ to get

$$
V+H^{I_{0}}(\bar{u}(P),-\mathbf{n}) \leq 0, I_{0}=[\mu, \bar{u}(P)]
$$

by continuity of $H^{I}$ in $I$ (Lemma 2.1 (iv)). This inequality is exactly what we would like to derive.

6. It remains to handle the case that $\tilde{h}(z)=H_{\infty}(z,-\mathbf{n})$ does not satisfy (6.9), i.e. the set $\left\{z \in I_{0} ; f_{I_{0}}(z)=f(z)\right\}$ has the zero Lebesgue measure for a primitive $f$ of $\tilde{h}$. Modifying $\tilde{h}$ near $\mu$ properly, it is not difficult to find a family $\left\{h_{\delta}\right\}_{\delta>0} \subset C(I)$ such that

$$
h_{\delta}(z) \leq \tilde{h}(z) \quad \text { in } \quad I_{\eta}, h_{\delta}(z)=\tilde{h}(z) \text { for } z \geq \mu+\delta,
$$

and moreover $h_{\delta}$ satisfies (6.9) for $I=I_{0}$. We take $h=h_{\delta}$ in (6.11) to obtain

$$
V+h_{\delta}^{I}\left(z_{*}\right) \leq 0, I=I_{\eta}
$$

since $H_{\infty}\left(z_{*},-\mathbf{n}\right) \geq h_{\delta}\left(z_{*}\right)$ by the choice of $h_{\delta}$. Since $h_{\delta}^{I}$ is constant in $\left[z_{*}, \bar{u}(P)\right]$ for small $\delta>0$ this implies

$$
V+h_{\delta}^{I}(\bar{u}(P)) \leq 0 .
$$

It is possible to take $h_{\delta}$ such that $h_{\delta}^{I}(\bar{u}(P)) \rightarrow \tilde{h}^{I}(\bar{u}(P))$ as $\delta \rightarrow 0$. Thus we obtain (6.12). As in Step 5 sending $\eta \rightarrow 0$ yields (6.13).

Proof of Theorem 6.2. We only present the proof for a subsolution since the proof for a supersolution is symmetric. In the case of $\mathbf{T}^{n}$ we interpret $u_{0}$ as a periodic function in $\mathbf{R}^{n}$, i.e.

$$
u_{0}(x)=u_{0}\left(x+\omega_{i} e_{i}\right)
$$

Let $\xi$ be a point in $\mathbf{R}^{n}$. By definition for each $\eta>0$ there is $\varepsilon_{1} \in\left(0, \varepsilon_{0}\right)$ such that

$$
u_{0}^{\varepsilon}(x)-u_{0}(\xi) \leq \eta
$$


if $|x-\xi|<\varepsilon_{1}$ and $\varepsilon<\varepsilon_{1}$. In other words for each $\delta \in(0,1)$ and a function $g \in C^{\infty}(\mathbf{R})$ with $g(0)=0, g^{\prime}>0$ there is a constant $A_{\delta \xi}>0$ that satisfies

$$
u_{0}^{\varepsilon}(x) \leq u_{0}(\xi)+\delta+A_{\delta \xi} g\left(|x-\xi|^{2}\right)
$$

for all $x \in \mathbf{R}^{n}$ and $\varepsilon<\varepsilon_{0}$. Since $u_{0}^{\varepsilon}$ is uniformly bounded on $\mathbf{R}^{n}$ for $\varepsilon<\varepsilon_{0}$, we may take that $g^{\prime}(s)$ is constant for sufficiently large $s>0$. By continuity of $H$ there is a large number $B_{0}=B_{0}\left(A_{\delta \xi}, g, R, T\right)$ independent of $\varepsilon<\varepsilon_{0}$ such that the function

$$
v_{\delta}(x, t)=B t+u_{0}(\xi)+\delta+A_{\delta \xi} g\left(|x-\xi|^{2}\right) \text { with } B>B_{0}
$$

fulfills

$$
\partial_{t} v_{\delta}+H\left(u^{\epsilon}, \nabla v_{\delta}\right)-\varepsilon \Delta v_{\delta}>0 \text { in } B_{R}(\xi) \times(0, T]
$$

for $\varepsilon<\varepsilon_{0}$ since $\left\{u^{\varepsilon}\right\}$ is uniformly bounded. Suppose that $\sup _{\mathbf{R}^{n} \times[0, T]}\left(u^{\varepsilon}-v_{\delta}\right)>0$ for some $\varepsilon>0$. Since $\left\{u^{\varepsilon}\right\}$ is uniformly bounded from above, $u^{\varepsilon}<v_{\delta}$ outside $\operatorname{int} B_{R}(\xi) \times[0, T]$ for some $R>0$. Since $\left(u^{\varepsilon}-v_{\delta}\right)^{*} \leq 0$ at $t=0$, there is a maximizer of $u^{\varepsilon}-v_{\delta}$ over $\operatorname{int} B_{R}(\xi) \times(0, T]$. Since $u^{\varepsilon}$ is a subsolution of $(6.1)$ and $v_{\delta}$ is smooth, it contradicts $(*)$. Thus we see that

$$
u^{\varepsilon}(x, t) \leq v_{\delta}(x, t), x \in \mathbf{R}^{n}, t>0 .
$$

Taking limsup* we obtain

$$
\bar{u}(\xi, 0) \leq\left(\limsup _{\varepsilon \rightarrow 0}^{*} v_{\delta}\right)(\xi, 0)=u_{0}(\xi)+\delta .
$$

Since $\delta>0$ is arbitrary, we conclude $\bar{u}(\xi, 0) \leq u_{0}(\xi)$ for $\xi \in \mathbf{R}^{n}$.

\subsection{Convergence}

We shall prove Theorem 6.4 (i). For this purpose we note a relation of limsup*, $\liminf _{*}$ and Hausdorff convergence of subgraphs.

Lemma 6.7. Let $K$ be a compact metric space. Assume that $\left\{w^{\varepsilon}\right\}_{0 \leq \varepsilon \leq 1}$ is a bounded family of upper semicontinuous functions on $K$. Then $\mathrm{sg} w^{\varepsilon} \rightarrow \mathrm{sg}$ $w^{0}(\varepsilon \rightarrow 0)$ in the Hausdorff distance sense in $K \times \mathbf{R}$ if and only if $w^{0}=\lim \sup ^{*} w^{\varepsilon}$ and $w^{0}=\left(\liminf { }_{*} w^{\varepsilon}\right)^{*}$. Similarly, the condition $\left(\limsup { }^{*} w^{\varepsilon}\right)_{*}=\liminf _{*} w^{\varepsilon}\left(=v^{0}\right)$ is equivalent to $S g w_{*}^{\varepsilon} \rightarrow S g v^{0}$.

Proof. We shall prove the 'if' part. Let $\chi_{\varepsilon}$ be the characteristic function of sg $w^{\varepsilon}$, i.e., $\chi_{\varepsilon}(z, y)=1$ if $y \leq w^{\varepsilon}(z)$ and otherwise $\chi_{\varepsilon}(z, y)=0$. Note that 
$w^{0}=\limsup ^{*} w^{\varepsilon}$ is equivalent to $\lim \sup ^{*} \chi_{\varepsilon}=\chi_{0}$ and that $w^{0}=\left(\liminf _{*} w^{\varepsilon}\right)^{*}$ is equivalent to $\left(\liminf _{*} \chi_{\varepsilon}\right)^{*}=\chi_{0}$. Since limsup* $\chi_{\varepsilon}=\chi_{0}$ implies that for $\eta>0$, there is $\varepsilon(\eta)>0$ such that

$$
\sup _{z \in \operatorname{sg} w^{\varepsilon}} \operatorname{dist}\left(z, \operatorname{sg} w^{0}\right) \leq \eta \quad \text { for } \varepsilon<\varepsilon(\eta)
$$

it suffices to prove that for any point $z_{0} \in \operatorname{sg} w^{0}$ and $\eta>0$ there is $\varepsilon^{\prime}(\eta)$ such that $B_{\eta}\left(z_{0}\right) \cap \mathrm{sg} w^{\varepsilon} \neq \emptyset$ for $\varepsilon<\varepsilon^{\prime}(\eta)$. If not, there is a ball $B_{\eta}\left(z_{0}\right)$ for some point $z_{0} \in \mathrm{sg}$ $w^{0}$ such that $B_{\eta}\left(z_{0}\right) \cap \mathrm{sg} w^{\varepsilon_{j}}=\emptyset$ for some subsequence $\varepsilon_{j} \rightarrow 0$. This implies that $\liminf _{*} \chi_{\varepsilon}=0$ on $B_{\eta}\left(z_{0}\right)$ which contradicts $\left(\liminf _{*} \chi_{\varepsilon}\right)^{*}=\chi_{0}$. We thus observe that $\operatorname{sg} w^{\varepsilon} \rightarrow \operatorname{sg} w^{0}$.

The 'only if' part is proved by deriving limsup* $\chi_{\varepsilon}=\chi_{0}$ and $\left(\liminf { }_{*} \chi_{\varepsilon}\right)^{*}=\chi_{0}$ from $\mathrm{sg} w^{\varepsilon} \rightarrow \operatorname{sg} w^{0}$ by tracing above arguments in the reverse way. The proof for supergraphs is symmetric so it is safely left to the reader.

Proof of Theorem 6.4 (i). Since $\left\{u^{\varepsilon}\right\}_{\varepsilon>0}$ is a bounded family of functions on $K=\mathbf{T}^{n} \times\left[0, T^{\prime}\right]$ for each $T^{\prime} \in(0, T)$. Thus $\bar{u}=\limsup _{\varepsilon \rightarrow 0}^{*} u^{\varepsilon}$ and $\underline{u}=\liminf _{* \varepsilon \rightarrow 0} u^{\varepsilon}$ are bounded on $K$. By stability (Theorem 6.1) $\bar{u}$ (resp. $\underline{u}$ ) is a proper subsolution (resp. supersolution) of $(4.1)$ in $\mathbf{T}^{n} \times(0, \infty)$. By Theorem 6.2 we see that

$$
\bar{u}(x, 0) \leq u_{0}(x), \underline{u}(x, 0) \geq u_{0 *}(x), x \in \mathbf{T}^{n} .
$$

Since

$$
\left.(\underline{u})^{*}\right|_{t=0} \geq\left(\left.\underline{u}\right|_{t=0}\right)^{*} \geq\left(u_{0 *}\right)^{*}=u_{0} \geq\left.\bar{u}\right|_{t=0},
$$

we now apply the proper comparison principle (Theorem 5.1) to get $\bar{u} \leq(\underline{u})^{*}$ on $\mathbf{T}^{n} \times$ $[0, T)$. Since $\bar{u} \geq(\underline{u})^{*}$ by definition, we obtain $\bar{u}=(\underline{u})^{*}$. The Hausdorff convergence result follows from Lemma 6.7 . Similarly, by using the proper comparison principle (Theorem 5.1) we obtain $\underline{u}=(\bar{u})_{*}$. Thus $\bar{u}$ is the upper semicontinuous proper solution of (4.1) with initial data $u_{0}$. The uniqueness follows from Theorem 5.4.

The proof for homogeneous equations is the same except we use Theorem 5.9 instead of Theorem 5.1. For the uniqueness see Remark 5.10.

Remark 6.8. Since $\underline{u}=(\bar{u})_{*}$ from Lemma 6.7 it also follows that $\mathrm{Sg} u^{\varepsilon} \rightarrow \mathrm{Sg}$ $u_{*}$. Thus graph $u^{\varepsilon} \rightarrow \operatorname{Sg} u_{*} \cap \operatorname{sg} u$ in the sense of Hausdorff distance topology in $\mathbf{T}^{n} \times[0, T] \times \mathbf{R}$ as $\varepsilon \rightarrow 0$, where

$$
\operatorname{graph} u^{\varepsilon}=\left\{(x, t, y) ; y=u^{\varepsilon}(x, t), x \in \mathbf{T}^{n}, t \geq 0\right\}
$$


Remark 6.9 (Generalization). (i) From the proof it is easy to see that our stability result (Theorem 6.1) is still valid for general equation (2.1) with continuous $H$ satisfying (2.8).

(ii) Even if one replaces the Laplacian in the diffusion term of (6.1) by more general uniformly elliptic operator with smooth coefficient, the proof of our stability result (Theorem 6.1) still applies with minor modifications to get the stability and consequently the convergence follows. It is interesting to consider nonlinear diffusion term. We do not pursue this problem here and shall study in one of our forthcoming papers.

\subsection{Monotone initial data}

We shall state our convergence and existence results for homogeneous equations when the initial data is strictly spokewise decreasing or increasing. Since the results for increasing data is symmetric, we only state them for decreasing data.

Theorem 6.10. Assume(H1) and (H2). Assume that (4.1) is homogeneous. Let $u_{0}$ be a bounded upper semicontinuous function on $\mathbf{R}^{n}$ with $\left(u_{0 *}\right)^{*}=u_{0}$. Assume that $u_{0}$ is strictly spokewise decreasing. Assume that $u_{0}$ is locally constant and continuous outside some ball $B_{R_{0}}(0)$.

(i) (Convergence). For bounded bounded function $u_{0}^{\varepsilon}(\varepsilon \in(0,1))$ let $u^{\varepsilon} \in C\left(\mathbf{R}^{n} \times\right.$ $[0, \infty))$ be a viscosity solution of $(6.1)$ with $\left.u^{\varepsilon}\right|_{t=0}=u_{0}^{\varepsilon}$. Assume that $u^{\varepsilon}$ is uniformly continuous on $\mathbf{R}^{n} \times[0, T]$ for each $T>0$ and for small $\varepsilon>0$. Assume that $u_{0}=\limsup _{\varepsilon \rightarrow 0}^{*} u_{0}^{\varepsilon}$ and $\left(u_{0}\right)_{*}=\liminf _{* \varepsilon \rightarrow 0} u_{0}^{\varepsilon}$. Assume that the convergence $u_{0}^{\varepsilon} \rightarrow u_{0}$ is uniform outside $B_{2 R_{0}}(0)$. Then sg $u^{\varepsilon}$ converges to $\mathrm{sg} u$ in the sense of Hausdorff distance topology in $B_{\rho}(0) \times \mathbf{R} \times[0, T]$ for any $T>0$ and $\rho>0$ as $\varepsilon \rightarrow 0$ with some upper semicontinuous function $u$ (independent of $\rho$ ). The limit $u$ is a unique bounded (upper semicontinuous) proper solution of (4.1) with initial data $u_{0}$ (in the sense of Definition 5.13). Moreover, $u(\cdot, t)$ is strictly spokewise decreasing in $\mathbf{R}^{n}$ for all $t \geq 0$. Furthermore, for any $T>0$ the function $u$ is locally constant and continuous on $\left(\mathbf{R}^{n} \backslash B_{R}(0)\right) \times[0, T]$ for some $R>0$.

(ii) (Existence). If $H(r, p)$ is Lipschitz in $p \in \mathbf{R}^{n}$ uniformly in $r \in(-R, R)$ for each $R>0$, then there exists a unique bounded proper (upper semicontinuous) solution $u$ of (4.1) with initial data $u_{0}$. The function $u(\cdot, t)$ is strictly spokewise decreasing in $\mathbf{R}^{n}$ for all $t \geq 0$.

Since $u_{0} \in C\left(\mathbf{R}^{n}\right)$ implies $\left(u_{0 *}\right)^{*}=u_{0}$, Theorem 6.10 yields Theorems 1.2 and 1.3 for (1.6) for strictly spokewise decreasing initial data. As pointed out in Remark 
6.8 , we also have the convergence of supergraphs and also the convergence graph $u^{\varepsilon} \rightarrow \operatorname{Sg} u_{*} \cap \operatorname{sg} u$.

Proof. (i) By Lemma 6.7 to show the convergence it suffices to prove that $\bar{u}=(\underline{u})^{*}$ for $\bar{u}=\limsup _{\varepsilon \rightarrow 0}^{*} u^{\varepsilon}$ and $\underline{u}=\liminf _{* \varepsilon \rightarrow 0} u^{\varepsilon}$. Let $M=\max u_{0}$ and $m=\min u_{0 *}$. By comparing with constant solutions $M$ and $m$, we see that $m \leq u^{\varepsilon} \leq M$ on $\mathbf{R}^{n} \times[0, \infty)$ so that $m \leq \underline{u} \leq \bar{u} \leq M$ there. (Indeed, if $u^{\varepsilon} \leq M$ does not hold, we consider a maximizer $(\hat{x}, \hat{t})$ of $u^{\varepsilon}(x, t)-M-\sigma t-\delta|x|^{2}$ in $\mathbf{R}^{n} \times[0, T]$ for $\delta>0$ and small $\sigma>0$ so that $\hat{t}>0$. Since $u^{\varepsilon}$ is a subsolution of (6.1), we have $\sigma+H\left(u^{\varepsilon}(\hat{x}, \hat{t}), 2 \delta \hat{x}\right) \leq 2 n \delta$. Letting $\delta \rightarrow 0$ yields a contradiction $\sigma \leq 0$. The proof for $m \leq u^{\varepsilon}$ is symmetric.) As in the proof of Theorem 6.4 (i) we are able to prove $\bar{u} \leq(\underline{u})^{*}$ by using the proper comparison principle (Theorem 5.11 and Remark 5.12) provided that for each $T>0$ functions $\bar{u}$ and $\underline{u}$ are locally constant and continuous on $\left(\mathbf{R}^{n} \backslash B_{R}(0)\right) \times[0, T]$ for some $R>0$. Since $\bar{u} \geq(\underline{u})^{*}$ this yields $\bar{u}=(\underline{u})^{*}$. The property that $u=\bar{u}(\cdot, t)$ is strictly spokewise decreasing in $\mathbf{R}^{n}$ for $t \geq 0$ is proved by comparing $\bar{u}$ and $\underline{u}_{\lambda}$ for $\lambda \in(0,1)$ by the weak comparison principle (Theorem 5.7), where $\underline{u}_{\lambda}(x, t)=\underline{u}(\lambda x, \lambda t)$. The uniqueness of a proper solution follows from Theorem 5.14.

It remains to prove the constancy of $\bar{u}$ and $\underline{u}$ near spatial infinity. We observe that if

$$
u_{0}(x)=m \text { for } x \cdot e \geq R_{1}
$$

then for each $T>0$ there exists a constant $R=R\left(R_{1}, M, T, H\right)$ (independent of the unit vector $e \in \mathbf{R}^{n}$ ) that satisfies

$$
\bar{u}(x, t)=m \text { for } x \cdot e \geq R, \quad t \in[0, T]
$$

provided that the convergence $u_{0}^{\varepsilon} \rightarrow u_{0}$ is uniform for $x$ satisfying $x \cdot e \geq R_{1}$. This argument does not require spokewise monotonicity of $u_{0}$. Indeed, $u^{\varepsilon}$ is a viscosity subsolution of

$$
\partial_{t} u-\alpha|\nabla u|=\varepsilon \Delta u \quad \text { in } \mathbf{R}^{n} \times(0, T)
$$

with

$$
\alpha=\inf \left\{H(r, p) /|p| ; m \leq r \leq M, p \in \mathbf{R}^{n}, p \neq 0\right\} .
$$

Let $v^{\varepsilon}=v^{\varepsilon}(\eta, t)$ be the solution of the heat equation

$$
\partial_{t} v-\varepsilon \partial_{\eta}^{2} v=0
$$


with initial condition

$$
v^{\varepsilon}(\eta, 0)= \begin{cases}m+\delta, & \eta>0 \\ M, & \eta<0\end{cases}
$$

for $\delta>0$. We set

$$
w^{\varepsilon}(x, t)=v^{\varepsilon}(x \cdot e-\alpha t, t)
$$

and observe that $w^{\varepsilon}$ is a solution of (6.16). By comparison principle for (6.16) (e.g. [10]) we see that

$$
u^{\epsilon} \leq w^{\epsilon} \quad \text { on } \mathbf{R}^{n} \times[0, T)
$$

for sufficiently small $\varepsilon>0$. Since $v^{\varepsilon} \rightarrow m+\delta$ locally uniform on $\left\{x_{1}>0\right\}$, this implies $\bar{u} \leq m+\delta$ for $x$ satisfying $x \cdot e \geq R:=R_{1}-\alpha T$. Since $\delta>0$ is arbitrary, we now obtain (6.15).

The implication of (6.14) to (6.15) is still valid by replacing $m$ by $M$ and $\bar{u}$ by $\underline{u}$. These properties evidently yield the constancy of $\bar{u}$ and $\underline{u}$ near spatial infinity. (ii) As in Proposition 6.3 a standard argument (see e.g. [36, Proposition 7.3.6]) yields the unique existence of $C^{2,1}$ solution $u^{\varepsilon}$ of (6.1) which is bounded uniformly continuous on $\mathbf{R}^{n} \times[0, T]$ for any $T>0$ when the initial data $u_{0}$ is bounded uniformly continuous. Thus, (ii) follows from (i).

From the proof of Theorem 6.4 (i) and Theorem 6.10 (i) we see that the convergence follows from stability (Theorem 6.1), nonexistence of initial layers (Theorem 6.2) and the proper comparison principle. Although Theorems 6.1 and 6.2 do not need many assumptions of equations and initial data, we are forced to restrict the situation for convergence since the proper comparison principle has been proved only for restrictive situations. We conclude this section to give a convergence result admitting the proper comparison principle.

Theorem 6.11. Assume that (H1) and (H3). Let $u_{0}$ be an upper semicontinuous function on $\mathbf{R}^{n}$ satisfying $\left(u_{0 *}\right)^{*}=u_{0}$. Assume that for any $v_{-}$and $v_{+}$satisfying $\left.\left(v_{-}^{*}\right)\right|_{t=0} \leq u_{0}$ and $\left.\left(v_{+*}\right)\right|_{t=0} \geq u_{0 *}$ the inequality

$$
v_{-}^{*} \leq\left(v_{+*}\right)^{*} \quad \text { in } \mathbf{R}^{n} \times(0, T)
$$

holds provided that $v_{-}$and $v_{+}$are proper sub- and supersolutions of (4.1) in $\mathbf{R}^{n} \times$ $(0, T)$, respectively. Let $u^{\varepsilon} \in C\left(\mathbf{R}^{n} \times[0, T)\right)$ be a viscosity solution of $(6.1)$ in $\mathbf{R}^{n} \times(0, T)$ with $u_{0}^{\varepsilon}=\left.u^{\varepsilon}\right|_{t=0} \in C\left(\mathbf{R}^{n}\right)$. Assume that $u_{0}=\limsup ^{*} u_{0}^{\varepsilon}$. Assume that $\left\{u^{\varepsilon}\right\}$ is uniformly bounded on $\mathbf{R}^{n} \times[0, T)$ for small $\varepsilon>0$. Then $\bar{u}=(\underline{u})^{*}$ in $\mathbf{R}^{n} \times[0, T)$ for $\bar{u}=\limsup ^{*} u^{\varepsilon}, \underline{u}=\liminf _{*} u^{\varepsilon}$. 


\section{Scalar conservation law}

A scalar conservation law

$$
\partial_{t} u+\sum_{i=1}^{n} \partial_{x_{i}}\left(f^{i}(u)\right)=0
$$

is a typical example of (4.1) with

$$
H(r, p)=\sum_{i=1}^{n}\left(\partial_{r} f^{i}\right)(r) p_{i}
$$

The equation is homogeneous and it satisfies (H1) and (H2) if $f^{i}$ 's is $C^{2}$. However, it does not fulfill ( $\mathrm{H} 5 \mathrm{a})$ or $(\mathrm{H} 5 \mathrm{~b})$ unless $f^{i}$ 's is a constant. Thus Theorem 6.4 does not apply. Fortunately, Theorem 6.10 (or Theorem 1.2) applies to (7.1), (7.2). Thus, except the last statement Corollary 1.5 has been proved. For (7.1) there is a notion of entropy (or admissible) solutions which characterizes the limit of a solution of (6.1) with (7.2) i.e.

$$
\partial_{t} u+\sum_{i=1}^{n} \partial_{x_{i}}\left(f^{i}(u)\right)=\varepsilon \Delta u
$$

as $\varepsilon \rightarrow 0$. The global unique existence of the entropy solution has been established as well as various properties of solutions. We refer a recent book of Dafermos [12] for the state of arts. We shall prove that our limit $u$ in Corollary 1.5 is an entropy solution. It looks trivial; however, it is not trivial since the convergence sg $u^{\varepsilon} \rightarrow \mathrm{sg}$ $u$ may not imply $L^{1}$-convergence or almost everywhere convergence.

We first recall that the entropy solution is obtained by the vanishing viscosity method which was first proved by Kružkov [33].

Proposition 7.1. Assume that $f^{i} \in C^{2}(\mathbf{R}), i=1,2, \cdots, n$. For $u_{0} \in L^{\infty}\left(\mathbf{R}^{n}\right)$ let $u^{\varepsilon}$ be the solution of (7.3) which initially equals to $u_{0}$. Then $u^{\varepsilon}$ subsequently converges to a function $u$ as $\varepsilon \rightarrow 0$ almost everywhere. Moreover,

$$
\int_{0}^{T} \int_{B_{R}(0)}\left|u^{\varepsilon}-u\right| d x d t \rightarrow 0 \quad \text { as } \varepsilon \rightarrow 0
$$

for every $R>0, T>0$.

Note that the existence of the solution $u^{\varepsilon}$ of (7.3) follows from a variant of Proposition 6.3 if $f^{i}$ 's is $C^{2}$. 
The function $u$ is called the entropy solution of (7.1) with initial data $u_{0}$. It is a special weak solution and characterized without limiting procedure [33], [12]. The entropy solution has an $L^{1}$ contraction property which yields its uniqueness.

Proposition 7.2. Assume that $f^{i} \in C^{1}(\mathbf{R}), i=1, \cdots, n$. For $u_{0}, \tilde{u}_{0} \in L^{\infty}\left(\mathbf{R}^{n}\right)$ let $u$ and $\tilde{u}$ be an entropy solution with initial data $u_{0}$ and $\tilde{u}_{0}$ for (7.1), respectively. Then

$$
\int_{\mathbf{R}^{n}}|u(x, t)-\tilde{u}(x, t)| d x \leq \int_{\mathbf{R}^{n}}\left|u_{0}(x)-\tilde{u}_{0}(x)\right| d x .
$$

Moreover, $t \mapsto u(\cdot, t)$ is continuous in $[0, \infty)$ with respect to $L^{1}\left(\mathbf{R}^{n}\right)$-norm.

Based on these results we are able to prove that the entropy solution agrees with our proper viscosity solutions almost everywhere (a.e.) for suitable initial data.

Theorem 7.3. Assume that $f^{i} \in C^{2}(\mathbf{R})$ for $i=1, \cdots, n$. Let $u_{0}$ be a bounded upper semicontinuous function on $\mathbf{R}^{n}$ such that $\left(u_{0 *}\right)^{*}=u_{0}$. Assume that $u_{0}$ is locally constant and continuous outside some ball.

(i) Let $u^{\varepsilon}$ be the classical solution of (7.3) and the initial condition $u^{\varepsilon *}(x, 0)=u_{0}(x)$. Let $\bar{u}=\limsup _{\varepsilon \rightarrow 0}^{*} u^{\varepsilon}$. Let $u$ be the entropy solution of (7.1) with initial data $u_{0}$. Then $u=\bar{u}$ a.e. in $\mathbf{R}^{n} \times(0, \infty)$. Moreover, for $T>0, \bar{u}$ and $\underline{u}$ are locally constant and continuous on $\left(\mathbf{R}^{n} \backslash B_{R}(0)\right) \times[0, T]$ for some $R>0$.

(ii) Assume moreover that $u_{0}=u_{0 *}$ a.e. in $\mathbf{R}^{n}$. Then $u=\underline{u}=\bar{u}$ a.e. in $\mathbf{R}^{n} \times(0, \infty)$, where $\underline{u}=\liminf _{\varepsilon \rightarrow 0} u^{\varepsilon}$. In particular $u$ is continuous except some set $S$ of Lebesgue measure zero.

(iii) Under assumptions of (i), (ii) the identities $(\bar{u})_{*}=\left((\underline{u})^{*}\right)_{*},(\underline{u})^{*}=\left((\bar{u})_{*}\right)^{*}$ holds everywhere in $\mathbf{R}^{n} \times[0, \infty)$.

Proof. (i) Let $R_{0}>0$ be a number such that $u_{0}$ is locally constant outside $B_{R_{0}}(0)$. For $\delta>0$ let $u_{0 \delta}$ denote the upper semicontinuous function defined by

$$
u_{0 \delta}(x)= \begin{cases}\sup \left\{y ; \operatorname{dist}\left((x, y), \operatorname{sg} u_{0}\right) \leq \delta\right\}, & x \in B_{2 R_{0}}(0), \\ u_{0}(x), & x \in \mathbf{R}^{n} \backslash B_{2 R_{0}}(0) .\end{cases}
$$

By definition $\left(u_{0 \delta *}\right)^{*}=u_{0 \delta}$. Let $u_{\delta}^{\varepsilon}$ denote the solution of (7.3) starting with $u_{0 \delta}$. Since $u^{\varepsilon}$ and $u_{\delta}^{\varepsilon}$ subsequently converge to entropy solutions $u$ and $u_{\delta}$ of (7.1) with initial data $u_{0}$ and $u_{0 \delta}$, respectively, as $\varepsilon \rightarrow 0$ almost everywhere in $\mathbf{R}^{n} \times(0, \infty)$ by Proposition 7.1, we see that

$$
u \leq \bar{u} \text { and } \underline{u}_{\delta} \leq u_{\delta} \text { a.e. in } \mathbf{R}^{n} \times(0, \infty)
$$


where $\underline{u}_{\delta}=\liminf _{\varepsilon \rightarrow 0} u_{\delta}^{\varepsilon}$. Note that $\underline{u}_{\delta}$ is a proper supersolution and $\bar{u}$ is a proper subsolution by Theorem 6.1. Since (6.14) yields (6.15), the functions $\bar{u}$ and $\underline{u}$ are locally constant and continuous on $\left(\mathbf{R}^{n} \backslash B_{R}(0)\right) \times[0, T]$ for some $R>0$. Since $\left.\underline{u}_{\delta}\right|_{t=0} \geq\left(u_{0 \delta}\right)_{*}$ by Theorem 6.2 and $u_{0 \delta *}>u_{0}^{*}$, by the weak comparison principle (Theorem 5.7) we have $\bar{u}<\underline{u}_{\delta}$ in $\mathbf{R}^{n} \times(0, \infty)$. We thus obtain

$$
u \leq \bar{u} \leq u_{\delta} \quad \text { a.e. in } \mathbf{R}^{n} \times(0, \infty)
$$

By definition $i_{u_{0 \delta}}-i_{u_{0}^{*}} \rightarrow 0$ at all points, so

$$
\int_{\mathbf{R}^{n}}\left|u_{0 \delta}-u_{0}\right| d x \rightarrow 0 \text { as } \delta \rightarrow 0
$$

By the contraction principle (Proposition 7.2)

$$
\sup _{0 \leq t \leq T} \int_{\mathbf{R}^{n}}\left|u_{\delta}(x, t)-u(x, t)\right| d x \leq \int_{\mathbf{R}^{n}}\left|u_{0 \delta}-u_{0}\right| d x \rightarrow 0 .
$$

Thus we conclude that $u=\bar{u}$ a.e. in $\mathbf{R}^{n} \times(0, \infty)$.

(ii) Let $v$ be the entropy solution of (7.1) with initial data $u_{0 *}$. A symmetric argument to (i) implies that $v=\underline{u}$ a.e. in $\mathbf{R}^{n} \times(0, \infty)$. Since $u_{0 *}=u_{0}^{*}$ a.e. in $\mathbf{R}^{n}, v=u$ a.e. in $\mathbf{R}^{n} \times(0, \infty)$ by the uniqueness of the entropy solution (Proposition 7.2). We thus observe that $\bar{u}=\underline{u}=u$ a.e. in $\mathbf{R}^{n} \times(0, \infty)$. The remaining statement easily follows from $\bar{u}=\underline{u}=u$ a.e.

(iii) If $(\bar{u})_{*}>(\underline{u})^{*}$ at some point $(\hat{x}, \hat{t}) \in \mathbf{R}^{n} \times(0, T)$, then $\bar{u}>\underline{u}$ near $(\hat{x}, \hat{t})$. This contradicts $\bar{u}=\underline{u}$ a.e. so we have $(\bar{u})_{*} \leq(\underline{u})^{*}$. Since $\underline{u} \leq \bar{u}$ implies $\left((\underline{u})^{*}\right)_{*} \leq(\bar{u})_{*}$, the identity $(\underline{u})^{*}=\left((\bar{u})_{*}\right)^{*}$ follows from $(\bar{u})_{*} \leq(\underline{u})^{*}$ and $(\underline{u})^{*} \leq\left((\bar{u})_{*}\right)^{*}$. The proof for $(\bar{u})_{*}=\left((\underline{u})^{*}\right)_{*}$ is symmetric.

Remark 7.4. (i) (Existence) It turns out that $U=(\underline{u})^{*}$ is an upper semicontinuous proper solution of (7.1) with initial data $u_{0}$ (in the sense of Definition 5.13) and it satisfies $\left(U_{*}\right)^{*}=U$. This yields the existence of a proper solution under the assumptions $\left(u_{0 *}\right)^{*}=u_{0}$ everywhere and $u_{0 *}=u_{0}$ a.e. without assuming monotonicity of $u_{0}$. (Since $\underline{u} \leq(\underline{u})^{*} \leq \bar{u}$ everwhere, by Theorem 7.3 (ii) $U$ agrees with the entropy solution with initial data $u_{0}$ almost everywhere.)

We shall give a sketch of the proof. We approximate $u_{0}$ from below by

$$
u_{0}^{\sigma}(x)= \begin{cases}\inf \left\{y ; \operatorname{dist}\left((x, y), \operatorname{Sg} u_{0 *}\right) \leq \sigma\right\}, & x \in B_{2 R_{0}}(0) \\ u_{0}(x), & \text { otherwise }\end{cases}
$$


with $\sigma>0$. Let $u^{\varepsilon, \sigma}$ be the solution of (7.3) with initial data $u_{0}$. Let $\bar{u}^{\sigma}$ denote $=\limsup \operatorname{sun}_{\varepsilon \rightarrow 0}^{*} u^{\varepsilon, \sigma}$. A symmetric argument to the proof of Theorem 7.3 implies $\underline{u}>\bar{u}^{\sigma}$ in $\mathbf{R}^{n} \times(0, \infty)$. In particular, we have

$$
\underline{u} \geq w:=\sup _{0<\sigma<1} \bar{u}^{\sigma} \quad \text { in } \mathbf{R}^{n} \times(0, \infty) .
$$

Although we do not give the proof, it is possible to prove that the supremum of a family of proper subsolutions is also a proper subsolution; such a property is wellknown for conventional subsolutions [30]. Thus $w^{*}$ is a proper subsolution since $\bar{u}^{\sigma}$ is a proper subsolution by Theorem 6.1. By the contraction principle we see $w=\underline{u}$ a.e. Thus $\underline{u} \leq w^{*}$ everywhere. Since $w^{*} \leq(\underline{u})^{*}$, this implies that $U=(\underline{u})^{*}=w^{*}$. Thus $U$ is a proper subsolution. Since $U_{*}=\underline{u}$ so that $U_{*}$ is a proper supersolution by Theorem $6.1, U$ is a upper semicontinuous proper solution. It is easy to see that the initial condition is attained (by Theorem 6.2) and that $\left(U_{*}\right)^{*}=U$.

(ii) (Uniqueness) Using $\underline{u}_{\delta}$ and $\bar{u}^{\sigma}$ as in the proof of Theorem 7.3 we are able to prove that any proper solution of $(7.1)$ with initial data $u_{0}$ agrees with the entropy solution almost everywhere provided $u_{0}$ satisfies the assumptions of Theorem 7.3 (ii).

(iii) Results similar to Theorem 7.3 and Remark 7.4 (i), (ii) hold for periodic initial data. For example the entropy solution $u$ of (7.1) with initial data $u_{0} \in C\left(\mathbf{T}^{n}\right)$ has a representative $\tilde{u}$ (i.e. $u=\tilde{u}$ a.e.) which is a proper solution of (7.1) with initial data $u_{0}$. What is unclear is the uniqueness of proper solution for general initial data $u_{0} \in C\left(\mathbf{T}^{n}\right)$ although it is unique in almost everywhere sense.

Corollary 7.5. Assume the same hypotheses of Theorem 7.3 (i), (ii) concerning $f^{i}, u_{0}, u^{\varepsilon}$ and $\bar{u}$. Assume that $u_{0}$ is strictly spokewise decreasing. Then sg $u^{\varepsilon}$ converges to sg $\bar{u}$ in the sense of Hausdorff distance topology in $B_{R}(0) \times \mathbf{R} \times[0, T]$ for any $T>0, R>0$. In particular, $u^{\varepsilon}$ converges to $\bar{u}$ locally uniformly outside the jump discontinuities $\Sigma$ of $\bar{u}$. Moreover, $\bar{u}$ is an entropy solution of (7.1) with initial data $u_{0}$.

This follows from Theorems 6.10 and 7.3. We have thus proved Corollary 1.5. We warn the reader that $\underline{u}=\bar{u}$ a.e. does not necessarily imply $(\underline{u})_{*}=\bar{u}$. In general we have proved that $(\underline{u})^{*}=\left((\bar{u})_{*}\right)^{*}$ but this does not imply $(\underline{u})^{*}=\bar{u}$. Unless strictly spokewise monotonicity condition on $u_{0}$ is imposed, the present argument does not yield $(\underline{u})^{*}=\bar{u}$ although it is likely to hold.

Acknowledgements. The author is grateful to Professor Katsuo Tsukamoto who kindly let him know the article [39]. He is also grateful to Professor Jonathan 
Goodman and Professor Zhouping Xin for valuable comments on references related to conservation laws. He is also grateful to Professor Yoshihiro Tonegawa and Professor Shun'ichi Goto for their comments on earlier versions of this paper. He is also grateful to Dr. Mi-Ho Giga for valuable suggestions. This work is partly supported by the Grant-in-Aid for Scientific Research No.10304010, No.12874024, the Japen Society for the Promotion of Science.

\section{References}

[1] S. Angenent and M. Fila, Interior gradient blow-up in a semilinear parabolic equation, Differential and Integral equations, 9(1996), 865-877.

[2] S. B. Angenent and M. E. Gurtin, Multiphase thermomechanics with interfacial structure 2. Evolution of an isothermal interface, Arch. Rational Mech. Anal., 108(1989), 323-391.

[3] M. Bardi and I. Capuzzo-Dolcetta, Optimal Control and Viscosity Solutions of Hamilton-Jacobi-Bellman Equation, Systems \& Control: Foundations \& Applications, Birkhäuser, Boston, (1997).

[4] G. Barles, Solutions de Viscosité des Equations de Hamilton-Jacobi, Mathématiques \& Applications, vol.17, Springer-Verlag, Paris, (1994).

[5] G. Barles, H. M. Soner and P. E. Souganidis, Front propagation and phase field theory, SIAM J. Cont. Opt. 31(1993), 439-469.

[6] E. N. Barron and R. Jensen, Semicontinuous viscosity solutions for HamiltonJacobi equations with convex Hamiltonians, Commun. in Partial Differential Equations, 15(1990), 1713-1742.

[7] E. N. Barron and R. Jensen, Optimal control and semicontinuous viscosity solutions, Proc. AMS, 113(1991), 397-402.

[8] Y.-G. Chen, Y. Giga and S. Goto, Uniqueness and existence of viscosity solutions of generalized mean curvature flow equation, J. Differential Geometry, 33(1991), 749-786. (Announcement: Proc. Japan Acad., Ser.A 65(1989), 207210.)

[9] Y.-G. Chen, Y. Giga and S. Goto, Remarks on viscosity solutions for evolution equations, Proc. Japan Acad., Ser.A 67(1991), 323-328. 
[10] M. G. Crandall, H. Ishii and P.-L. Lions, User's guide to viscosity solutions of second order partial differential equations, Bull. Amer. Math. Soc., 27(1992), $1-67$.

[11] M. G. Crandall and P.-L. Lions, Viscosity solutions of Hamilton-Jacobi equations, Trans. Amer. Math. Soc., 277(1983), 1-42.

[12] C. M. Dafermos, Hyperbolic Conservation Laws in Continuum Physics, Springer (2000), Berlin.

[13] L. C. Evans, The perturbed test function method for viscosity solutions of nonlinear PDE, Proc. Royal Soc. of Edinburgh 111A(1989), 359-375.

[14] L. C. Evans and J. Spruck, Motion of level sets by mean curvature, I, J. Differential Geometry 33(1991), 635-681.

[15] M.-H. Giga and Y. Giga, Evolving graphs by singular weighted curvature, Arch. Rational Mech. Anal. 141(1998), 117-198.

[16] M.-H. Giga and Y. Giga, A subdifferential interpretation of crystalline motion under nonuniform driving force, Proc. of the International Conference in Dynamical Systems and Differential Equations, Springfield Missouri, (1996), "Dynamical Systems and Differential Equations", (eds. W.-X. Chen and S.-C. $\mathrm{Hu}$ ), Southwest Missouri Univ. vol.1(1998), pp.276-287.

[17] M.-H. Giga and Y. Giga, Stability for evolving graphs by nonlocal weighted curvature, Commun. in Partial Differential Equations, 24(1999), 109-184.

[18] M.-H. Giga and Y. Giga, Motion driven by nonlocal curvature in the plane, In: Asymptotics in Nonlinear Diffusive Systems, (eds. Y. Nishiura et al.), Tohoku Math. Publ. 8(1998), 75-83.

[19] M.-H. Giga and Y. Giga, Crystalline and level set flow - Convergence of a crystalline algorithm for a general anisotropic curvature flow in the plane, Gakuto International Series, Math. Sciences and Appl. vol 13(2000) Free Boundary Problems: Theory and Applications (ed. N. Kenmochi), pp.64-79.

[20] M.-H. Giga and Y. Giga, Generalized motion by nonlocal curvature in the plane, Hokkaido University Preprint Series in Math. 478(2000). 
[21] M.-H. Giga, Y. Giga and R. Kobayashi, Very singular diffusion equations, Proc. of Taniguchi Conf. on Mathematics, Nara 98 (ed. T. Sunada), Advances Studies in Pure Mathematics 26 (2000), to appear.

[22] Y. Giga, Interior derivative blow-up for quasilinear parabolic equations, Discrete Cotin. Dynam. Systems, 1(1995), 449-461.

[23] Y. Giga, A level set method for surface evolution equations, Sugaku Expositions, 10(1997), 217-241 (translated from Sūgaku 47(1995), 321-340).

[24] Y. Giga, Shocks and very strong vertical diffusion, Hokkaido University Preprint Series in Math. 510(2001).

[25] Y. Giga, M. Paolini and P. Rybka, On the motion by singular interfacial energy, Japan J. Indust. Appl. Math., to appear.

[26] Y. Giga and M.-H. Sato, On semicontinuous solutions for general HamiltonJacobi equations, Proc. Japan Acad., Ser.A 75(1999), 159-162.

[27] Y. Giga and M.-H. Sato, A level set approach to semicontinuous viscosity soIutions for Cauchy problems, Commun. in Partial Differential Equations, to appear.

[28] J. Goodman, A. Kurganov and P. Rosenau, Breakdown in Burgers-type equations with saturating dissipation fluxes, Nonlinearity, 12(1999), 247-268.

[29] J. Goodman and Z. P. Xin, Viscous limits for piecewise smooth solutions to systems of conservation laws, Arch. Rational Mech. Anal., 121(1992), 235-265.

[30] H. Ishii, Perron's method for Hamiton-Jacobi equations, Duke Math. J., $\mathbf{5 5}(1987)$, 369-384.

[31] B. Kawohl and N. Kutev, Viscosity solutions for degenerate and nonmonotone elliptic equations, In: Applied Nonlinear Analysis (eds. Sequeira et al) (1999), Kluwer Academic/ Plenum Pub., New York pp.231-254.

[32] R. Kobayashi and Y. Giga, Equations with singular diffusivity, J. Stat. Phys. 95(1999), 1187-1220.

[33] S. Kružkov, First order quasilinear equations with several independent variables, Mat. Sbornik 123(1970), 228-255. English translation: Math. USSR Sbornik 10 (1970), 217-273. 
[34] M. M. Lavrentiev, Jr., Gradient blow-up solutions to parabolic problems: Examples and available solvability results, preprint University of Wollongong (2000).

[35] P.-L. Lions, Generalized Solutions of Hamilton-Jacobi equations, Research Notes in Math. 69, Pitman, Boston-London-Melbourne, (1982).

[36] A. Lunardi, Analytic Semigroups and Optimal Regularity in Parabolic Problems, Progress in Nonlinear Differential Equations and their Applications, 16 Birkhäuser, Basel, (1995)

[37] S. Osher and J. A. Sethian, Fronts propagating with curvature - dependent speed: Algorithms based on Hamilton-Jacobi formulations, J. Comput. Phys., $\mathbf{7 9}(1988)$, 12-49.

[38] H. M. Soner, Motion of a set by the curvature of its boundary, J. Differential Equations, 101(1993), 313-372.

[39] I. Sunagawa and P. Bennema, Morphology of growth spirals; Theoretical and Experimental, In: Preparation and Properties of Solid State Materials, vol.7 (ed. W. R. Wilcox) (1982), pp.1-129, Marcel Dekker, New York.

[40] J. Taylor, Constructions and conjectures in crystalline nondifferential geometry, In: Differential Geometry (eds. B. Lawson and K. Tanenblat), Proceedings of the Conference on Differential Geometry, Rio de Janeiro, Pitman Monographs Surveys in Pure and Applied Math. 52(1991) pp.321-336, Pitman, London

[41] S.-H. Yu, Zero-dissipation limit of solutions with shocks for systems of hyperbolic conservation laws, Arch. Rational Mech. Anal., 146(1999), 275-370.

\section{YOSHIKAZU GIGA}

Department of Mathematics

Hokkaido University

Sapporo 060-0810

Japan

E-mail: giga@math.sci.hokudai.ac.jp 